Fengkai Zhang*, Lucian P. Smith, Michael L. Blinov, James Faeder, William S. Hlavacek, Jose Juan Tapia, Sarah M. Keating, Nicolas Rodriguez, Andreas Dräger, Leonard A. Harris, Andrew Finney, Bin Hu, Michael Hucka and Martin Meier-Schellersheim

\title{
Systems biology markup language (SBML) level 3 package: multistate, multicomponent and multicompartment species, version 1, release 2
}

https://doi.org/10.1515/jib-2020-0015

Received April 1, 2020; accepted April 20, 2020; published online July 6, 2020

\begin{abstract}
Rule-based modeling is an approach that permits constructing reaction networks based on the specification of rules for molecular interactions and transformations. These rules can encompass details such as the interacting sub-molecular domains and the states and binding status of the involved components. Conceptually, fine-grained spatial information such as locations can also be provided. Through "wildcards" representing component states, entire families of molecule complexes sharing certain properties can be specified as patterns. This can significantly simplify the definition of models involving species with multiple components, multiple states, and multiple compartments. The systems biology markup language (SBML) Level 3 Multi Package Version 1 extends the SBML Level 3 Version 1 core with the "type" concept in the Species and Compartment classes. Therefore, reaction rules may contain species that can be patterns and exist in multiple locations. Multiple software tools such as Simmune and BioNetGen support this standard that thus also becomes a medium for exchanging rule-based models. This document provides the specification for Release 2 of Version 1 of the SBML Level 3 Multi package. No design changes have been made to the description of models between Release 1 and Release 2; changes are restricted to the correction of errata and the addition of clarifications.
\end{abstract}

Keywords: rule-based modeling; specification; standard; systems biology.

*Corresponding author: Fengkai Zhang, NIAID/NIH, Bethesda, USA, E-mail: zhangfen@nih.gov. https://orcid.org/0000-00017112-9328

Lucian P. Smith: University of Washington, Seattle, USA, E-mail: Ipsmith@uw.edu. https://orcid.org/0000-0001-7002-6386 Michael L. Blinov: UConn Health, Farmington, USA, E-mail: blinov@uchc.edu. https://orcid.org/0000-0002-9363-9705

James Faeder and Jose Juan Tapia: University of Pittsburgh, Pittsburgh, USA, E-mail: faeder@pitt.edu (J. Faeder), jjtapia@gmail.com (J. Juan Tapia). https://orcid.org/0000-0001-8127-609X (J. Faeder) . https://orcid.org/0000-0002-8392-6022 (J. Juan Tapia)

William S. Hlavacek and Bin Hu: Los Alamos National Laboratory, Los Alamos, USA, E-mail: wish@lanl.gov (W.S. Hlavacek), bhu@lanl.gov (B. Hu). https://orcid.org/0000-0003-4383-8711 (W.S. Hlavacek) . https://orcid.org/0000-0002-3775-387X (B. Hu) Sarah M. Keating: European Bioinformatics Institute, Cambridge, UK, E-mail: keating.sm@googlemail.com. https://orcid.org/ 0000-0002-3356-3542

Nicolas Rodriguez: Babraham Institute, Cambridge, UK, E-mail: nicolas.rodriguez@babraham.ac.uk. https://orcid.org/00000002-9290-7894

Andreas Dräger: Computational Systems Biology of Infection and Antimicrobial-Resistant Pathogens, Institute for Bioinformatics and Medical Informatics (IBMI), University of Tübingen, Tübingen, Germany, E-mail: andreas.draeger@uni-tuebingen.de. https:// orcid.org/0000-0002-1240-5553

Leonard A. Harris: Vanderbilt University School of Medicine, Nashville, USA, E-mail: l.harris@vanderbilt.edu. https://orcid.org/ 0000-0003-2112-6940

Andrew Finney: Ansys UK Ltd, Abingdon, UK; Department of Computer Science, University of Tübingen, Tübingen, Germany; and German Center for Infection Research (DZIF), partner site Tübingen, Germany, E-mail: andrewmartinfinney@gmail.com Michael Hucka: California Institute of Technology, Pasadena, USA, E-mail: mhucka@caltech.edu. https://orcid.org/0000-0001-9105-5960 Martin Meier-Schellersheim: NIAID/NIH, Bethesda, USA, E-mail: mms@niaid.nih.gov. https://orcid.org/0000-0002-8754-6377 
SBML Level 3 Package Specification

\section{Multistate, Multicomponent and Multicompartment Species Package for SBML Level 3}

\author{
Fengkai Zhang \\ zhangfen@niaid.nih.gov \\ NIAID/NIH \\ USA
}

\author{
Martin Meier-Schellersheim \\ mms@niaid.nih.gov \\ NIAID/NIH \\ USA
}

Version 1, Release 2

17 April 2020

The latest release, past releases, and other materials related to this specification are available at http://sbml.org/Documents/Specifications/SBML_Level_3/Packages/multi

This release of the specification is available at

http://identifiers.org/combine.specifications/sbml.level-3.version-1.multi.version-1.release-2

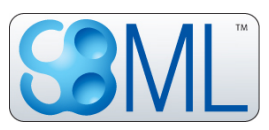




\section{Contributors}

\begin{tabular}{|c|c|}
\hline Bastian R. Angermann & Frank Bergmann \\
\hline NIAID/NIH & California Institute of Technology \\
\hline USA & USA \\
\hline Michael L. Blinov & Andreas Dräger \\
\hline UConn Health & Universtiy of Tuebingen \\
\hline USA & Germany \\
\hline James Faeder & Andrew Finney \\
\hline University of Pittsburgh School of Medicine & University Hertfordshire \\
\hline USA & United Kingdom \\
\hline Leonard A. Harris & William S. Hlavacek \\
\hline University of Pittsburgh School of Medicine & Los Alamos National Laboratory \\
\hline USA & USA \\
\hline Stefan Hoops & Bin $\mathrm{Hu}$ \\
\hline Virginia Tech & Los Alamos National Laboratory \\
\hline USA & USA \\
\hline Michael Hucka & Sarah M. Keating \\
\hline California Institute of Technology & European Bioinformatics Institute \\
\hline USA & United Kingdom \\
\hline Nicolas Le Novère & Anika Oellrich \\
\hline Babraham Institute & European Bioinformatics Institute \\
\hline United Kingdom & United Kingdom \\
\hline Nicolas Rodriguez & Lucian P. Smith \\
\hline European Bioinformatics Institute & University of Washington \\
\hline United Kingdom & USA \\
\hline \multicolumn{2}{|l|}{ Jose Juan Tapia } \\
\hline $\begin{array}{l}\text { University of Pittsburgh School of Medicine } \\
\text { USA }\end{array}$ & \\
\hline
\end{tabular}

And all the people who contributed to the discussions on the sbml-multi mailing list. 


\section{Contents}

1 Introduction $\mathbf{5}$

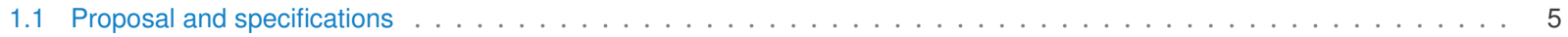

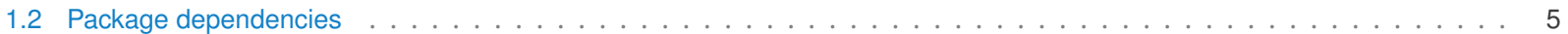

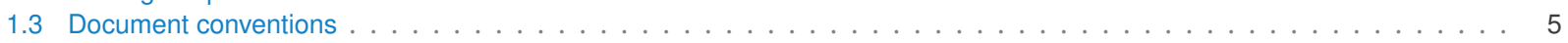

2 Background and context 6

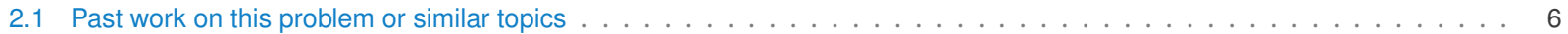

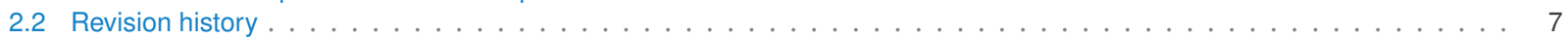

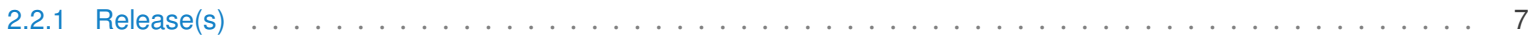

2.2 .2 Release Candidates . . . . . . . . . . . . . . . . . . . . . . . . . . . 7

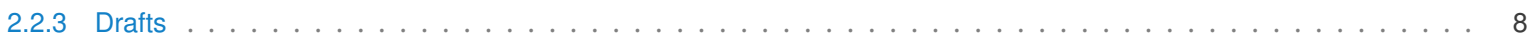

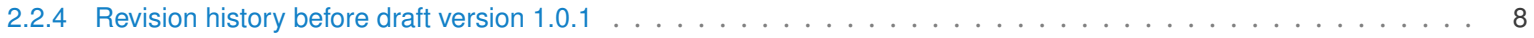

3 Package syntax and semantics 9

3.1 Namespace URI and other declarations necessary for using this package . . . . . . . . . . . . . . . 9

3.2 Primitive data types . . . . . . . . . . . . . . . . . . . . . . . . . . . . . 9

3.2 .1 Type Bindingstatus . . . . . . . . . . . . . . . . . . . . . . . . . . . 9

3.2 .2 Type Relation . . . . . . . . . . . . . . . . . . . . . . . . . . . . . . 9

3.2.3 Type RepresentationType . . . . . . . . . . . . . . . . . . . . . . . . . . . . . 10

3.3 The new and extended classes in the Multi Package . . . . . . . . . . . . . . . . . . . . . 10

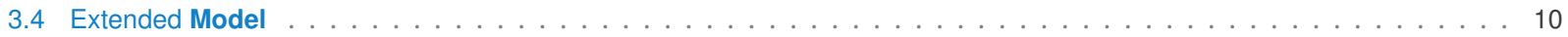

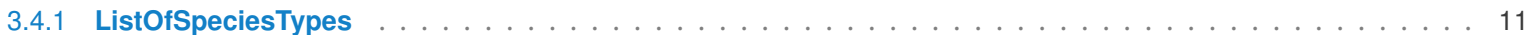

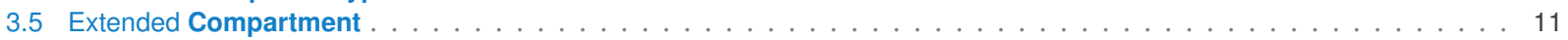

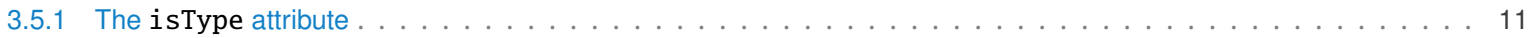

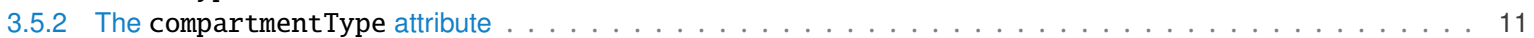

3.5 .3 ListOfCompartmentReferences . . . . . . . . . . . . . . . . . . . . . . . . 12

3.6 CompartmentReference . . . . . . . . . . . . . . . . . . . . . . . . . . . . . . 12

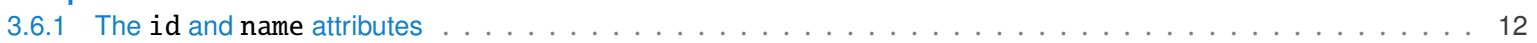

3.6 .2 The compartment attribute . . . . . . . . . . . . . . . . . . . . . . . . . . . 12

3.7 The relationship of Compartment, CompartmentReference and ListOfCompartmentReferences . . . . . . . . . . 12

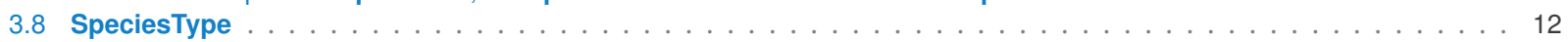

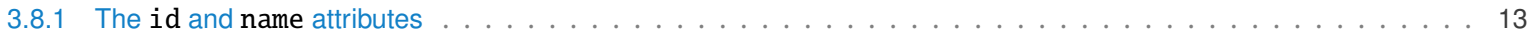

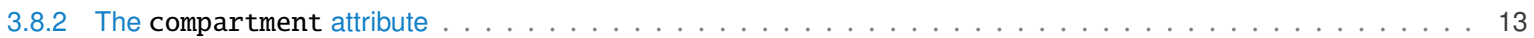

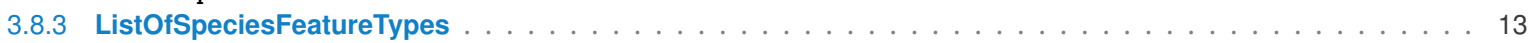

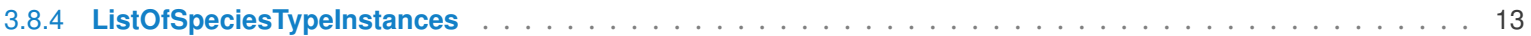

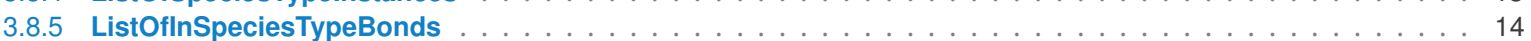

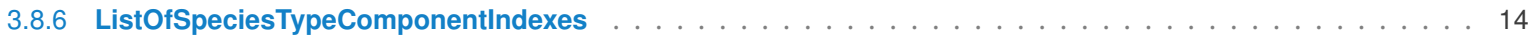

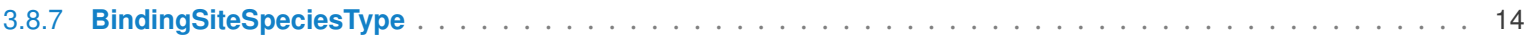

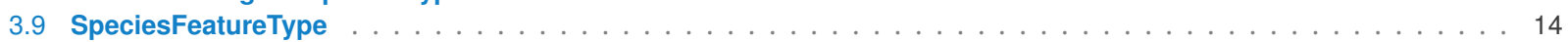

3.9 .1 The $\mathrm{id}$ and name attributes . . . . . . . . . . . . . . . . . . . . . . . . . . . . . . . .

3.9 .2 The occur attribute . . . . . . . . . . . . . . . . . . . . . . . . . . . . . . . 14

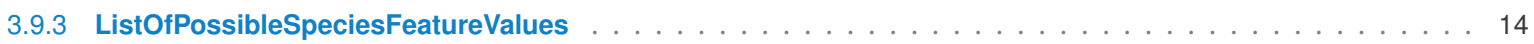

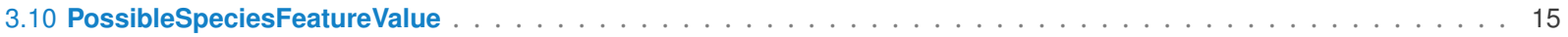

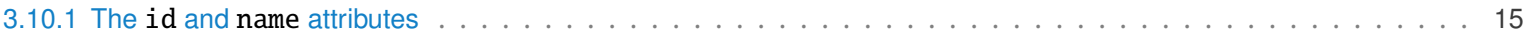

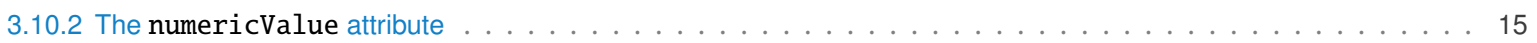

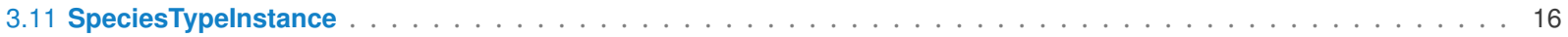

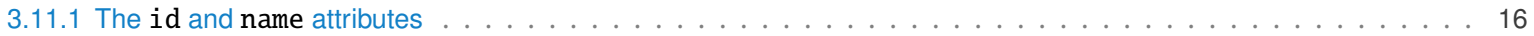

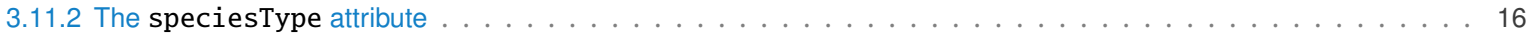

3.11 .3 The compartmentReference attribute . . . . . . . . . . . . . . . . . . . . . 16

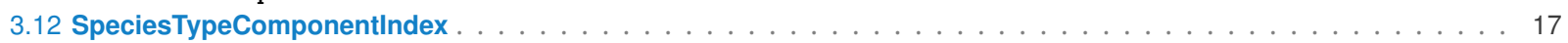

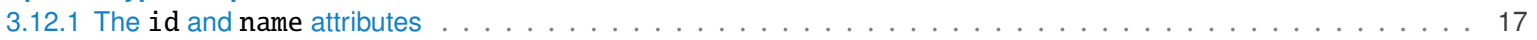

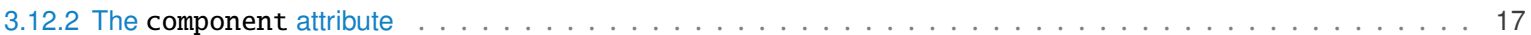

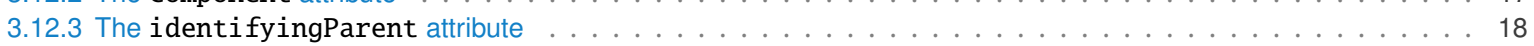

3.12 .4 Reference a component in a speciesType or a species . . . . . . . . . . . . . . . 18

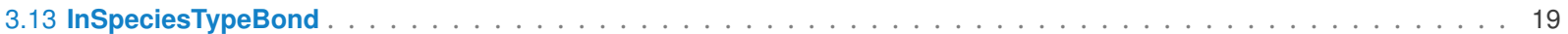

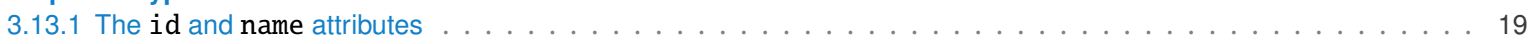

3.13 .2 The bindingSite 1 and bindingSite 2 attributes . . . . . . . . . . . . . . . . 20

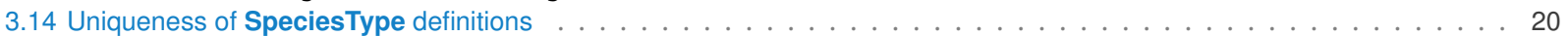

3.15 Extended Species . . . . . . . . . . . . . . . . . . . . . . . . . . . . . . . . . 22

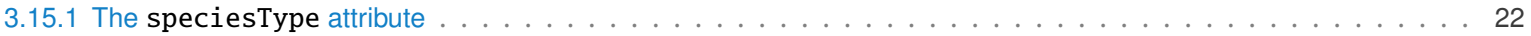

3.15 .2 ListOfOutwardBindingSites . . . . . . . . . . . . . . . . . . . . . . . . 22

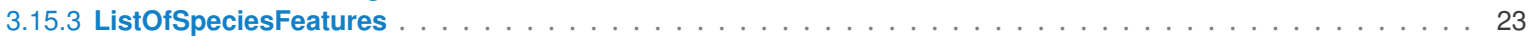

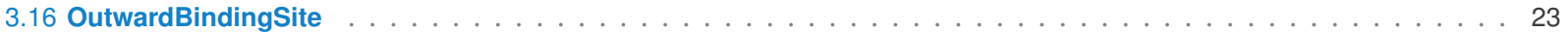




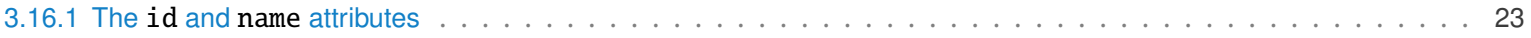

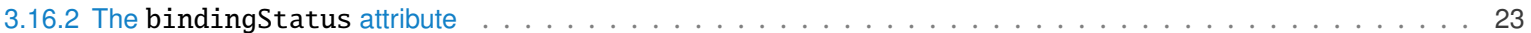

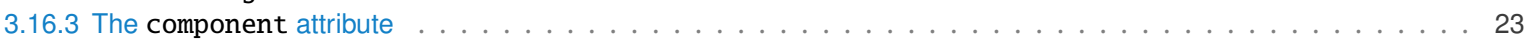

3.16 .4 Example . . . . . . . . . . . . . . . . . . . . . . . . . . . . . . . . . . . 24

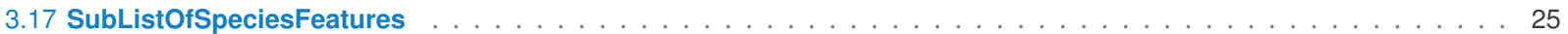

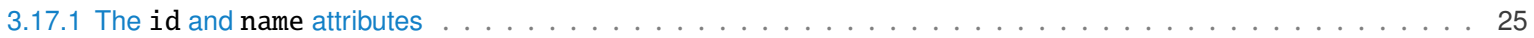

3.17 .2 The relation attribute . . . . . . . . . . . . . . . . . . . . . . . . . 25

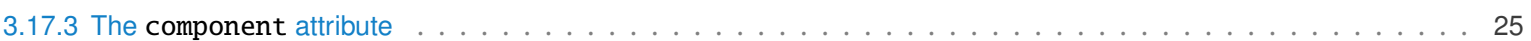

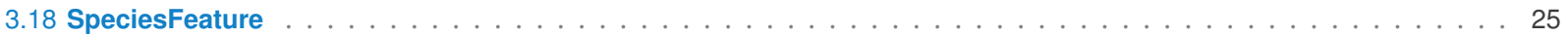

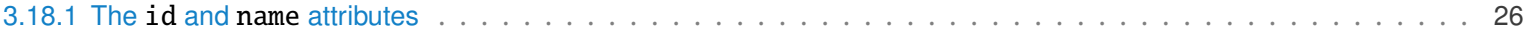

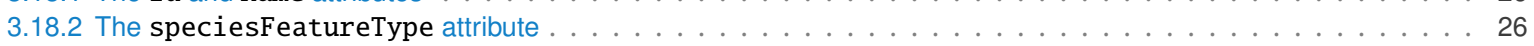

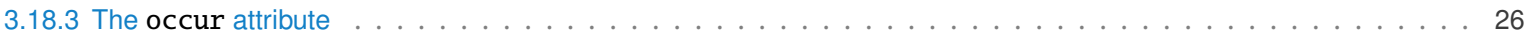

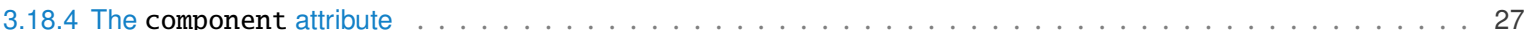

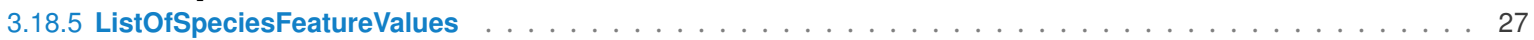

3.18 .6 SpeciesFeatureValue . . . . . . . . . . . . . . . . . . . . . . . . . . . . . . . . 27

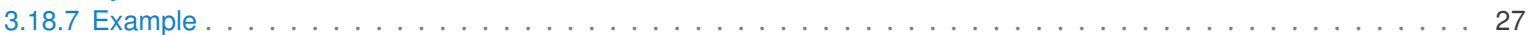

3.19 Fully defined species and mapping to pattern species . . . . . . . . . . . . . . . . . . . . . . 29

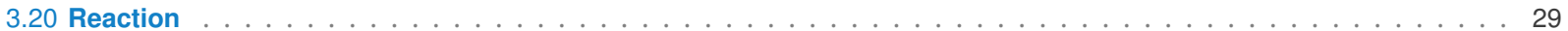

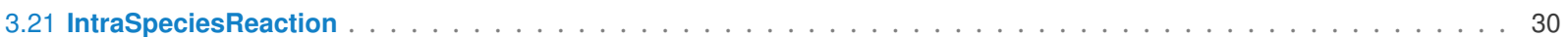

3.22 Extended SimpleSpeciesReference . . . . . . . . . . . . . . . . . . . . . . . . . 30

3.23 Extended SpeciesReference . . . . . . . . . . . . . . . . . . . . . . . . . . . . . . . . . . 32

3.23.1 ListOfSpeciesTypeComponentMapsInProduct . . . . . . . . . . . . . . . . . . . . . . . . . 32

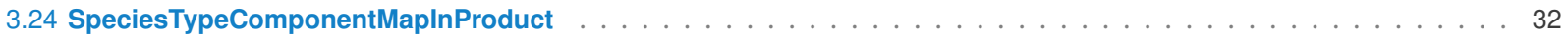

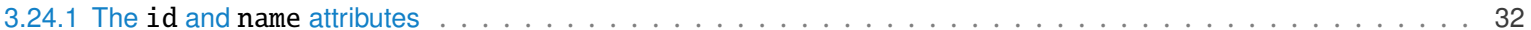

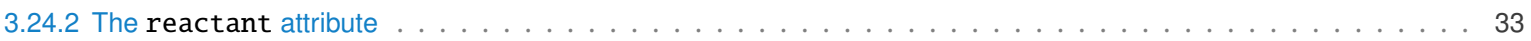

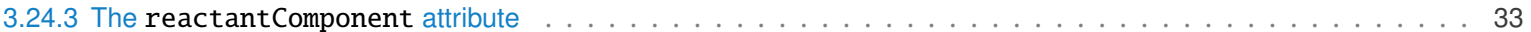

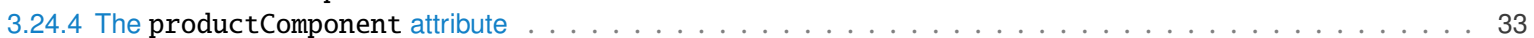

3.25 OutwardBindingSites and speciesFeatures in don't care state in reaction products . . . . . . . . . . . . 33

3.26 Extended ci elements in Math objects . . . . . . . . . . . . . . . . . . . . . . . . . . . 33

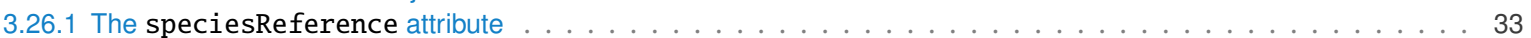

3.26 .2 The representationType attribute . . . . . . . . . . . . . . . . . . . . . . . 34

3.27 Namespace scoping rules for identifiers . . . . . . . . . . . . . . . . . . . . . . . . . . 35

4 Examples 36

4.1 Example: Compartment, SpeciesType, and Species . . . . . . . . . . . . . . . . . . . . . 36

4.2 Simmune example: the Ecad model . . . . . . . . . . . . . . . . . . . . . . . . . . . . . . 37

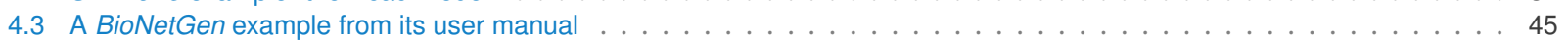

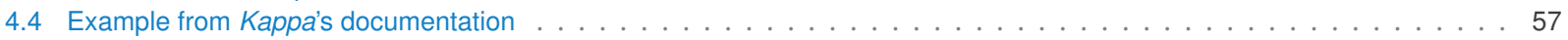

A Validation of SBML documents using Multi constructs 60

Acknowledgments

References

$\begin{array}{lll}\text { Section Contents } & \text { Page } 4 \text { of } 74\end{array}$ 


\section{Introduction}

This Multistate, Multicomponent and Multicompartment Species (Multi) package provides an extension of SBML Level 3 [Hucka et al. (2016)] that supports encoding models with molecular complexes that have multiple components and can exist in multiple states and in multiple compartments. One of its goals is to provide a platform for sharing models based on the specifications of molecular transformations/interactions and the rules governing such reactions [Angermann et al. (2012); Feret et al. (2009); Hlavacek et al. (2006); Zhang et al. (2013)]. This specification covers the goals and features described in the previous Multi proposal [Le Novère and Oellrich (2010)] for extending SBML to carry the information for multistate multicomponent species with revised data structure. In addition, this specification includes the feature for multicompartment species as described in the releases of the Multi proposal [Zhang and Meier-Schellersheim (2013a), Zhang et al. (2012)].

\subsection{Proposal and specifications}

The proposal corresponding to this package specification is available at:

http://sbml.org/Community/Wiki/SBML_Level_3_Proposals/Multistate_and_Multicomponent_Species_ Proposal

The specifications (v1.0.1 to current) are located at:

https://sourceforge.net/p/sbml/code/HEAD/tree/trunk/specifications/sbml-1evel-3/version-1/ multi/spec/

\subsection{Package dependencies}

The Multi package has no dependencies on other SBML Level 3 packages.

\subsection{Document conventions}

UML 1.0 notation is used in this document to define the constructs provided by this package. Colors in the diagrams carry the following additional information for the benefit of those viewing the document on media that can display color:

- Black Items colored black are components taken unchanged from their definitions in the SBML Level 3 Core specification document.

Class boxes are also drawn with with dashed lines to further distinguish them.

- Blue Items colored blue are new components introduced in this package specification. They have no equivalent in the SBML Level 3 Core specification.

- Red

For other matters involving the use of UML, XML and typographical conventions, this document follows the conventions used in the SBML Level 3 Core specification document [Hucka et al. (2016)].

For simplicity, "..." in all example code refers to some unspecified code content, that is not important for the purpose of illustrating the issue at hand.

We use red color in text to indicate changes between this version of the specification, namely SBML Multi Package Version 1 Release 2, and the most recent previous release of the specification (which, for the present case, is SBML Multi Package Version 1 Release 1). 


\section{Background and context}

Rule-based, domain-detailed modeling has been extremely valuable in systems biology related studies [Manes et al. (2015) and Miskov-Zivanov1 et al. (2013)]. Rule-based, domain-detailed modeling approaches (BioNetGen [Faeder et al. (2009)], Kappa [Danos and Laneve (2004)], and Simmune [Angermann et al. (2012); Meier-Schellersheim et al. (2006)]) define rules for transformations of molecular domains and interactions between pairs of molecule domains, specifying how the transformations/interactions depend on particular states of the molecules (pattern) and their locations in specific compartments. In order to generate networks of biochemical reactions these rules are applied to the molecular components of the systems to be modeled, either at the beginning of the modeling (simulation) process or "on the fly" (as molecule complexes emerge from the interaction rules). Expressing such rule-based, domain-detailed reaction networks using the concepts of Species and Compartment in SBML (L3 core and L2) can be difficult for rules and molecule sets that lead to large numbers of resulting molecular complexes. It would therefore be desirable to have an SBML standard for encoding rule-based, domain-detailed models using their "native" concepts for describing reactions instead of having to apply the rules and unfold the networks prior to encoding in an SBML format.

We proposed a revised proposal of the Multi package: "Multistate, Multicomponent and Multicompartment Species Package for SBML Level 3" (abbreviated as Multi) [Zhang et al. (2012) and Zhang and Meier-Schellersheim (2013a)] which takes the scopes and some data structures developed in the previous Multi proposal [Le Novère and Oellrich (2010)] and addresses main issues arising from a rule-based, domain-detailed modeling point of view with the data structures consistent with that used in the available rule-based, domain-detailed modeling tools.

Note:

This specification was developed with the main goal of taking into account molecular transformations and bimolecular interactions mediated through specific binding domains (or sites). Models without such detailed description of the molecular interactions can be encoded as well if the other features in this specification such as SpeciesFeatureType, SpeciesFeature, and extended Compartment satisfy the model requirements.

\subsection{Past work on this problem or similar topics}

- Nicolas Le Novère and Anika Oellrich proposed the previous version of the Multi proposal [Le Novère and Oellrich (2010)]. However, it was realized that a more detailed treatment of molecular binding sites and their state-dependent interactions would be desirable.

- In August 2012, Fengkai Zhang from the Simmune group presented " Draft for discussion SBML Proposals for Revised Multi, Simple Spatial and Multi-Spatial Extensions" at COMBINE 2012 [Zhang et al. (2012)]. The three proposals cover the goals and scope of the previous Multi proposal (2010), revise it and add some new features that improve usage of the proposal for rule-based approaches.

- Based on the discussions and suggestions received during COMBINE 2012 as well as on feedback from the SBML discussion forum, the new Multi proposal [Rev 221, Zhang and Meier-Schellersheim (2012)] was released to the SBML-Multi community, which integrates and covers most of the features in the three previous proposals of August 2012.

- In May 2013, a new revision (rev 280) of the Multi proposal [Zhang and Meier-Schellersheim (2013a)] was released before the meeting of HARMONY 2013. The extended Compartment class and its related classes have been reorganized. All optional boolean attributes have been removed/replaced. A new optional Multi attribute, "whichValue", was added to the ci elements in KineticLaw to identify the sources of species. (Lucian Smith gave many comments/suggestions about this proposal and William Hlavacek gave thoughtful feedback about the BioNetGen example in this proposal). This revision (rev 280) was presented at HARMONY 2013 [Zhang and Meier-Schellersheim (2013b)] with new features to configure multiple occurrences of SpeciesFeatureType. Several new or revised features were discussed during and after HARMONY 2013, including multiple occurrences of SpeciesFeatureType, multiple copies of SpeciesTypelnstance, the numericValue attribute for 
PossibleSpeciesFeatureValue and concentration summation of pattern species. These features are covered or updated in the specifications from v1.0.1.

\subsection{Revision history}

The versioning convention used in this document:

$\mathrm{x} \cdot \mathrm{y} \cdot \mathrm{z}$ (status)

$\mathrm{x}$ : version of SBML Level 3 core.

$y$ : version of the Multi package.

z: release of the Multi package at its version y.

status: "draft", "release candidate", or "release". Absence of status means "release".

For example, the current version is "1.1.2"

$\mathrm{x}=$ " 1 "

$\mathrm{y}=$ " $1 "$

$\mathrm{z}=$ "2"

The followings are the revision history of the Multi package:

\subsubsection{Release(s)}

Version: 1.1.2, this version

- Fix issues raised by the community after "Version 1 release 1".

- Revise parts of the text.

- Add a paragraph to clarify "XML Namespace use" (see Section 3.1 on page 9).

- Validate the complete models and add "demonstration only" notes to those "incomplete" models in the example section (see Section 4 on page 36).

" Version: 1.1.1, March 2017

\subsubsection{Release Candidates}

- Version: 1.1.rc5 (release candidate), March 2017

- Add two validation rules multi-21213 and multi-21214 to check the speciesType attribute of a species with list0fOutwardBindingSites and/or list0fSpeciesFeatures(See Section 3.14 on page 22.)

- Add a constraint to the relation attribute of a subList0fSpeciesFeatures having a speciesFeature child referencing a speciesFeatureType with "occur > 1". (See Section 3.17.2 on page 25 and Section A on page 68.)

- Enforce the SubListOfSpeciesFeatures class to have at least two speciesFeatures and set relation as a required attribute. (See Section 3.17 on page 25.)

- Version: 1.1.rc4 (release candidate), March 2017

More updates on validation rule numbers, line breaks, and the example about SubListOfSpeciesFeatures.

- Version: 1.1.rc3 (release candidate), Febrary 2017

Modify the numbers of several rules to be consistent with the general SBML validation rule conventions.

- Version: 1.1.rc2 (release candidate), January 2017

Add a new validation rule multi-22006 to prevent circular referencing among the extended Compartment objects.

Revise the specification text with minor changes towards a version of the official release candidate. 
- Version: 1.1.rc1 (release candidate), November 2016

Revise the specification text with minor changes towards a version of the official release candidate.

\subsubsection{Drafts}

- Version: 1.0.7 (draft), August 2016

Remove the SpeciesFeatureChange and ListOfSpeciesFeatureChanges classes under SpeciesTypeComponentMapInProduct. The relations expressed in SpeciesFeatureChange can be inferred from the speciesTypeComponentMapInProduct and the species of the mapped reactant and product.

Add a new validation rule 21306, "an outwardBindingSite cannot be a binding site in a bond of the species" (see Section 3.16.3 on page 23 and Section A on page 69)

- Version: 1.0.6 (draft), March 2016

Remove recursively referencing relationship in the ListOfSpeciesFeatures class and add a SubListOfSpeciesFeatures class. See the details in Species.

Version 1.0.6.1 with minor document update is released in April 2016.

- Version 1.0.5 (draft), November 2015

This version has been developed from the previous release v1.0.4 with the following modifications based on the discussion during and after COMBINE 2015 [Zhang (2015)]:

- Drop the occur attribute in the class of SpeciesTypelnstance.

- Drop the occur attribute in the class of SpeciesTypeComponentIndex.

- Drop the class of DenotedSpeciesTypeComponentIndex.

- Revise the scope of PossibleSpeciesFeatureValue ids to be global.

Version 1.0.5.1 with minor document update is released in Dec 2015.

- Version 1.0.4 (draft), June 2015

This version has been developed from the previous release v1.0.3 with minor document update and complete validation rules.

- Version 1.0.3 (draft), April 2015

This version has been developed from the previous release v1.0.2 mainly based on the discussion in COMBINE 2014 with focus on how to facilitate tools to export and import models encoded in the Multi format [Zhang and Meier-Schellersheim (2014)]

- Version 1.0.2 (draft), November 2014

This version has been developed from the previous release v1.0.1 with the following modifications:

- A new BindingSiteSpeciesType sub-class inheriting the SpeciesType class for binding sites. Accordingly, the isBindingSi te attribute has been dropped from SpeciesType.

- Restriction on binding sites which have to be atomic.

- Restriction on SpeciesType that a speciesType cannot have a list0fSpeciesFeatureTypes if it has a list0fInSpeciesTypeBonds.

- A new IntraSpeciesReaction sub-class inheriting the Reaction class for the reactions happening within a Species object. Accordingly, the isIntraSpeciesReaction attribute has been dropped from Reaction.

- Validation rules.

- Version 1.0.1 (draft), September 2013

This was released and presented in COMBINE 2013 [Zhang and Meier-Schellersheim (2013c)], mainly addressing the scenario of multiple occurrences of identical components and/or identical features.

\subsubsection{Revision history before draft version 1.0.1}




\section{Package syntax and semantics}

This section contains a definition of the syntax and semantics of the Multi package for SBML Level 3 Core.

\subsection{Namespace URI and other declarations necessary for using this package}

The following is the namespace URI for this version of the Multi package for SBML Level 3 Core:

"http://www.sbml.org/sbml/level3/version1/multi/version1"

In addition, SBML documents using a given package must indicate whether the package can be used to change the mathematical interpretation of a model. This is done using the attribute required on the $<$ sbml $>$ element in the SBML document. For the Multi package, the value of this attribute must be "true".

The following fragment illustrates the beginning of a typical SBML model using SBML Level 3 Core and this version of the Multi package:

$<$ ?xml version="1.0" encoding="UTF-8"?>

<sbml xmlns="http://www.sbml.org/sbml/level3/version1/core" level="3" version="1"

xmlns:multi="http://www. sbml.org/sbml/level3/version1/multi/version1" multi:required="true">

\section{XML Namespace use}

For element names, XML has clear rules about how to declare and use namespaces. In typical SBML documents, the Multi package namespace will be defined as above, and the Multi package elements will therefore need to be prefixed with "multi:".

In contrast to element names, XML attribute names are completely defined by the element in which they appear, and never have a 'default' namespace defined: the element itself declares whether any attributes should be defined with a namespace prefix or not.

Any attribute that appears in a UML diagram in this specification may either be defined with no namespace prefix, or be defined with the multi namespace as a prefix. For attributes added to SBML core elements (Compartment, Species, SimpleSpeciesReference, and within MathML), the multi namespace prefix is required.

If the Multi package is used in an SBML Level 3 Version 2 document, any element defined here may have either an id or a name with no prefix, as id and name were added to SBase in that level/version.

\subsection{Primitive data types}

The Multi package uses a number of the primitive data types described in section 3.1 of the SBML Level 3 Core Release 2[Hucka et al. (2016)] specification such as SId, SIdRef, string, boolean, int and positiveInteger, and adds three additional primitive types described below.

\subsubsection{Type BindingStatus}

The BindingStatus primitive data type is used in the definition of the OutwardBindingSite class. BindingStatus is derived from type string and its values are restricted to be one of the following possibilities: "bound", "unbound", and "either". Attributes of type BindingStatus cannot take on any other values. The meaning of these three values is discussed in the context of the OutwardBindingSite class in Section 3.16 on page 23.

\subsubsection{Type Relation}

The Relation primitive data type is used in the definition of the SubListOfSpeciesFeatures class. Relation is derived from type string and its values are restricted to be one of the following possibilities: "and", "or", and "not". Attributes of type Relation cannot take on any other values. The meaning of these three values is discussed in the 
context of the SubListOfSpeciesFeatures class in Section 3.17 on page 25.

\subsubsection{Type RepresentationType}

The RepresentationType primitive data type is used in the extension of the ci element.

RepresentationType is derived from type string and its values are restricted to be one of the following possibilities: "sum" or "numericValue". If present, attributes of type RepresentationType cannot take on any other values. The meaning of these values is discussed in the context of the ci element in Section 3.26 on page 33.

\subsection{The new and extended classes in the Multi Package}

The Multi package extends the following object classes:

Model, Compartment, Species, Reaction, SimpleSpeciesReference, and SpeciesReference.

The Multi package defines the following object classes:

CompartmentReference, SpeciesType, SpeciesFeatureType, PossibleSpeciesFeatureValue, SpeciesTypelnstance, InSpeciesTypeBond, SpeciesTypeComponentIndex, SubListOfSpeciesFeatures, OutwardBindingSite,

SpeciesFeature, SpeciesFeatureValue, SpeciesTypeComponentMapInProduct

and "ListOf-" classes:

ListOfSpeciesTypes, ListOfCompartmentReferences, ListOfSpeciesTypelnstances, ListOfSpeciesFeatureTypes, ListOfInSpeciesTypeBonds, ListOfSpeciesTypeComponentIndexes, ListOfPossibleSpeciesFeatureValues, ListOfOutwardBindingSites, ListOfSpeciesFeatures, ListOfSpeciesFeatureValues, ListOfSpeciesTypeComponentMapsInProduct.

All the classes in the Multi package are directly or indirectly derived from SBase, and SBase provides the ability to attach SBO terms as well as MIRIAM annotations. The semantics of a given class in the Multi package can be made more precise by referencing to external controlled vocabularies and ontologies.

Like the classes in SBML Level 3 Core, most new Multi classes have the attribute id (typically mandatory but not all, and of type SId), which serves as an identifier to provide a way to identify the class object. The identifier of a class object reference may or may not carry mathematical interpretation or be used in mathematical formulas, depending on its class and the class object referencing it. The scope of $i d s$ is described in the section of "Namespace scoping rules for identifiers"(Section 3.27 on page 35).

\subsection{Extended Model}

The Multi package extends the Model class of SBML Level 3 Core and adds an optional ListOfSpeciesTypes child to Model. Figure 1 provides the UML diagram for the extended Model class.

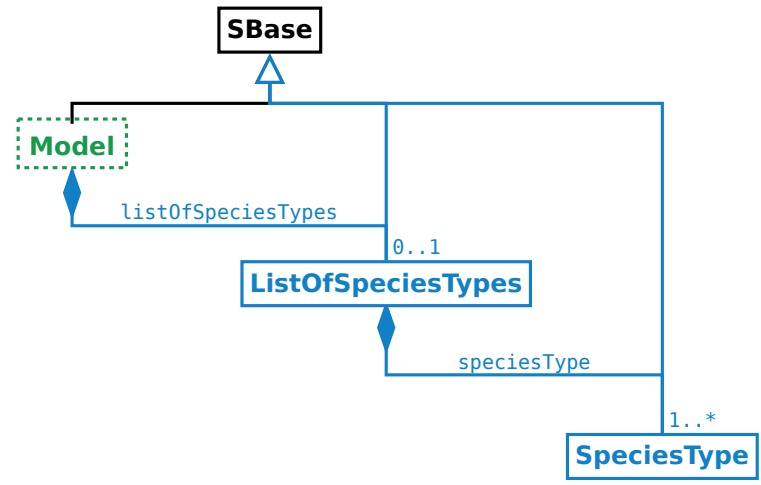

Figure 1: The extension of the Model class. 


\subsubsection{ListOfSpeciesTypes}

ListOfSpeciesTypes is defined in Figure 1 on the preceding page. If present, a ListOfSpeciesTypes object must contain at least one SpeciesType object. Since ListOfSpeciesTypes is derived from SBase, it inherits the sboTerm and metaid attributes, as well as the optional child Notes and Annotation objects.

\subsection{Extended Compartment}

A Compartment object in SBML Level 3 Core represents a bounded space in which species are located. In the Multi package, Compartment is extended. A Multi compartment can be a type that multiple referencing compartments can map to. A Multi compartment can also be a composite compartment or a container that includes other compartments.

The extension of Compartment is defined in Figure 2. The extended Compartment class has a new required attribute isType, a new optional attribute compartmentType and an optional ListOfCompartmentReferences child. The example at Section 4.1 on page 36 illustrates the use of the extended Compartment class.

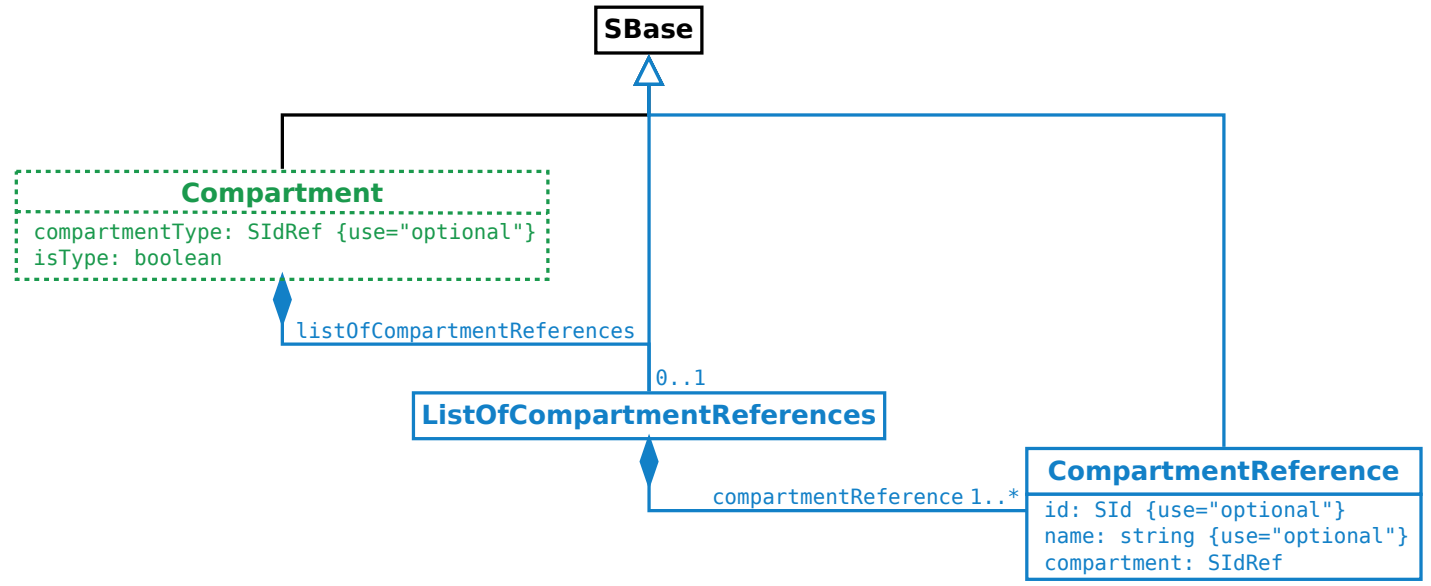

Figure 2: The definitions of Compartment, ListOfCompartmentReferences and CompartmentReference

\subsubsection{The isType attribute}

The required attribute isType, of type boolean, on the Compartment class serves to provide a way to indicate whether the Compartment object is a compartment type.

A Compartment object is a type of compartment if the value of its isType attribute is "true". A type of compartment is a template (in the sense of prototype) for all Compartment objects referencing it (via compartmentType attributes).

Note:

A Species object directly referencing a compartment type is not a fully defined species (see Section 3.19 on page 29).

If the value of the isType attribute is "false", the Compartment object is a "not-a-type" compartment, and it is similar to a SBML core compartment except it can reference a compartment type and can have a ListOfCompartmentReferences child.

\subsubsection{The compartmentType attribute}

The optional attribute compartmentType, of type SIdRef, is used for a "not-a-type" compartment to reference a compartment type. A compartment with the "true" value of its isType attribute cannot have the compartmentType attribute defined. 


\subsubsection{ListOfCompartmentReferences}

ListOfCompartmentReferences is defined in Figure 2 on the preceding page, and is extended from the ListOf class. A list0fCompartmentReferences must have one or more CompartmentReference children. Since ListOfCompartmentReferences is derived from SBase through ListOf, it inherits the sboTerm and metaid attributes, as well as the optional child Notes and Annotation objects.

\subsection{CompartmentReference}

CompartmentReference is defined in Figure 2 on the previous page. It has two optional attributes id and name, and a required attribute compartment. Since CompartmentReference is derived from SBase, it inherits the sboTerm and metaid attributes, as well as the optional child Notes and Annotation objects.

\subsubsection{The id and name attributes}

The optional id attribute, of type SId, serves to provide a way to identify a compartmentReference. CompartmentReference also has an optional name attribute of type string.

Note:

If some or all compartmentReference s within a ListOfCompartmentReferences object reference the same compartment, those compartmentReferences are required to have their id attributes defined to distinguish them.

\subsubsection{The compartment attribute}

The required compartment attribute, of type SIdRef, serves to provide a way to reference a Compartment object.

Note:

A compartmentReference cannot reference a compartment that directly or indirectly contains the compartmentReference. In other words, circular references are not allowed when constructing compartment $s$ and compartmentReferences.

\subsection{The relationship of Compartment, CompartmentReference and ListOfCom- partmentReferences}

In a ListOfCompartmentReferences object, every child compartmentReferences must exclusively reference, directly or indirectly, "not-a-type" compartment which can be of the same compartment type. See the extended Compartment objects in the example in Section 4.1 on page 36.

All compartments referenced by a list0fCompartmentReferences must have the values of their isType attributes the same as that in the parent compartment of the list0fCompartmentReferences. For example, a compartment "A" with isType="true" has a list0fCompartmentReferences referencing two compartments "A1" and "A2". Then, "A1" and "A2" must have isType="true".

\subsection{SpeciesType}

SpeciesType is defined in Figure 3 on the next page and serves to provide backbone structures for species. SpeciesType has one required attribute, id, two optional attributes, name and compartment and four optional ListOf__ objects, including ListOfSpeciesFeatureTypes, ListOfSpeciesTypelnstances, ListOfInSpeciesTypeBonds and ListOfSpeciesTypeComponentIndexes. Since SpeciesType is derived from SBase, it inherits the sboTerm and metaid attributes, as well as the optional child Notes and Annotation objects.

The ListOfSpeciesTypelnstances element provides a way to define multicomponents which are instances of other SpeciesType objects.

The ListOfSpeciesFeatureTypes element and its SpeciesFeatureType child set up a framework for the referencing species or the instances of speciesTypes to be able to have multistates. The ListOfSpeciesTypeComponent- 
Indexes element provides a way to reference a component in a speciesType.

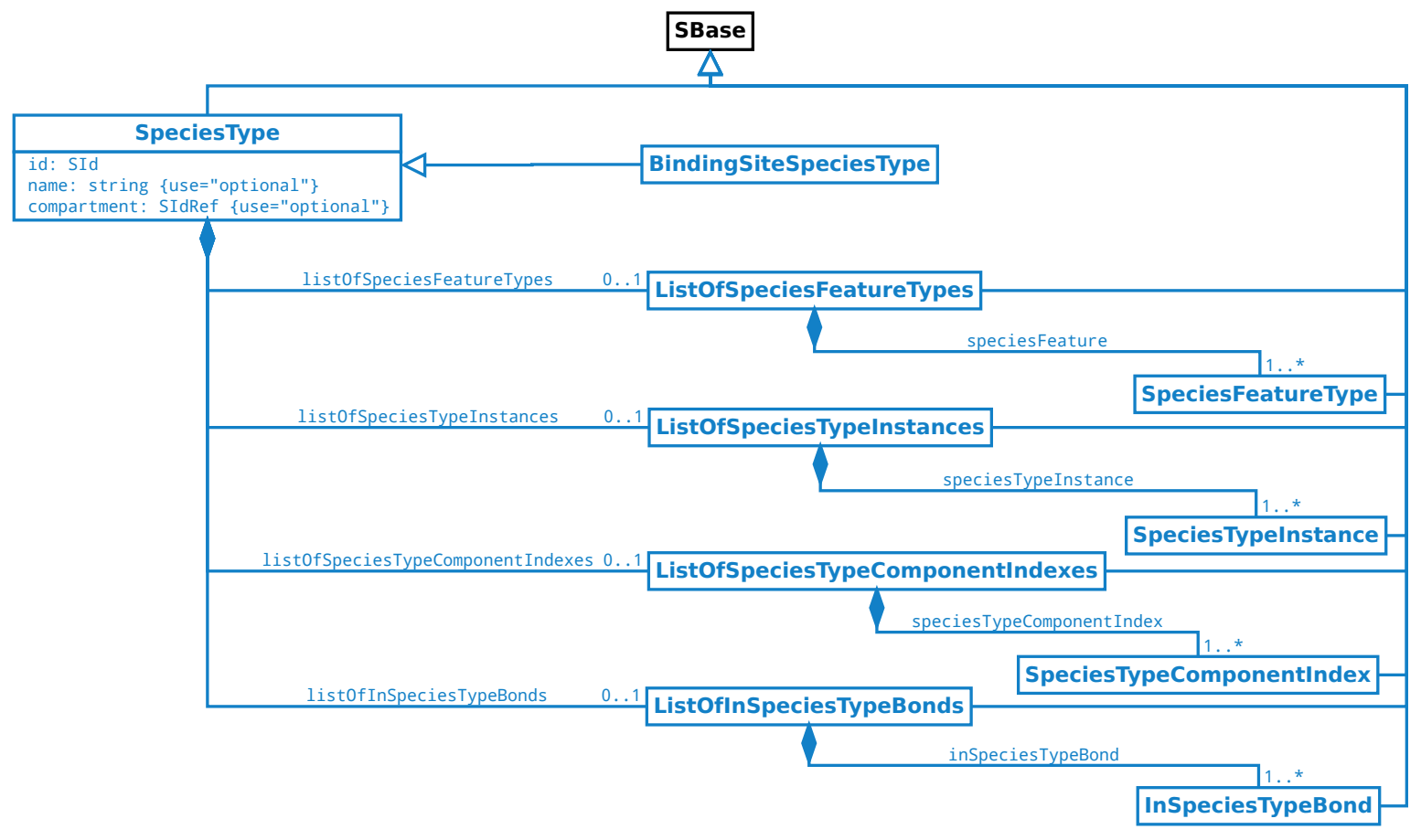

Figure 3: The definition of the SpeciesType class.

\subsubsection{The id and name attributes}

The required id attribute, of type SId, serves to provide a way to identify a speciesType. SpeciesType also has an optional name attribute of type string.

\subsubsection{The compartment attribute}

SpeciesType has an optional attribute compartment, of type SIdRef, to be used to identify the compartment where the speciesType is located. The attribute value must be the identifier of an existing compartment in the model. If present, it must be consistent with the compartment attributes of the referencing species (see Section 3.14 on page 22) and the compartmentReference attributes of its instances (see Section 3.11.3 on page 16). The example in Section 4.1 on page 36 illustrates how to keep the consistency of this attribute.

\subsubsection{ListOfSpeciesFeatureTypes}

ListOfSpeciesFeatureTypes is defined in Figure 3, and is extended from the ListOf class. If present, a ListOfSpeciesFeatureTypes object must have one or more SpeciesFeatureType children. Since ListOfSpeciesFeatureTypes is derived from SBase through ListOf, it inherits the sboTerm and metaid attributes, as well as the optional child Notes and Annotation objects.

\subsubsection{ListOfSpeciesTypelnstances}

ListOfSpeciesTypelnstances is defined in Figure 3, and is extended from the ListOf class. If present, a ListOfSpeciesTypelnstances object must have one or more SpeciesTypelnstance children. Since ListOfSpeciesTypelnstances is derived from SBase through ListOf, it inherits the sboTerm and metaid attributes, as well as the optional child 
Notes and Annotation objects.

\subsubsection{ListOfInSpeciesTypeBonds}

ListOfInSpeciesTypeBonds class is defined in Figure 3 on the previous page, and is extended from the ListOf class. If present, a ListOfInSpeciesTypeBonds object must have one or more InSpeciesTypeBond children. Since ListOfInSpeciesTypeBonds is derived from SBase through ListOf, it inherits the sboTerm and metaid attributes, as well as the optional child Notes and Annotation objects.

\subsubsection{ListOfSpeciesTypeComponentIndexes}

ListOfSpeciesTypeComponentIndexes is defined in Figure 3 on the preceding page, and is extended from the ListOf class. If present, a ListOfSpeciesTypeComponentIndexes object must have one or more SpeciesTypeComponentIndex children. Since ListOfSpeciesTypeComponentIndexes is derived from SBase through ListOf, it inherits the sboTerm and metaid attributes, as well as the optional child Notes and Annotation objects.

\subsubsection{BindingSiteSpeciesType}

BindingSiteSpeciesType inherits the SpeciesType class and is defined in Figure 3 on the previous page. A BindingSiteSpeciesType object is a binding site, and its instance can further define the bindingStatus attribute and can participate a binding internally and explicitly in an InSpeciesTypeBond object, or externally and implicitly defined by an OutwardBindingSite object. A binding site must be an atomic component which means that a BindingSiteSpeciesType object cannot contain a ListOfSpeciesTypelnstances element.

吗安 Note:

In the Multi package, a binding site can only participate in one binding at a time. That means a binding site cannot bind two partners at the same time. Binding is one-to-one at all times.

\subsection{SpeciesFeatureType}

SpeciesFeatureType is defined in Figure 4 on the following page, and serves to provide frameworks or templates to define the referencing SpeciesFeature objects. SpeciesFeatureType has two required attributes id and occur, an optional attribute name, and a required child ListOfPossibleSpeciesFeatureValues element. The multiple PossibleSpeciesFeatureValue elements in the ListOfPossibleSpeciesFeatureValues object permit constructing multistate species via its speciesFeatures under the ListOfSpeciesFeatures or SubListOfSpeciesFeatures object. Since SpeciesFeatureType is derived from SBase, it inherits the sboTerm and metaid attributes, as well as the optional child Notes and Annotation objects.

\subsubsection{The id and name attributes}

The required id attribute, of type SId, serves to provide a way to identify a speciesFeatureType. Its value must be unique within its direct parent speciesType. When a speciesFeatureType is referenced by a speciesFeature, a SpeciesTypeComponentIndex object indexing the containing component can be used to avoid ambiguity.

SpeciesFeatureType also has an optional name attribute of type string.

\subsubsection{The occur attribute}

SpeciesFeatureType has a required attribute occur, of type positiveInteger, used to indicate the number of instances of the speciesFeatureType. This attribute can be used to infer the number of the instances in don't care state with the use of the occur attribute in a referencing speciesFeature (also see Section 3.18.3 on page 26).

\subsubsection{ListOfPossibleSpeciesFeatureValues}

ListOfPossibleSpeciesFeatureValues is defined in Figure 4 on the following page, and is extended from the ListOf class. A list0fPossible- SpeciesFeatureValues must have one or more PossibleSpeciesFeatureValue chil- 


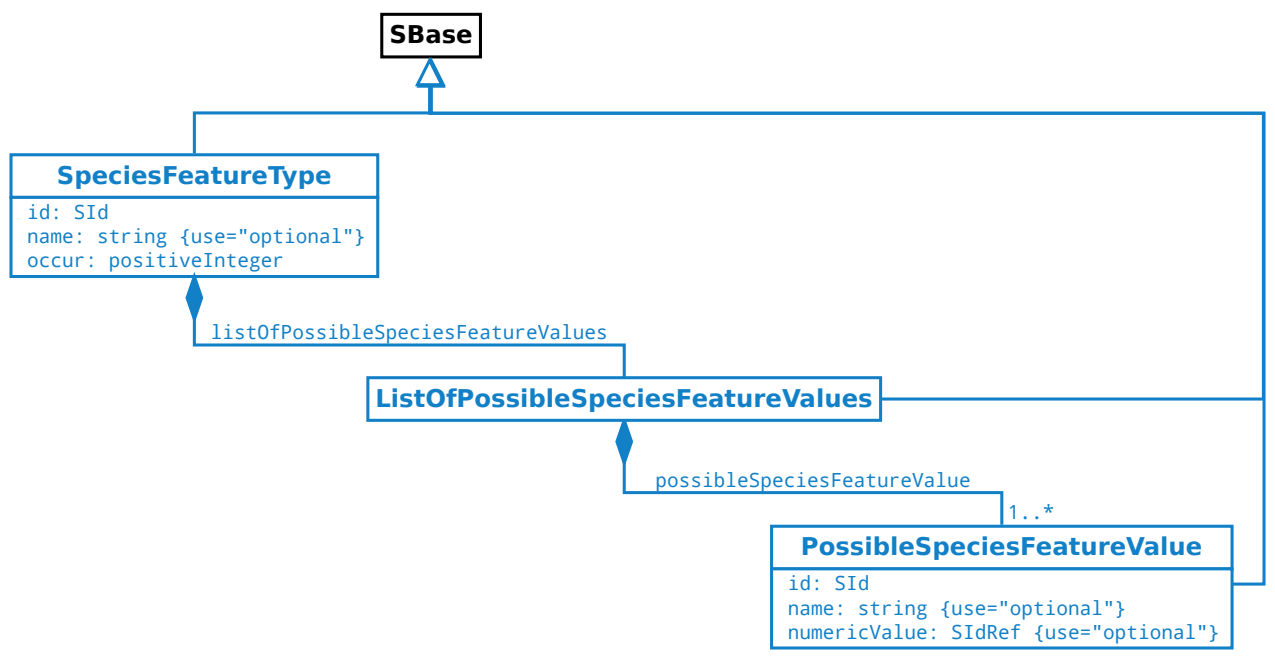

Figure 4: The definitions of SpeciesFeatureType, ListOfPossibleSpeciesFeatureValues and PossibleSpeciesFeature Value classes.

dren. Since ListOfPossibleSpeciesFeatureValues is derived from SBase through ListOf, it inherits the sboTerm and metaid attributes, as well as the optional child Notes and Annotation objects.

\subsection{PossibleSpeciesFeatureValue}

PossibleSpeciesFeatureValue is defined in Figure 4, and is used to define the possible values a speciesFeature can take. It has a required attribute id and two optional attributes name and numericValue. Since PossibleSpeciesFeatureValue is derived from SBase, it inherits the sboTerm and metaid attributes, as well as the optional child Notes and Annotation objects.

\subsubsection{The id and name attributes}

The required id attribute, of type SId, serves to provide a way to identify a possibleSpeciesFeatureValue.

If the id of a possibleSpeciesFeatureValue is the content of a ci element in a MathML expression, it can either represent the numericValue ( when the ci has representationType="numericValue") or the count of the feature instances (default) which have this value.

PossibleSpeciesFeatureValue also has an optional name attribute of type string.

\subsubsection{The numericValue attribute}

PossibleSpeciesFeatureValue has an optional attribute numericValue to be used to provide a reference to a numeric value that the PossibleSpeciesFeatureValue object can have. This attribute has type of SIdRef, and the value must be the identifier of a Parameter object in the model. The numeric value along with the unit can be defined in the Parameter object.

The modeler can either use the identifier of the parameter, or the identifier of the possibleSpeciesFeatureValue (with ci's representationType and speciesReference attribute) as the content of a ci element to represent its value in MathML expressions in SBML. 


\subsection{SpeciesTypelnstance}

SpeciesTypelnstance serves to provide a way to construct speciesTypes and species with multiple components. A speciesType can contain a list of instances of other speciesTypes which can also have their own speciesTypeInstances, so a speciesType has a tree structure. A speciesType cannot contain an instance of any other speciesType that already contains an instance of it. In other words, circular references are not allowed when constructing speciesTypes. For example, if a speciesType "A" contains an instance of another speciesType "B", "B" must not contain an instance of "A" anywhere in the complete structure of "B".

SpeciesTypelnstance is defined in Figure 5. It has two required attributes, id, and speciesType, and two optional attributes name and compartmentReference. Since SpeciesTypelnstance is derived from SBase, it inherits the sboTerm and metaid attributes, as well as the optional child Notes and Annotation objects.

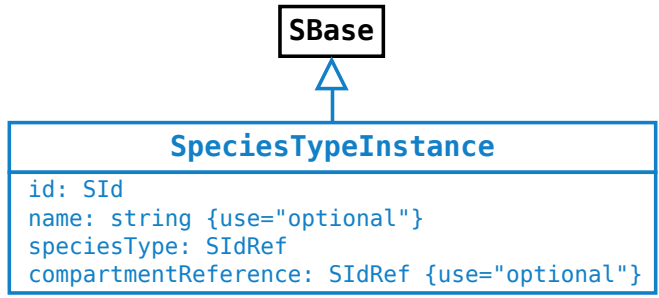

Figure 5: The definition of the SpeciesTypelnstance class

\subsubsection{The id and name attributes}

The required attribute id, of type SId, serves to provide a way to identify a speciesTypeInstance. Its value must be unique within its direct parent speciesType.

SpeciesTypelnstance also has an optional name attribute of type string.

\subsubsection{The speciesType attribute}

The required attribute speciesType, of type SIdRef, is used to reference a speciesType.

\subsubsection{The compartmentReference attribute}

The optional attribute compartmentReference, of type SIdRef, can be used to indicate which sub-compartment in a composite compartment the speciesTypeInstance is located in.

For example, a compartment "cA" has two sub-compartments "cB1" (referenced by compartmentReference "crB1") and "cB2" (referenced by compartmentReference "crB2") of the same compartment type "cB". A speciesType "stA" has two speciesTypeInstances "stiB1" and "stiB2" of the same speciesType "stB". The speciesType "stA" references the compartment "cA" and the speciesType "stB" references the compartment "cB". The speciesTypeInstance "stiB1" is located in "cB1" via the compartmentReference "crB1" and the speciesTypeInstance "stiB2" is located in "cB2" via the compartmentReference "crB2". The SBML code can be as follows:

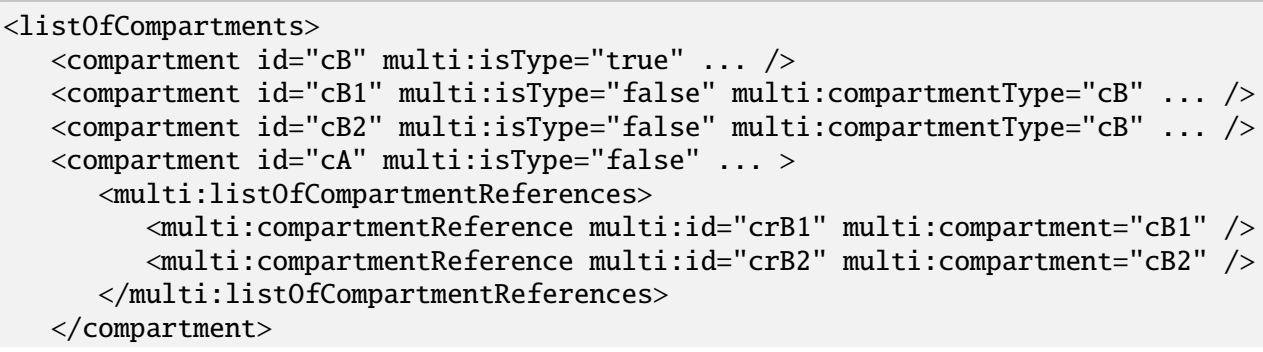




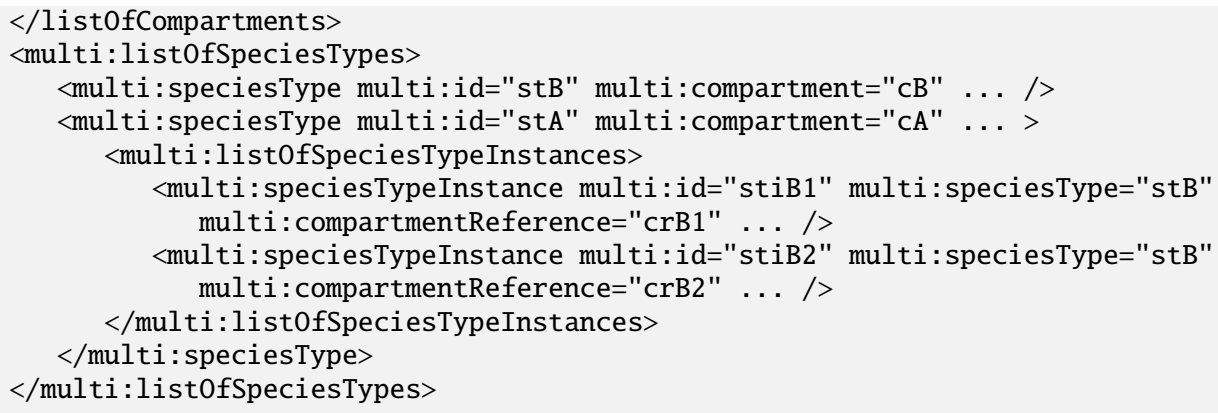

\subsection{SpeciesTypeComponentIndex}

SpeciesTypeComponentIndex provides a way to identify or index a component within a speciesType. A SpeciesTypeComponentIndex object can be referenced by other classes, such as InSpeciesTypeBond, OutwardBindingSite, SpeciesFeature or SpeciesTypeComponentMapInProduct, which needs to identify a component in a particular speciesType.

SpeciesTypeComponentIndex is defined in Figure 6. It has two required attributes, id, and component, and two optional attributes name and identi fyingParent. Since SpeciesTypeComponentIndex is derived from SBase, it inherits the sboTerm and metaid attributes, as well as the optional child Notes and Annotation objects.

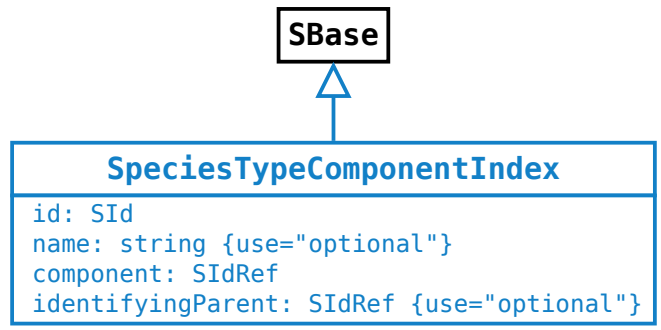

Figure 6: The definition of the SpeciesTypeComponentIndex class

See Section 3.16.3 on page 23 about how to use SpeciesTypeComponentIndex in an outwardBindingSite.

Note:

A speciesTypeComponentIndex should be unambiguous. For example, a speciesTypeComponent Index should not reference to a speciesType which is referenced by two speciesTypeInstances contained in the same SpeciesType object.

\subsubsection{The id and name attributes}

The id attribute, of type SId, provides a way to identify a speciesTypeComponentIndex. The value must be unique within the direct parent speciesType. SpeciesTypeComponentIndex also has an optional name attribute of type string.

\subsubsection{The component attribute}

The component attribute, of type of SIdRef, references a speciesTypeInstance in the speciesType, or the speciesType itself. The value of this attribute can be the id of a speciesTypeInstance or a speciesTypeComponentIndex that is defined in the speciesType of a speciesTypeInstance. 


\subsubsection{The identifyingParent attribute}

The component attribute itself may not be sufficient to uniquely reference a component in a speciesType. The identifyingParent attribute provides assistance for the identification of a component. It references a parent of the component and the value can be the id of a SpeciesTypelnstance object, SpeciesTypeComponentIndex or SpeciesType.

This example illustrates the use of the identifyingParent attribute. There are three speciesTypes "stA", "stB" and "stC". The speciesType "stB" contains two speciesTypeInstances "C1" and "C2" of the same speciesType "stC". The speciesType "stA" contains two speciesTypeInstances "B1" and "B2" of the same speciesType "stB". The speciesType "A" may be required to index every " $\mathrm{C} 1$ " and "C2" by its ListOfInSpeciesTypeBonds child or referencing species. The following SBML code demonstrates how to do the indexing with assistance from the identifyingParent attribute.

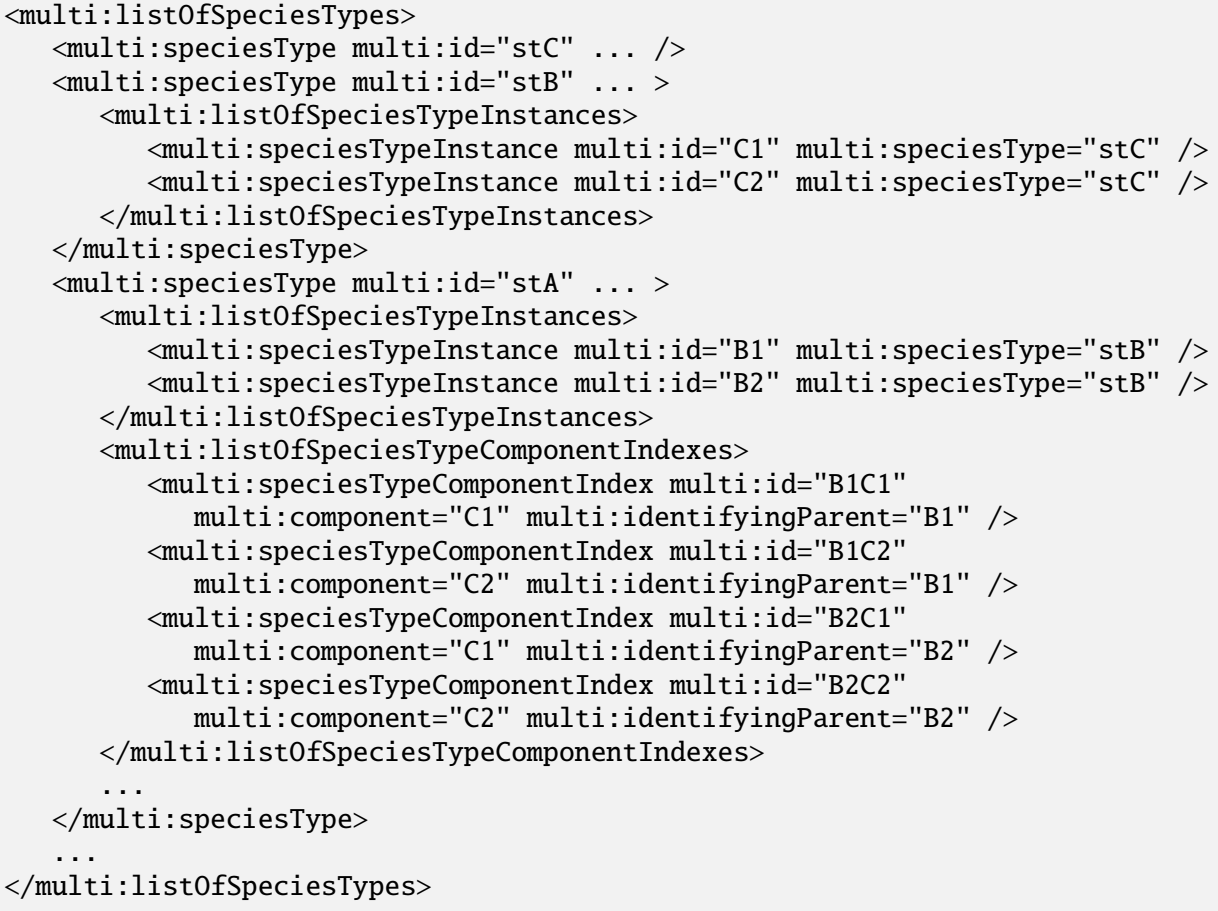

In the speciesType "stA", "B1C1" identifies the "C1" in "B1" and "B2C1" identifies the "C1" in "B2". Similarly, "B1C2" identifies the "C2" in "B1" and "B2C2" identifies "C2" in "B2".

\subsubsection{Reference a component in a speciesType or a species}

In the Multi package, component(s) of a speciesType (or a species via its speciesType attribute) can be referenced by objects of multiple classes such as OutwardBindingSite and SpeciesFeature. A component of a speciesType can be a speciesTypeInstance or the speciesType itself. For example:

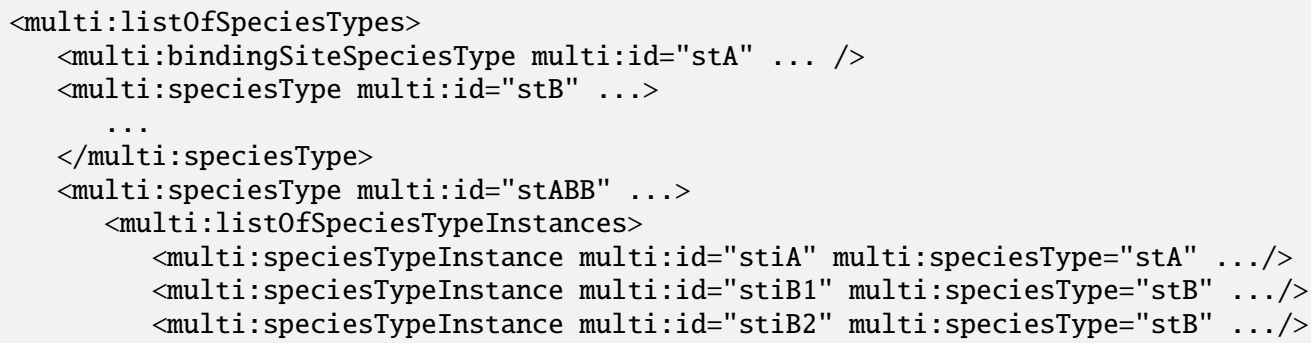




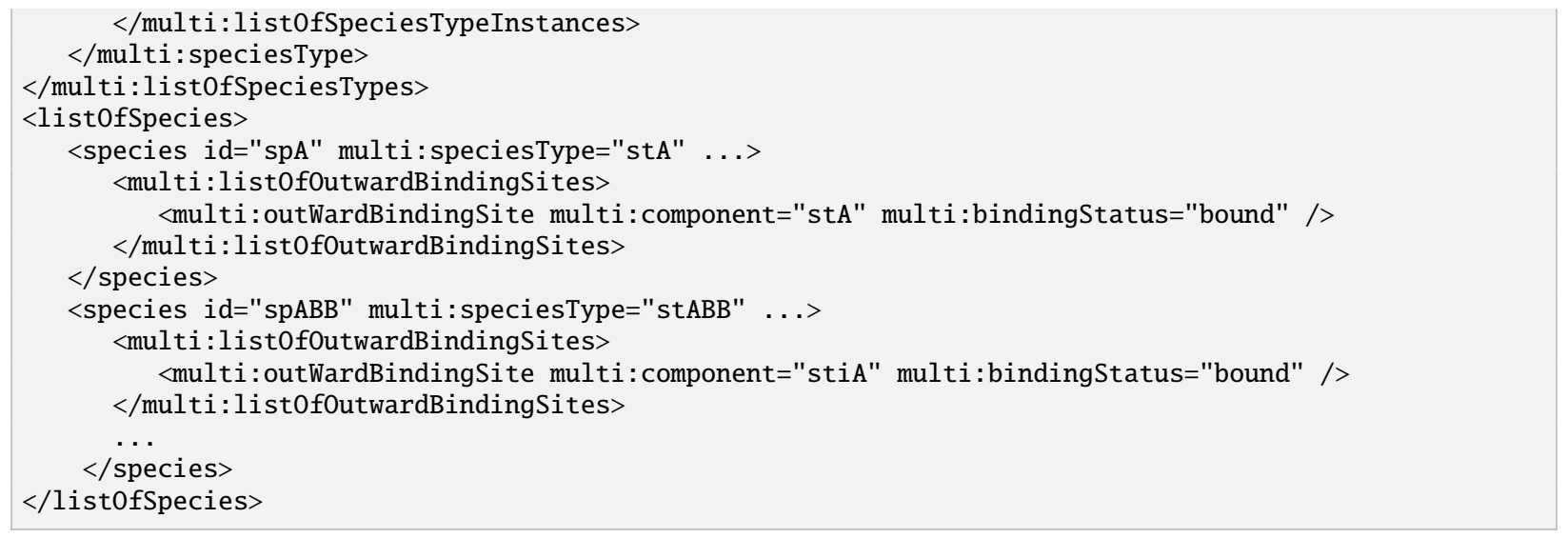

In this example, the component of the outwardBindingSite in species "spABB" is a speciesTypeInstance ("spABB"), and the component of the outwardBindingSite in species"spA" is a speciesType ("stA") which is directly referenced by the speciesType attribute of "spA".

In many cases, to reference a component, the $i d$ of the referenced component will be sufficient and it is not necessary to create an index (speciesTypeComponentIndex). The example in Section 3.12.3 on the previous page illustrates two equivalent ways to reference a component, for example, the "B1" component in the "stA" speciesType. The creation of a speciesTypeComponentIndex cannot be avoided when a speciesType (indirectly) has two speciesTypeInstances that have the same id.

\subsection{InSpeciesTypeBond}

An InSpeciesTypeBond object defines a bond existing within a speciesType. The bond therefore exists in every species that references the speciesType.

InSpeciesTypeBond is defined in Figure 7. It has two optional attributes, id and name, and two required attributes, bindingSite1 and bindingSite2. Since InSpeciesTypeBond is derived from SBase, it inherits the sboTerm and metaid attributes, as well as the optional child Notes and Annotation objects.

The binding relationship in an inSpeciesTypeBond is one-to-one (see Section 3.8.7 on page 14). The uniqueness of an inSpeciesTypeBond is ensured by the pair of referenced bindingSites. A speciesType cannot have two inSpeciesTypeBonds containing the same pair of bindingSites.

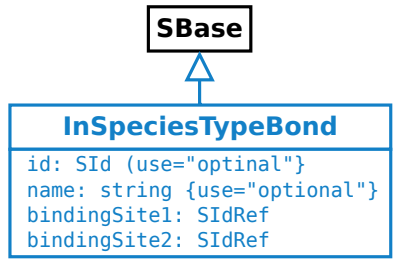

Figure 7: The definition of the InSpeciesTypeBond class

\subsubsection{The id and name attributes}

The optional id attribute, of type SId, provides a way to identify an inSpeciesTypeBond. If present, the value of the id attribute must be unique within its direct parent speciesType.

InSpeciesTypeBond also has an optional name attribute of type string. 


\subsubsection{The bindingSite1 and bindingSite2 attributes}

InSpeciesTypeBond has two required attributes, bindingSite1 and bindingSite2, both of type SIdRef, used to reference a pair of binding sites of the InSpeciesTypeBond object in a speciesType. The referenced identifiers of the binding sites can be the ids of the speciesTypeInstances (binding sites), or the ids of the speciesTypeComponentIndexes indexing the binding sites and the ultimately referenced components must be the BindingSiteSpeciesType objects. Obviously, bindingSite1 and bindingSi te2 must not reference the same BindingSiteSpeciesType object.

\subsection{Uniqueness of SpeciesType definitions}

In some special cases, it may be possible to define a speciesType in multiple equivalent ways.

Figure 8 shows an example of a speciesType constructed in two different ways. The two "st_x" speciesTypes in the diagram can be the results of different reaction paths, but they are equivalent and define the same speciesType.

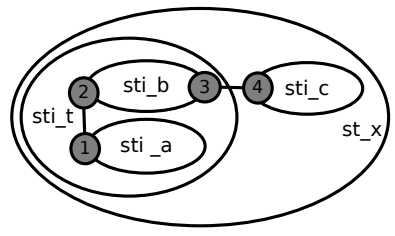

A

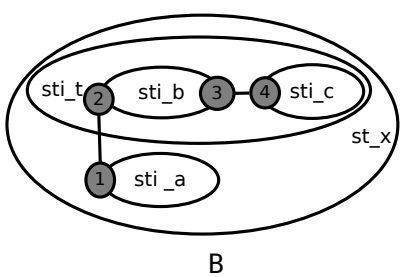

Figure 8: Different formats of the same speciesType

Construct 1: The definition of speciesType "st_x" on the left (A) in Figure 8.

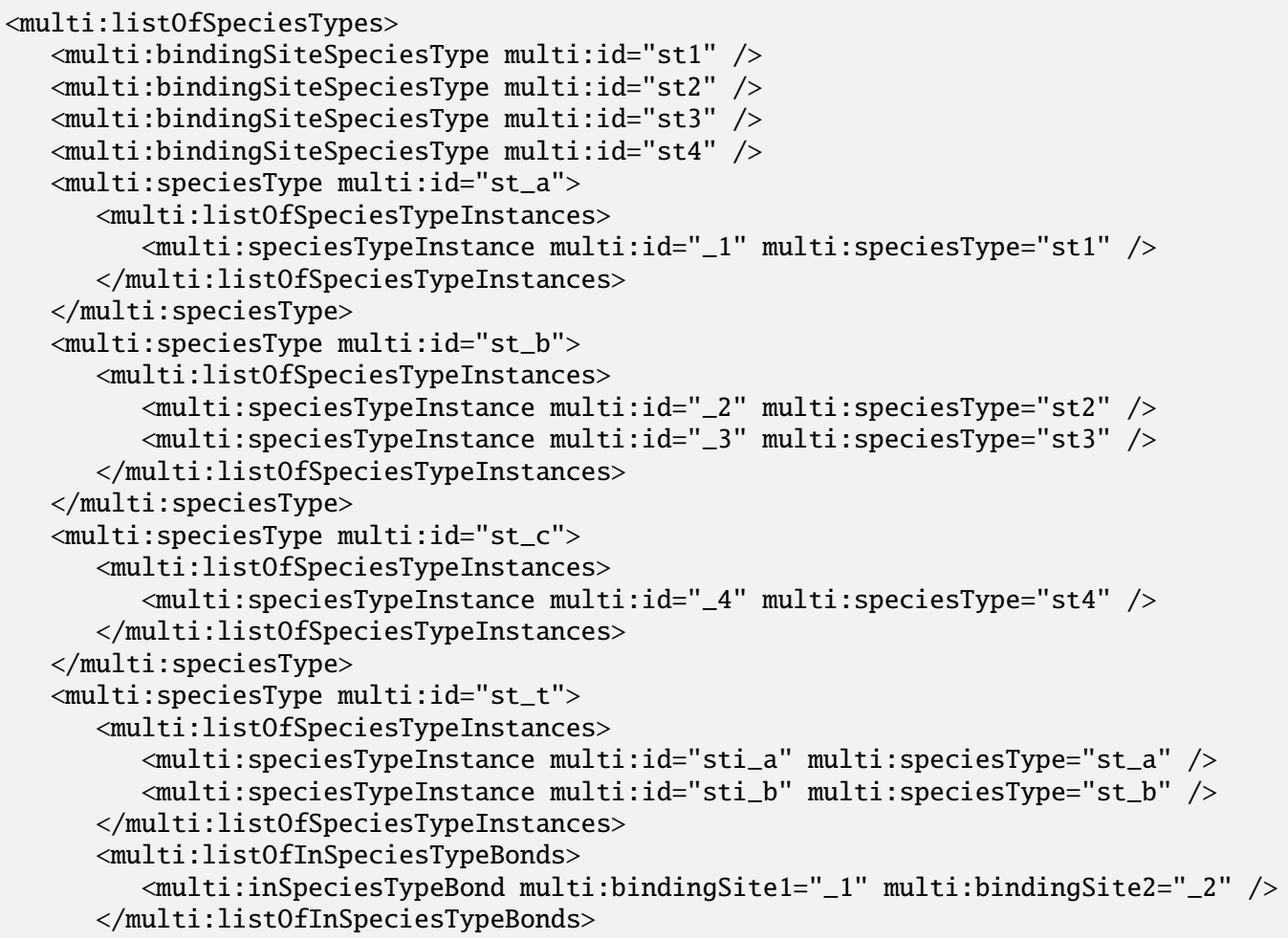


$<$ /multi:speciesType $>$

$<$ multi:speciesType multi:id="st_x">

$<$ multi:list0fSpeciesTypeInstances>

<multi:speciesTypeInstance multi:id="sti_t" multi:speciesType="st_t" />

$<$ multi:speciesTypeInstance multi:id="sti_c" multi:speciesType="st_c" />

$<$ /multi:list0fSpeciesTypeInstances $>$

$<$ multi:list0fInSpeciesTypeBonds>

<multi:inSpeciesTypeBond multi:bindingSite1="_3" multi:bindingSite2="_4" />

$</$ multi:list0fInSpeciesTypeBonds $>$

$</$ multi:speciesType $>$

$</$ multi:listOfSpeciesTypes $>$

Construct 2: The definition of speciesType "st_x" on the right (B) in Figure 8 on the preceding page.

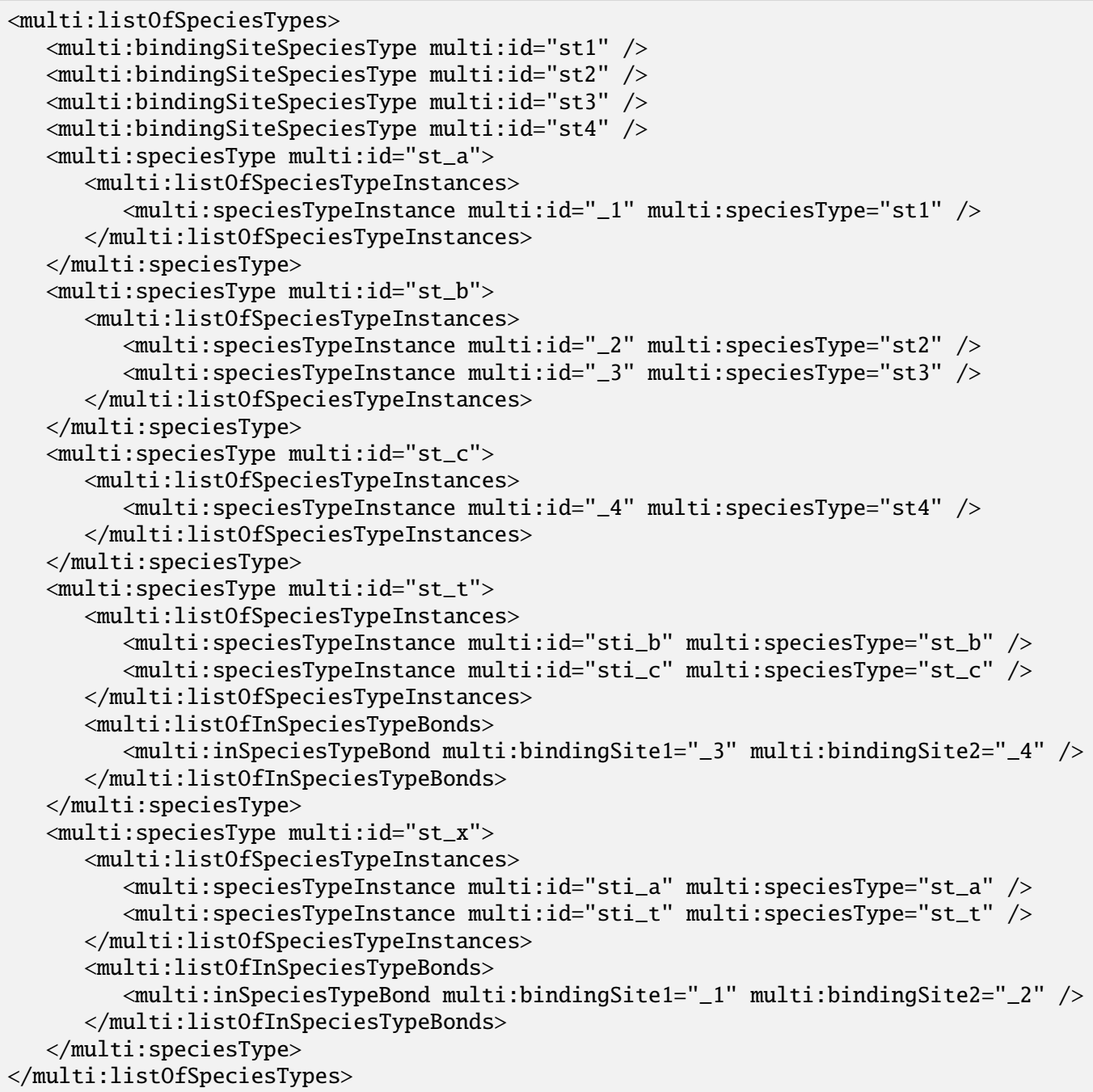

This kind of ambiguity cannot be avoided for speciesTypes involving more than two subcomponents connected by inSpeciesTypeBonds. A and B in Figure 8 on the previous page can be products of different association reactions. It is up to the modeler (parser) to identify whether the two speciesTypes such as those in the example above are identical. 


\subsection{Extended Species}

A species in SBML Level 3 Core refers to a pool of entities. A species in the Multi package is extended from a pool to a template or pattern to which multiple pools may map to. An extended species can reference a speciesType that provides the backbone for the species such as components (including binding sites) and speciesFeatureTypes. When referencing a speciesType, a species can be further defined with regard to the binding statuses of its outwardBindingSites and the speciesFeatures. With the options to have variable values selected, such as "either" for the bindingStatus attribute and multiple possibleSpeciesFeatureValues for a speciesFeature, an extended species can work as a template or pattern how species participate in reactions.

The extension of the Species class is illustrated in Figure 9. It has a new optional attribute speciesType, and two extra optional ListOfOutwardBindingSites and ListOfSpeciesFeatures children. A species may have a ListOfOutwardBindingSites element and/or a ListOfSpeciesFeatures element only when its speciesType attribute has been defined. A species must have its speciesType attribute defined when it has a ListOfOutwardBindingSites element and/or a ListOfSpeciesFeatures element. The relationship among the elements of a ListOfOutwardBindingSites or ListOfSpeciesFeatures object is "and".

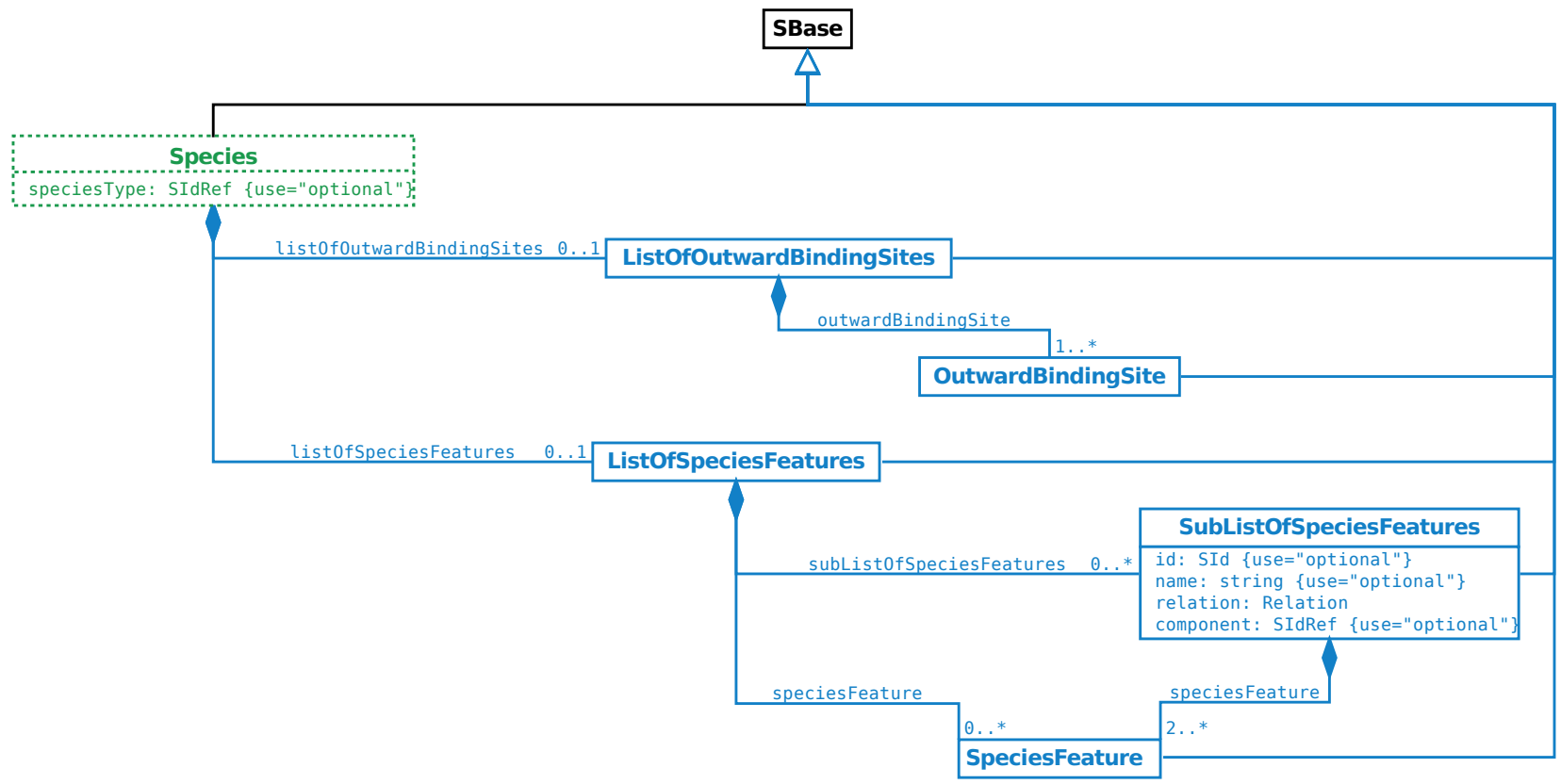

Figure 9: The extension of the Species class

\subsubsection{The speciesType attribute}

The optional attribute speciesType, of type SIdRef, references a SpeciesType object.

\subsubsection{ListOfOutwardBindingSites}

ListOfOutwardBindingSites is defined in Figure 9 and is extended from the ListOf class. A list0fOutwardBindingSites can only be defined when the speciesType attribute is defined. If present, it must have one or more OutwardBindingSite children. Since ListOfOutwardBindingSites is derived from SBase through ListOf, it inherits the sboTerm and metaid attributes, as well as the optional child Notes and Annotation objects. 
sites not involved in any inSpeciesTypeBond) defined by the referenced speciesType. If an outwardBindingSi te is not listed in the listOfOutwardBindingSites, the value of its bindingStatus is "either". In other words, the binding site is in a "don't care" state.

\subsubsection{ListOfSpeciesFeatures}

ListOfSpeciesFeatures is defined in Figure 9 on the preceding page and is extended from the ListOf class. A listOfSpeciesFeatures can only be defined when the speciesType attribute is defined. If present, it must have one or more children. A child can be a SpeciesFeature, or a SubListOfSpeciesFeatures object.

note:

The list0fSpeciesFeatures of a species does not have to cover all the speciesFeatures corresponding to all speciesFeatureTypes (see Section 3.9 on page 14) of every component defined by the referenced speciesType. If $a$ speciesFeatureType is defined and there is no speciesFeature explicitly referencing $i t$, the species has an implicit speciesFeature having all the list0fPossibleSpeciesFeatureValues and "or" relationships between them. In other words, the implicit speciesFeature has a "don't care" state.

Since ListOfSpeciesFeatures is derived from SBase through ListOf, it inherits the sboTerm and metaid attributes, as well as the optional child Notes and Annotation objects.

The example at Section 3.18.7 on page 27 illustrates the usage of the ListOfSpeciesFeatures class.

\subsection{OutwardBindingSite}

OutwardBindingSite is defined in Figure 10. It has two optional attributes, id and name, and two required attributes, bindingStatus and component. A binding site not involved in any InSpeciesTypeBond object in the speciesType referenced by a species is an outwardBindingSite. Since OutwardBindingSite is derived from SBase, it inherits the sboTerm and metaid attributes, as well as the optional child Notes and Annotation objects.

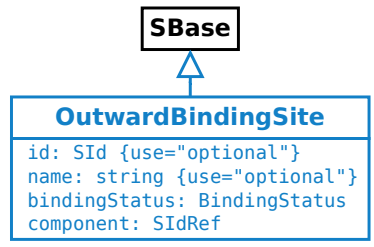

Figure 10: The definition of the OutwardBindingSite class

\subsubsection{The id and name attributes}

The optional id attribute, of type SId, can serve to provide a way to identify an outwardBindingSite. If present, the value must be unique within the species. OutwardBindingSite also has an optional name attribute of type string.

\subsubsection{The bindingStatus attribute}

The bindingStatus attribute takes a value of type BindingStatus.

\subsubsection{The component attribute}

The component attribute, of type SIdRef, references a component which ultimately references a BindingSiteSpeciesType object. The attribute value must be the identifier of a SpeciesTypelnstance, SpeciesTypeComponentIndex or SpeciesType object. An outwardBindingSi te cannot be a binding site referenced by any inSpeciesTypeBond in the species. 
There are three scenarios for the component attribute to have its value as an identifier of a SpeciesType, SpeciesTypelnstance, or SpeciesTypeComponentIndex object.

(1) When a species references a bindingSiteSpeciesType, the component attribute of its outwardBindingSite can only have value of the referenced speciesType's id.

(2) When a species references a speciesType with a speciesTypeInstance being a binding site (have an id of BindingSiteSpeciesType as its speciesType attribute) and the id of the speciesTypeInstance can identify the binding site within the speciesType (referenced by the species) unambiguously, and therefore, an outwardBindingSite of the species can have its component attribute with value of a speciesTypeInstance's id.

(3) When a species references a speciesType with a speciesTypeInstance being a binding site (directly or indirectly) and id of the speciesTypeInstance can NOT identify the binding site without ambiguity, an id of SpeciesTypeComponentIndex can be used as the value of the component attribute for an outwardBindingSite of the species.

\subsubsection{Example}

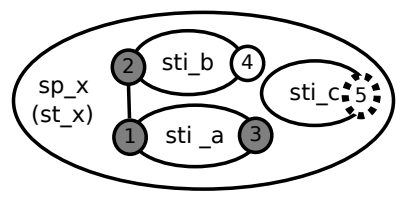

Figure 11: An example of OutwardBindingSite

Figure 11 illustrates the usage of the OutwardBindingSite class. Species "sp_x" references speciesType "st_x", which has three speciesTypeInstances "sti_a", "sti_b" and "sti_c". SpeciesTypeInstance "sti_a" has bindingSites “_1" and “_3”, speciesTypeInstance "sti_b” has bindingSites “_2” and “_4”, and speciesTypeInstance "sti_c" has bindingSite "_5". The inSpeciesTypeBond in "st_x" involves two bindingSites "_1" and “_2". The other three bindingSites, “_3”, “_4” and “_5”, in the species "sp_x” are outwardBindingSites. The outwardBindingSite "_3" is "bound" (filled circle with solid line in the diagram), the outwardBindingSite "_4" is "unbound" (empty circle with solid line) and the outwardBindingSite "_5" has binding status "either" (empty circle with dotted line). The corresponding SBML code would be as follows:

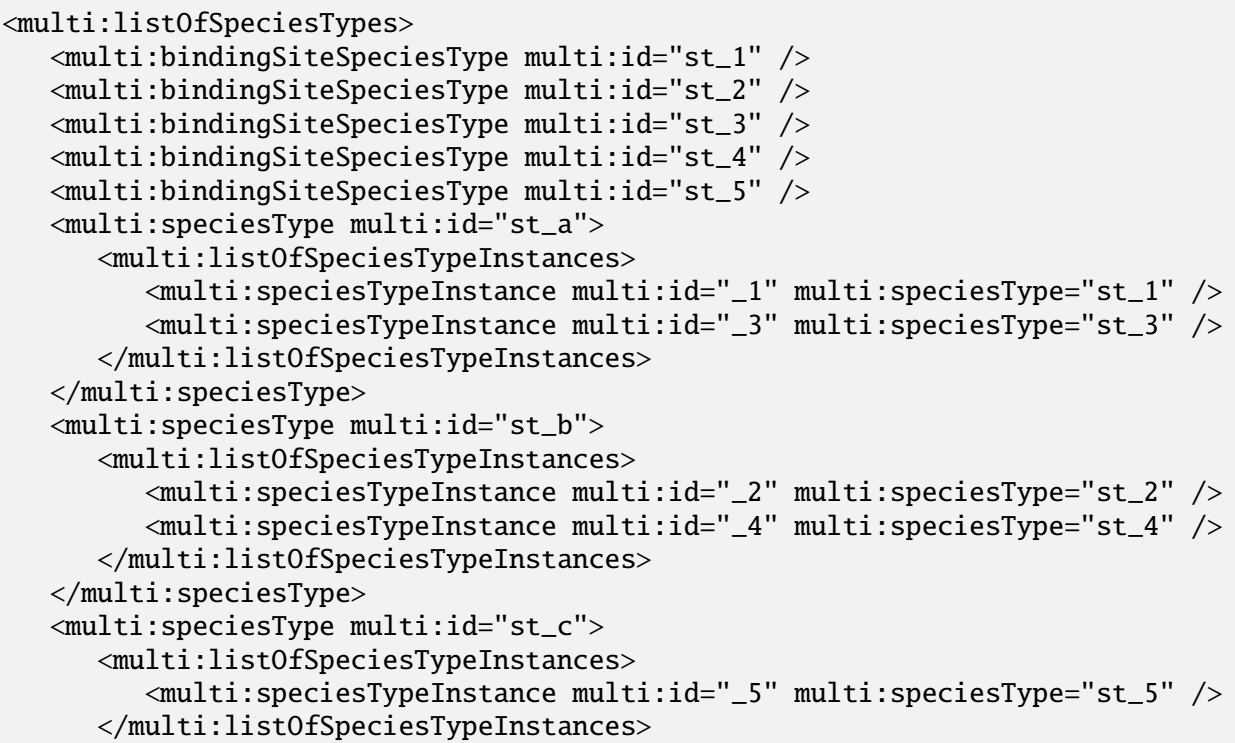




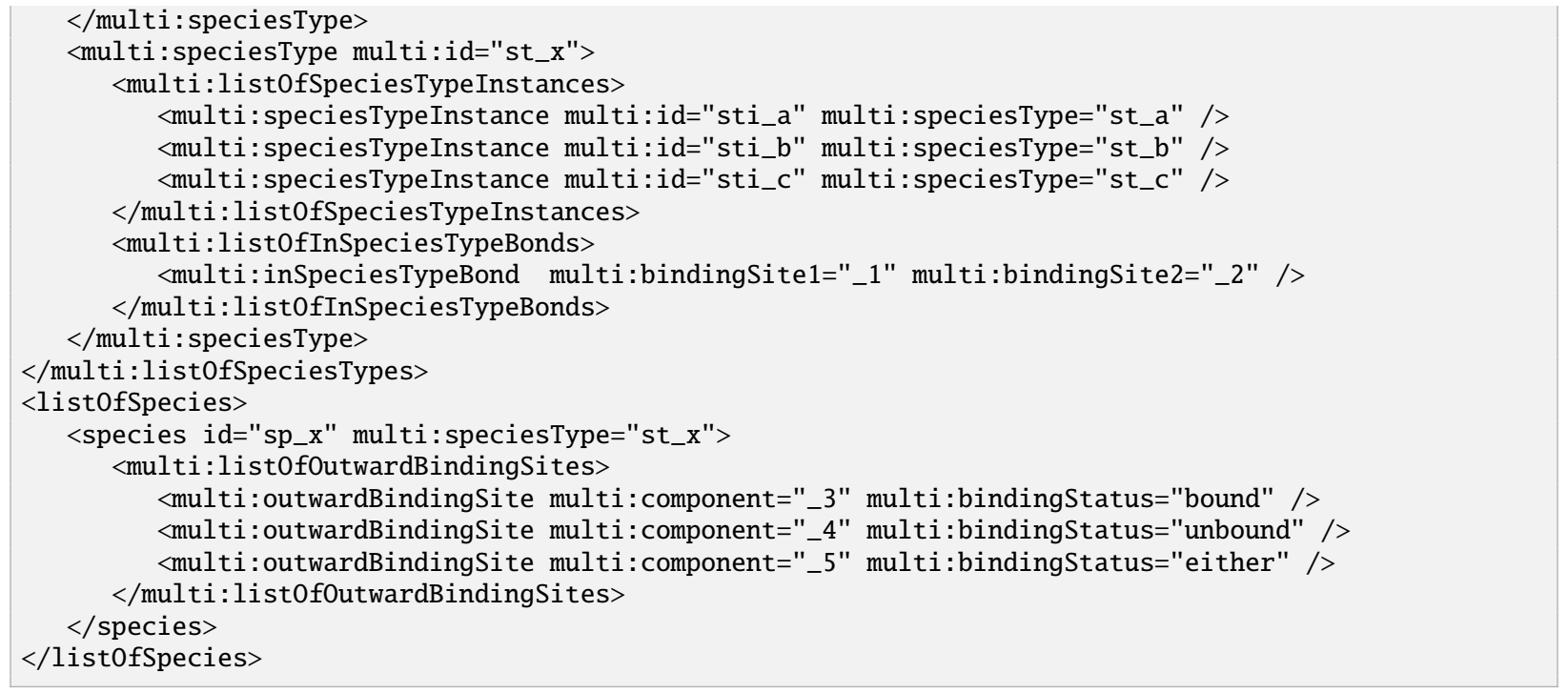

\subsection{SubListOfSpeciesFeatures}

SubListOfSpeciesFeatures is defined in Figure 9 on page 22, and is extended from the ListOf class. A SubListOfSpeciesFeatures object must have two or more SpeciesFeature children. Since SubListOfSpeciesFeatures is derived from SBase through ListOf, it inherits the sboTerm and metaid attributes, as well as the optional child Notes and Annotation objects.

\subsubsection{The id and name attributes}

The optional id attribute, of type SId, can serve to provide a way to identify a SubListOfSpeciesFeatures object. If present, its value must be unique within the species. SubListOfSpeciesFeatures also has an optional name attribute of type string.

\subsubsection{The relation attribute}

SubListOfSpeciesFeatures has a required attribute relation, of type Relation, to define the logic relationship among its children. If any speciesFeature involved in a subList0fSpeciesFeatures references a speciesFeatureType with "occur > 1", the subList0fSpeciesFeatures can only have the value "and" for its relation attribute.

\subsubsection{The component attribute}

The optional component attribute, of type SIdRef, can be used to indicate which component in a species the SubListOfSpeciesFeatures object belongs to. It is required when a speciesFeature contained in this subList0fSpeciesFeatures cannot be identified only based on its speciesFeatureType attribute.

\subsection{SpeciesFeature}

SpeciesFeature is defined in Figure 12 on the following page. It has three optional attributes, id, name and component, and two required attributes, speciesFeatureType and occur, and a required child list0fSpeciesFeatureValues. Since SpeciesFeature is derived from SBase, it inherits the sboTerm and metaid attributes, as well as the optional child Notes and Annotation objects. SpeciesFeature serves to define the state of a component in a species by selecting values from the list0fPossibleSpeciesFeatureValues of the referenced speciesFeatureType. 


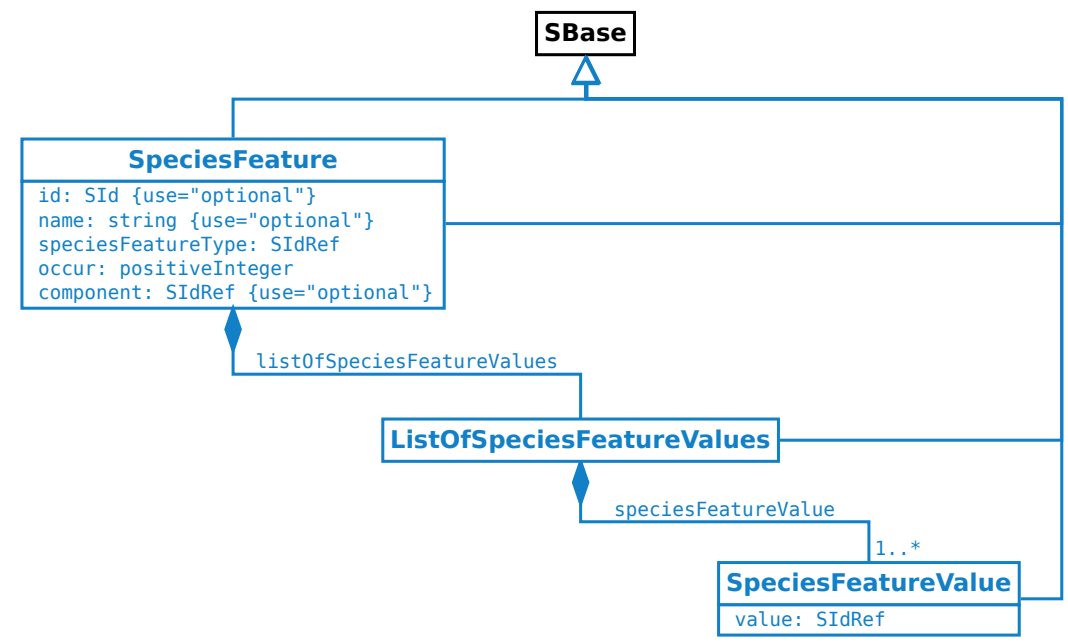

Figure 12: The definitions of the SpeciesFeature class and the SpeciesFeatureValue class

\subsubsection{The id and name attributes}

The optional id attribute, of type SId, can serve to provide a way to identify a speciesFeature. If present, the value must be unique within the species. SpeciesFeature also has an optional name attribute of type string.

\subsubsection{The speciesFeatureType attribute}

SpeciesFeature has a required attribute speciesFeatureType, of type SIdRef, used to reference a speciesFeatureType.

\subsubsection{The occur attribute}

SpeciesFeature has a required attribute occur, of type of positiveInteger, used to define the number of instances of the referenced speciesFeatureType.

The value of the occur attribute cannot be larger than the occur of the referenced speciesFeatureType. When a speciesFeatureType has multiple instances (speciesFeatureType's occur > "1"), the speciesFeature's occur attribute provides a way for a species to define the instances of the speciesFeatureType differently.

For example, in a speciesType, speciesFeatureType "ftA" has occur="2" and two possibleSpeciesFeatureValues "fva1" and "fva2". A species referencing the speciesType can be defined to have two speciesFeatures "sfA1" and "sfA2" both referencing " $f t A$ ". The speciesFeature "sfA1" has occur="1" and its value is "fva1". The speciesFeature "sfA2" has occur="1" and its value is "fva2".

If the occur of a speciesFeature is less than the occur of the referenced speciesFeatureType, the rest of the unspecified instances of the speciesFeatureType are in don't care state which means that the value of an unspecified instance can be any from the list0fPossibleSpeciesFeatureValues.

For example, in a speciesType, a speciesFeatureType "phosphorylation" has two possibleSpeciesFeatureValues "phosphorylated" and "unphosphorylated" and the occur is " 5 ". A species referencing the speciesType can be defined to have a speciesFeature of the "phosphorylation" with the value of "phosphorylated" and the occur of " 1 ". Then, the species is a pattern species with at least one "phosphorylated" site (the other four "phosphorylation" sites are in don't care state). This pattern species can be mapped by anyone of the fully defined species (see Section 3.19 on page 29) of the same type and with any of " 1 " to " 5 " phosphorylated sites. 


\subsubsection{The component attribute}

The optional component attribute, of type SIdRef, can be used to indicate which component in a species the speciesFeature belongs to. It is required when the component cannot be identified only based on the speciesFeatureType attribute.

\subsubsection{ListOfSpeciesFeatureValues}

ListOfSpeciesFeatureValues is defined in Figure 12 on the preceding page, and is extended from the ListOf class. A ListOfSpeciesFeatureValues object must have one or more SpeciesFeatureValue children. If a list0fSpeciesFeatures has multiple speciesFeatureValues, the interpretation of the relationship between them is "or". Since ListOfSpeciesFeatureValues is derived from SBase through ListOf, it inherits the sboTerm and metaid attributes, as well as the optional child Notes and Annotation objects.

\subsubsection{SpeciesFeatureValue}

SpeciesFeatureValue is defined in Figure 12 on the previous page. A speciesFeatureValue serves to specify a value for a speciesFeature to select from the list0fPossibleSpeciesFeatureValues defined in the referenced speciesFeatureType. The SpeciesFeatureValue class has only one attribute value, of type SIdRef, used to reference a PossibleSpeciesFeatureValue object. Since SpeciesFeatureValue is derived from SBase, it inherits the sboTerm and metaid attributes, as well as the optional child Notes and Annotation objects.

\subsubsection{Example}

Figure 13 on the following page is an example speciesType to illustrate the usage of the ListOfSpeciesFeatures, SubListOfSpeciesFeatures and SpeciesFeature classes. SpeciesType "st_X" has speciesTypeInstance "sti_A" with speciesFeatureType "fA", and speciesTypeInstance "sti_B" with speciesFeatureTypes "fB1" and " $f B 2$ ”. The speciesFeatureType "fA" has two possibleSpeciesFeatureValues "v1" and "v2". The speciesFeatureType "fB1" has "v3" and "v4", and "fB2" has "v5" and "v6". Here are several ways to construct the list0fSpeciesFeatures of a species referencing the speciesType "st_X":

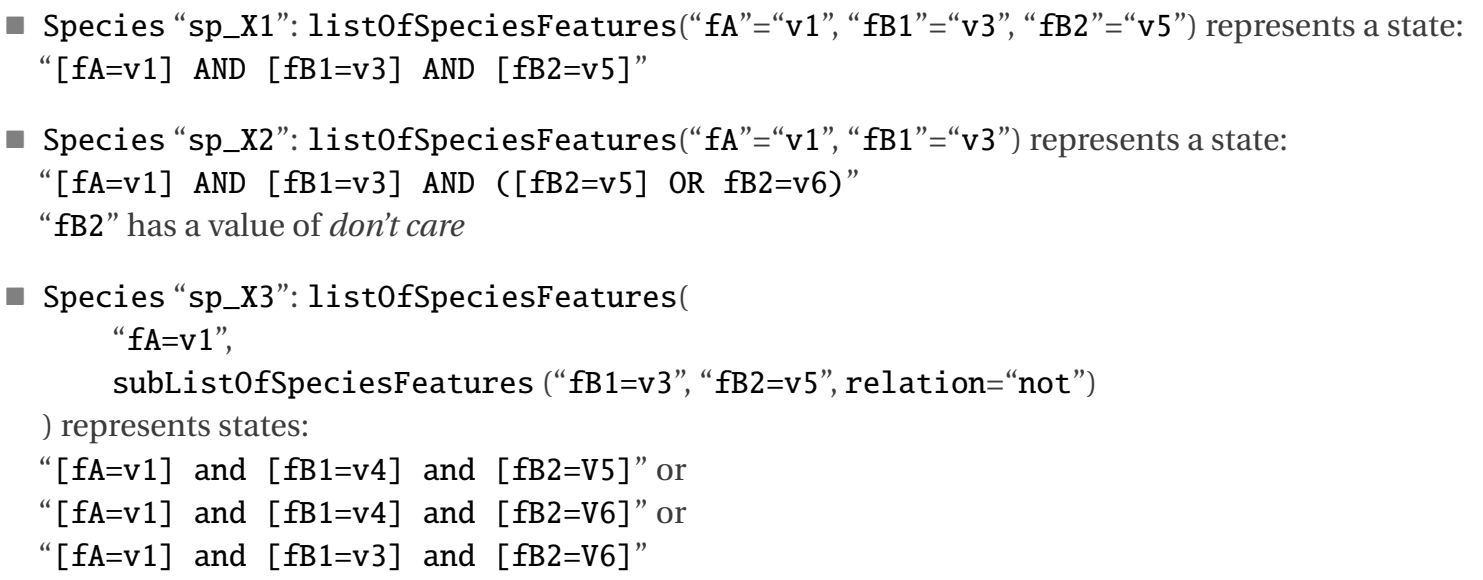




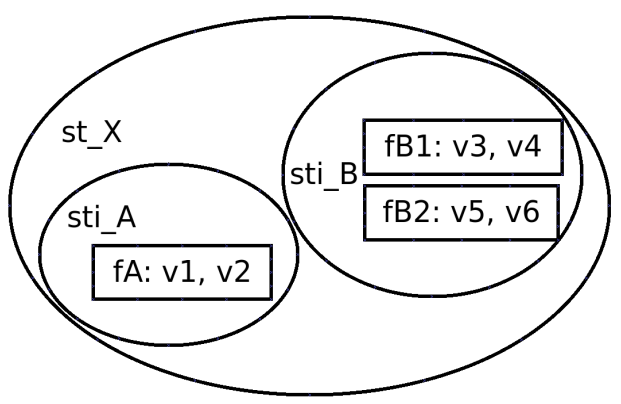

Figure 13: An example speciesFeatureType to illustrate the usage of ListOfSpeciesFeatures, SubListOfSpeciesFeatures and the SpeciesFeature

The SBML code for the speciesTypes "st_A", "st_B", and "st_X", and the species "sp_X3" can be as follows:

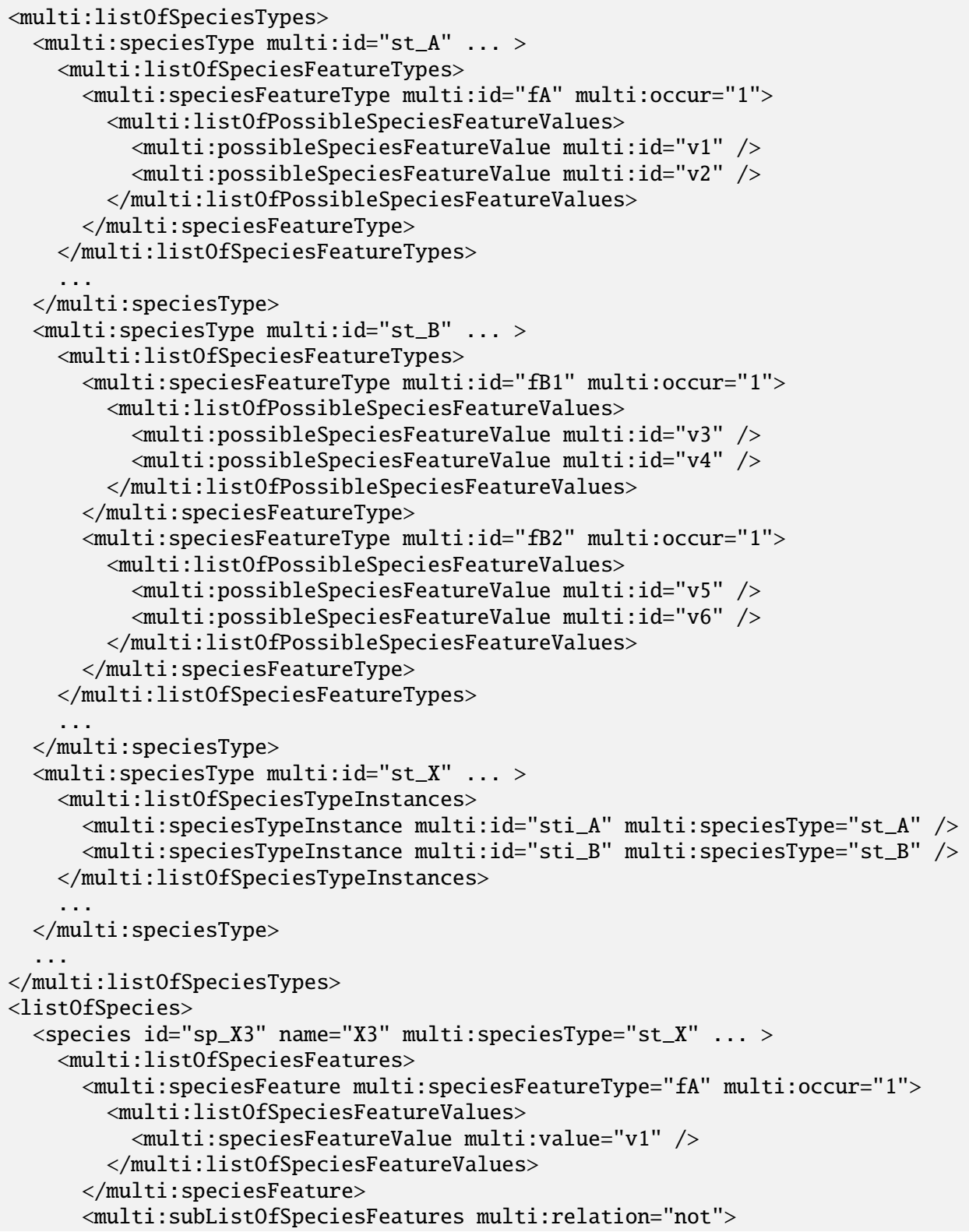




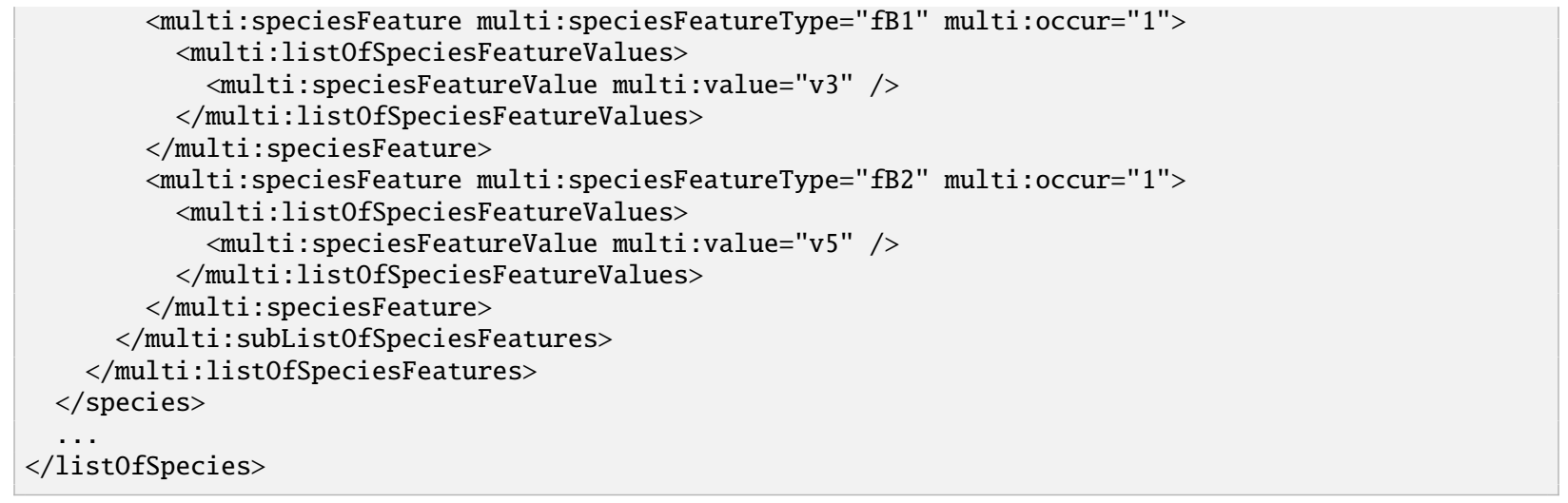

\subsection{Fully defined species and mapping to pattern species}

An extended Species object functions as a template or a pattern which allows multiple pools of entities to map to it. A species is fully defined if there is only one pool mapping to it. A fully defined species can be considered the same as an SBML core species, and can be initialized with the initialAmount attribute, or the initialConcentration attribute, or via an InitialAssignment object. In the Multi package, a species is fully defined if the following conditions are fulfilled:

- All outwardBindingSites must be free (bindingStatus="unbound"), since "bound" sites imply that there is a non-specified binding partner.

- Each speciesFeature occurrence can only have one speciesFeatureValue, and every occurrence of every speciesFeatureType of every component of the referenced speciesType must be referenced by exactly one speciesFeature occurrence.

• Only "and" values are allowed for the relation attributes of the SubListOfSpeciesFeatures objects.

- Only one single SpeciesFeatureValue object is allowed for any speciesFeature.

- The referenced compartment cannot be a compartment type, which means the value of the isType attribute of the referenced compartment can only be "false".

The mapping from a fully defined species to a pattern species is implicit and can be inferred from the structure of the species. For example, a speciesType "stA" has one speciesFeatureType with two possibleSpeciesFeatureValues "v1" and "v2". The species "spA1" references "stA" and has the speciesFeature with the value of "v1". Another species "spA" also references "stA" and has no speciesFeature explicitly defined. Thus, the species "spA1" is a fully defined species and can map to the pattern species "spA" because species "spA" has an implicit speciesFeature which can take either value "v1" or value "v2" (see the note in Section 3.15.3 on page 23).

\subsection{Reaction}

Reaction itself in the Multi package is not extended, but it allows to use the Multi Species objects to construct reactions. The Reaction class in the Multi package cannot only define the relations among pools (SBML core species), but also the relations among patterns (Multi extended species). Several related classes including SimpleSpeciesReference and SpeciesReference are extended to handle some requirements specific to the Multi package. A new class, IntraSpeciesReaction, is derived from Reaction to explicitly define those reactions within the same Species object.

The changes under the Reaction class in the Multi package are illustrated in Figure 14 on the following page. 


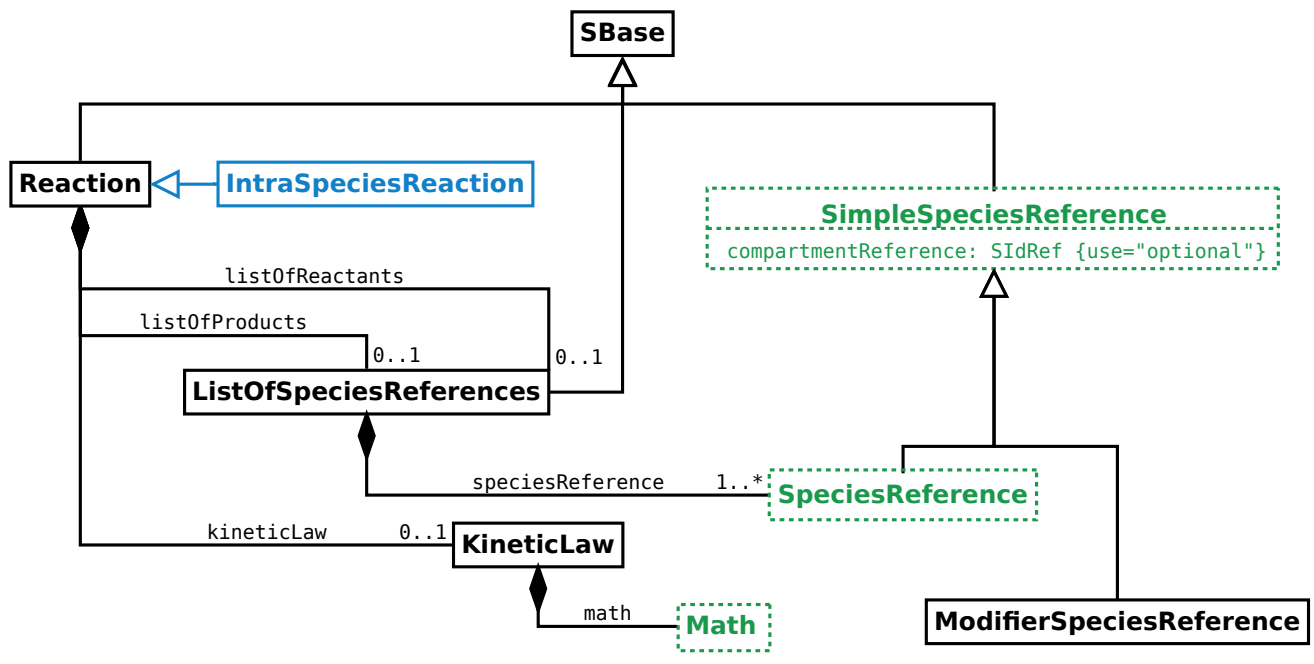

Figure 14: The changes under the Reaction class including IntraSpeciesReaction, SimpleSpeciesReference, SpeciesReference and Math

\subsection{IntraSpeciesReaction}

IntraSpeciesReaction is derived from Reaction for the reactions happening within a species (see the example "Extended Reaction class" at page 23 of the slides at HARMONY 2013 [Zhang and Meier-Schellersheim (2013b)]).

A particular reaction may happen within a species as an intraSpeciesReaction if the following conditions are fulfilled:

- The reaction is either an association reaction or a dissociation reaction.

- If it is an association reaction, each of the two reactant species has at least one outwardBindingSite free ("unbound").

- If it is a dissociation reaction, each of the two product species has at least one outwardBindingSite free ("unbound").

12 Note:

Technically, transformations are reactions only happening in one species. They do not have the ambiguity and, therefore, do not have to be defined as intraSpeciesReactions.

\subsection{Extended SimpleSpeciesReference}

The SimpleSpeciesReference class is extended with a new optional attribute compartmentReference, of type SIdRef, to reference a compartmentReference. The compartmentReference attribute can serve to indicate in which sub-compartment an object of a class (SpeciesReference or ModifierSpeciesReference) inheriting SimpleSpeciesReference is located.

This example illustrates the use of the compartmentReference attribute in simpleSpeciesReferences. The situation described here could correspond to interactions among species located on two adjacent membranes. A model has a composite compartment "cc" with two compartmentReferences "cr1" and "cr2", and both reference "c" subcomponents. Species "spA" references compartment "c", and species "spAA" references the composite compartment "cc". A reaction happens between two "spA" species from the two "c" compartments and results in a cross-compartment product. 


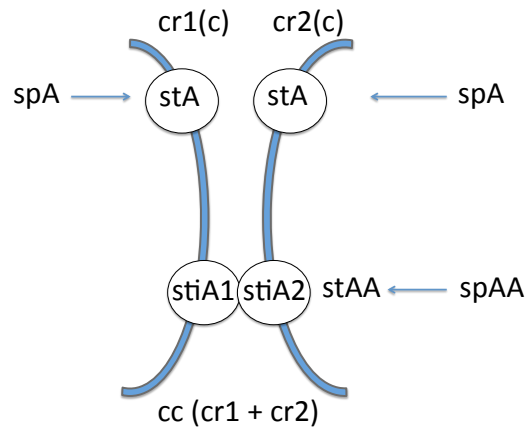

Figure 15: Reaction: $s p A(c r 1)+s p A(c r 2) \rightarrow s p A A$

The SBML code can be as follows:

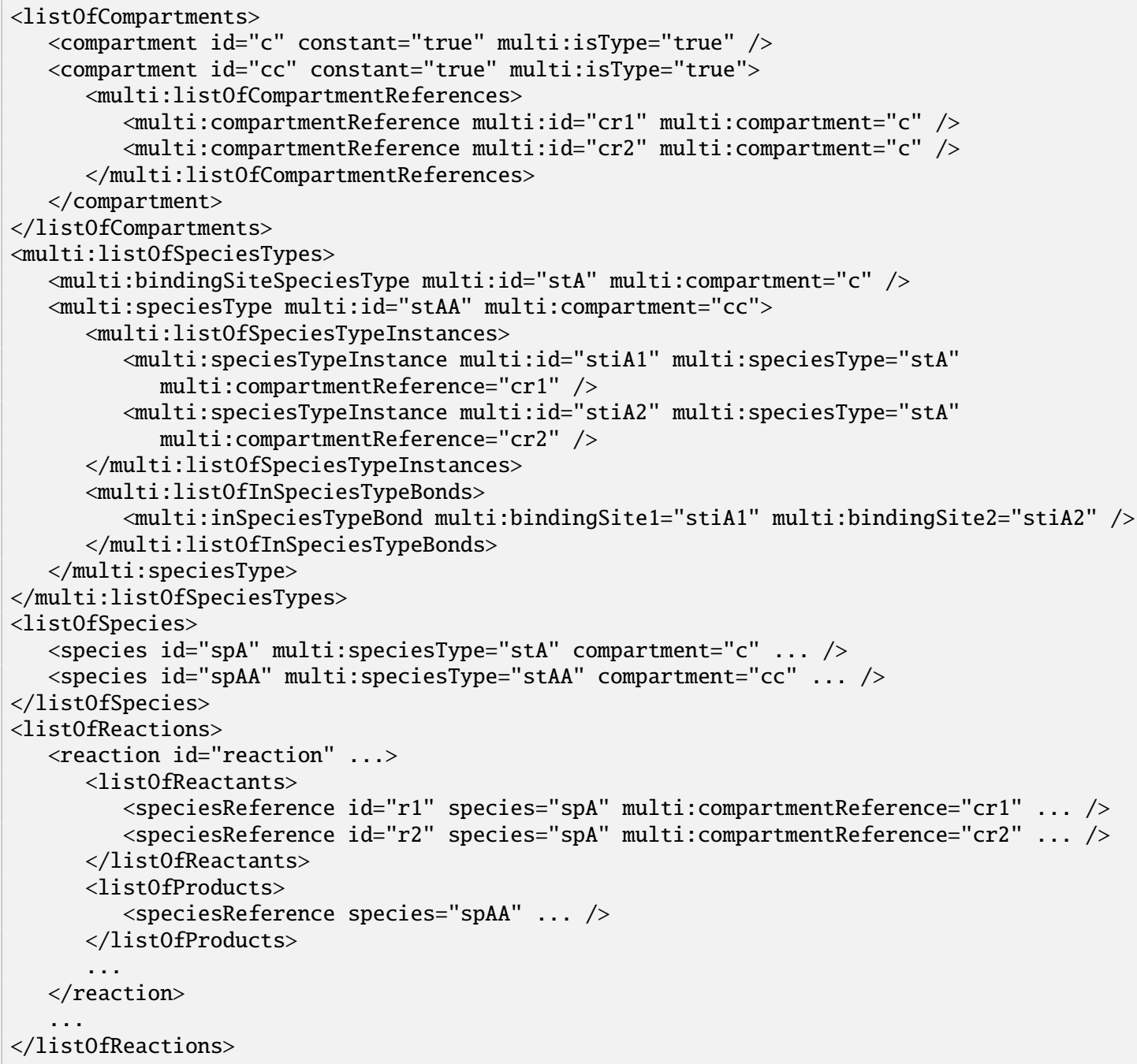




\subsection{Extended SpeciesReference}

The SpeciesReference class is extended from SBML Level 3 Core and can establish component mappings between the reactant species and the product species when the mappings cannot be inferred from the ids of the SpeciesTypelnstance objects. The SpeciesReference class has an optional ListOfSpeciesTypeComponentMapsInProduct child, as defined in Figure 16. Only a reaction product can contain the ListOfSpeciesTypeComponentMapsInProduct child and it is not necessary to store the mappings again in the reactants.

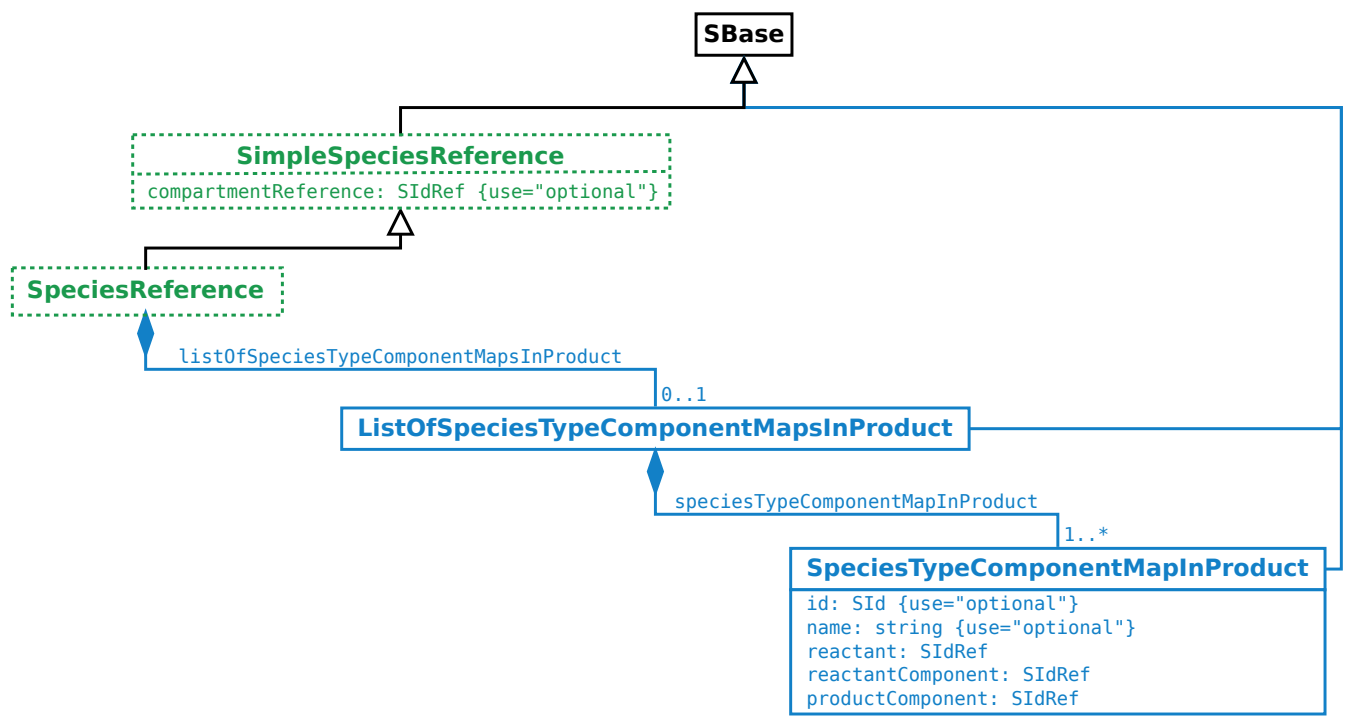

Figure 16: The extension of the SpeciesReference class

\subsubsection{ListOfSpeciesTypeComponentMapsInProduct}

ListOfSpeciesTypeComponentMapsInProduct is defined in Figure 16, and is extended from the ListOf class. A ListOfSpeciesTypeComponentMapsInProduct object must have one or more SpeciesTypeComponentMapInProduct children. Since ListOfSpeciesTypeComponentMapsInProduct is derived from SBase through ListOf, it inherits the sboTerm and metaid attributes, as well as the optional child Notes and Annotation objects.

\subsection{SpeciesTypeComponentMapInProduct}

SpeciesTypeComponentMapInProduct is defined in Figure 16. It has two optional attributes, id and name, and three required attributes, reactant, reactantComponent and productComponent. Since SpeciesTypeComponentMapInProduct is derived from SBase, it inherits the sboTerm and metaid attributes, as well as the optional child Notes and Annotation objects.

A speciesTypeComponentMapInProduct defines the mapping between a component in a reactant and a component in a product. The identifications of a component and the speciesReference should be sufficient to identify the component in the context of a reaction. The attributes reactant and reactantComponent can identify the component in a reactant, and the productComponent attribute and the product storing the mapping information can identify the component in a product.

\subsubsection{The id and name attributes}

The optional id attribute, of type SId, can serve to provide a way to identify a speciesTypeComponentMapInProduct. If present, the value must be unique within the reaction. SpeciesTypeComponentMapInProduct also has an optional name attribute of type string. 


\subsubsection{The reactant attribute}

SpeciesTypeComponentMapInProduct has a required reactant attribute, of type SIdRef, to reference the id of a reactant speciesReference in a reaction.

\subsubsection{The reactantComponent attribute}

SpeciesTypeComponentMapInProduct has a required reactantComponent attribute, of type SIdRef, to reference a component in a reactant species.

\subsubsection{The productComponent attribute}

SpeciesTypeComponentMapInProduct has a required productComponent attribute, of type SIdRef, to reference a component in a product species.

\subsection{OutwardBindingSites and speciesFeatures in don't care state in reac- tion products}

An outwardBindingSite is in don't care state if its bindingStatus is "either" or is not specified (also see Section 3.15.2 on page 22). A speciesFeature is in don't care state if it is not specified in the referencing species (also see Section 3.18 on page 25).

For a species as a product in a reaction, if it has don't care outwardBindingSites or don't care speciesFeatures, the interpretation of the don't care is don't change. In a product, a don't care outwardBindingSite has the same bindingStatus as the mapped outwardBindingSite in the reactant, and a don't care speciesFeature has the same value as the mapped speciesFeature in the reactant.

\subsection{Extended ci elements in Math objects}

The Multi package extends the ci element in Math in Reaction with optional attributes speciesReference and representationType.

\subsubsection{The speciesReference attribute}

The optional speciesReference attribute, of type SIdRef, can only be used when the content of the ci element is a species $i d$, or when the content of the ci element is a speciesFeature $i d$. The speciesReference attribute can identify which species is referenced in a reaction, and the speciesReference attribute must have a value of a speciesReference id within the same reaction.

If the ci content references a species' id, the id represents the concentration or amount of the species.

If the ci content references a speciesFeature's id, the id represents the count of the speciesFeature instances with the speciesFeatureValue (also see Section 3.18.1 on page 26).

The example in Section 3.22 on page 30 can be further extended with a block of kineticLaw in the reaction to illustrate the use of the speciesReference attribute with a species' id.

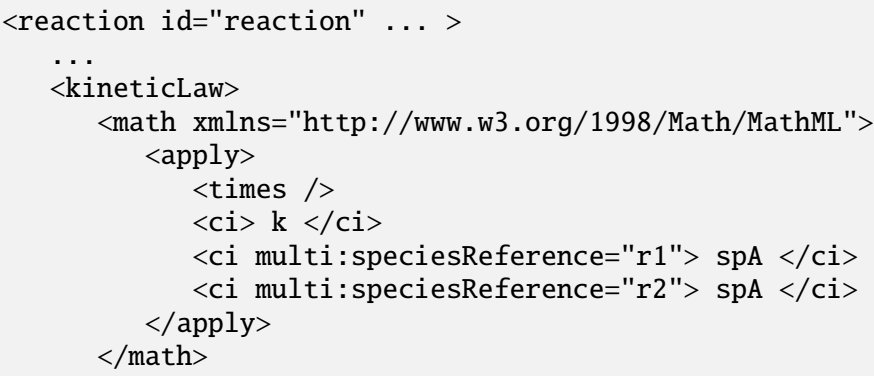




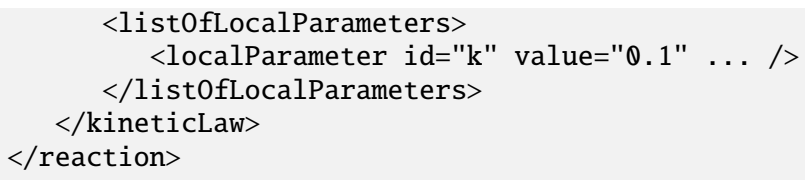

Two "spA" species are distinguished by the "r1" and "r2" speciesReferences respectively.

\subsubsection{The representationType attribute}

The optional representationType attribute, of type RepresentationType, can only be used when the content of the ci element is a species' id or a possibleSpeciesFeatureValue's id. The representationType and speciesReference attributes can both be used for the same ci element at the same time.

The representationType attribute can only have the value of "sum" when the content of the ci is the id of a species. The interpretation of such a ci element is the total concentration or amount of all fully defined species (see Section 3.19 on page 29) mapping to the referenced pattern species.

The representationType attribute can have the value of numericValue when the content of the ci is the id of a possibleSpeciesFeatureValue and the speciesReference attribute must be defined. The interpretation of such a $\mathrm{ci}$ is the same as a ci element having a parameter which the possibleSpeciesFeatureValue links via its numericValue attribute.

The following example demonstrates the use of this attribute for "sum" of species concentrations.

$$
\mathrm{k} 1 * \mathrm{Si} /(\mathrm{k} 2+\mathrm{SUM}(\mathrm{Si}))
$$

In this example, the reactant "Si" is a pattern species which may have multiple fully defined species mapping to it, for example species "S1", "S2", ..., "Sn". "SUM(Si)" is a function to calculate the total concentration of all fully defined species mapping to "Si". The product can be another pattern species "Pi".

The SBML code for the math expression can be as follows:

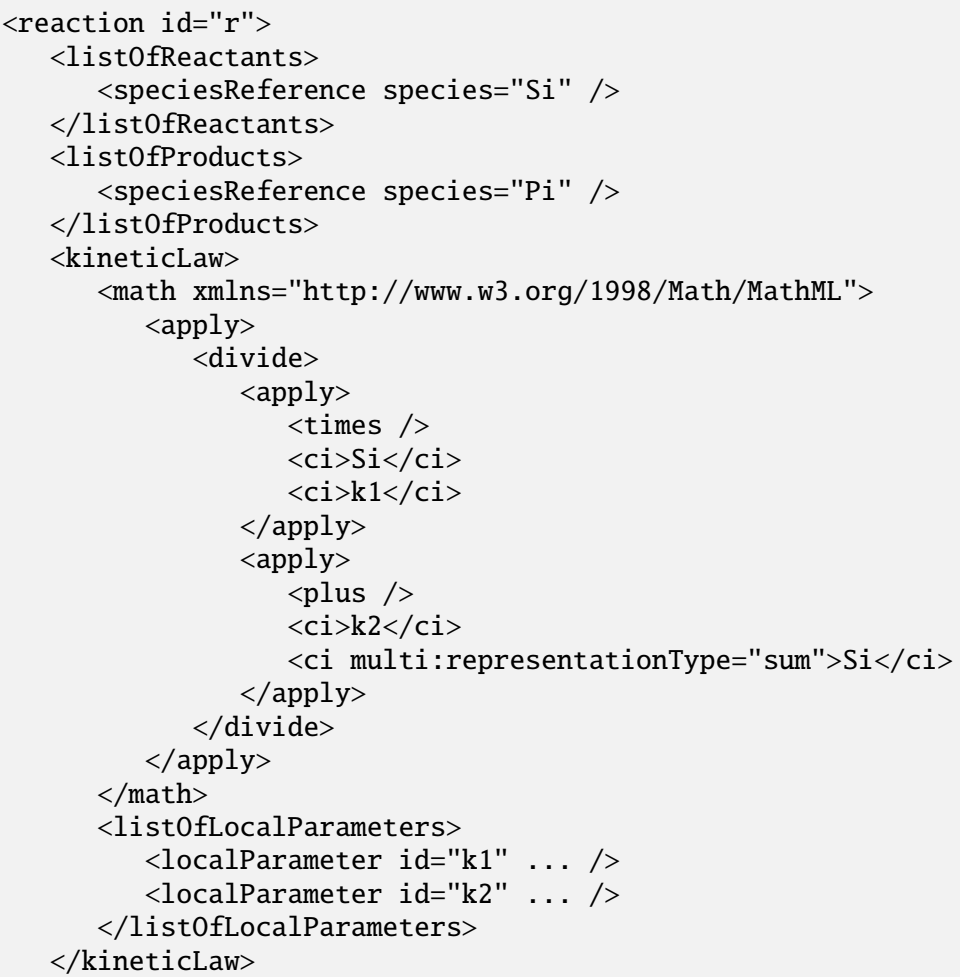


$</$ reaction $>$

The math expressions for the individual species in the example can be:

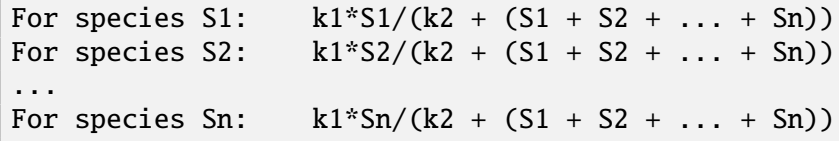

\subsection{Namespace scoping rules for identifiers}

In the Multi package, as in SBML Level 3 Version 1 Core, the Model object contains the main components of an SBML model, such as species, compartments and reactions. The package defines new classes within a model and the scope of the identifiers of those new classes should be defined to prevent identifier collisions. In this section, we describe the scoping rules for all of the types and classes defined in Section 3.3 to Section 3.26 on pages 10-33.

1. The namespace for SId identifiers defined within a Model object used in the Multi package follows the same rules as those defined in SBML Level 3 Version 1 Core for plain Model objects. The scope of the identifiers is limited to the enclosing Model object.

2. The identifier of every SpeciesType and PossibleSpeciesFeatureValue object defined in the Multi package must be unique across the set of all identifiers in the Model object in which it is located.

3. The identifier of every SpeciesTypelnstance, SpeciesTypeComponentIndex, InSpeciesTypeBond and SpeciesFeatureType object defined in the Multi package must be unique across the set of all identifiers of the same class under the direct parent SpeciesType object in which it is located.

4. The identifier of every SpeciesFeature and SubListOfSpeciesFeatures object defined in the Multi package must be unique across the set of all identifiers in the Species object in which it is located.

5. The identifier, if defined, of every CompartmentReference object defined in the Multi package must be unique across the set of all identifiers in the Compartment object in which it is located. 


\section{Examples}

This section contains examples employing the Multi package for SBML Level 3.

\subsection{Example: Compartment, SpeciesType, and Species}

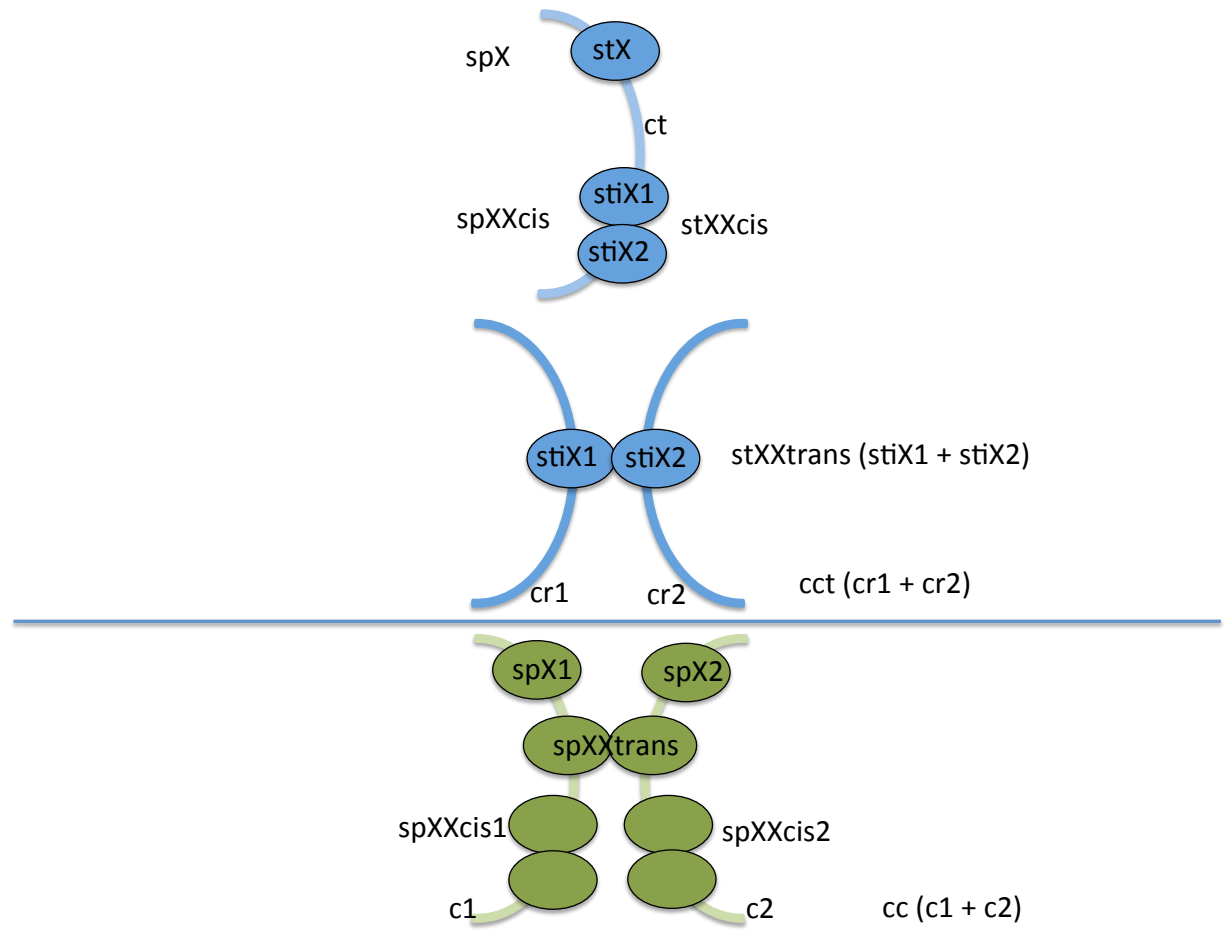

Figure 17: Diagram for an example of Compartment, SpeciesType and Species

Figure 17 shows an example illustrating the usages of and relations among the Compartment, SpeciesType and Species classes.

"ct" is a compartment type. "cct" is a composite compartment type with two compartmentReferences "cr1" and "cr2" both referencing "ct". "c1" is a "not-a-type" compartment and references "ct". Similarly, "c2" is also a "not-a-type" compartment and references "ct". "cc" is a composite "not-a-type" compartment composed of "c1" and "c2".

"stX" is a speciesType on the "ct" compartment. "stXXcis" is a speciesType on the "ct" compartment, and has two speciesTypeInstances "stiX1" and "stX2" both of that reference the "stX" speciesType. "stXXtrans" is a speciesType on the "cct" compartment with two speciesTypeInstances "stiX1" and "stiX2" sitting in different sub-compartments.

"spX" is a species referencing speciesType "stX". "spXXcis" is a species referencing "stXXcis". "spX1" is a species referencing "stX" and sitting in the "c1" compartment. "spX2" is a species also referencing "stX", but sitting in "c2". "spXXtrans" is a species referencing "stXXtrans". "spXXcis1" is a species referencing "stXXtrans" and sitting in "c1"."spXXcis1" is a species referencing "stXXtrans" and sitting in "c2". 
“spX1", "spX2", "spXXtrans”, “spXXcis1" and "spXXcis2” are fully defined species (see Section 3.19 on page 29).

The SBML code can be as follows:

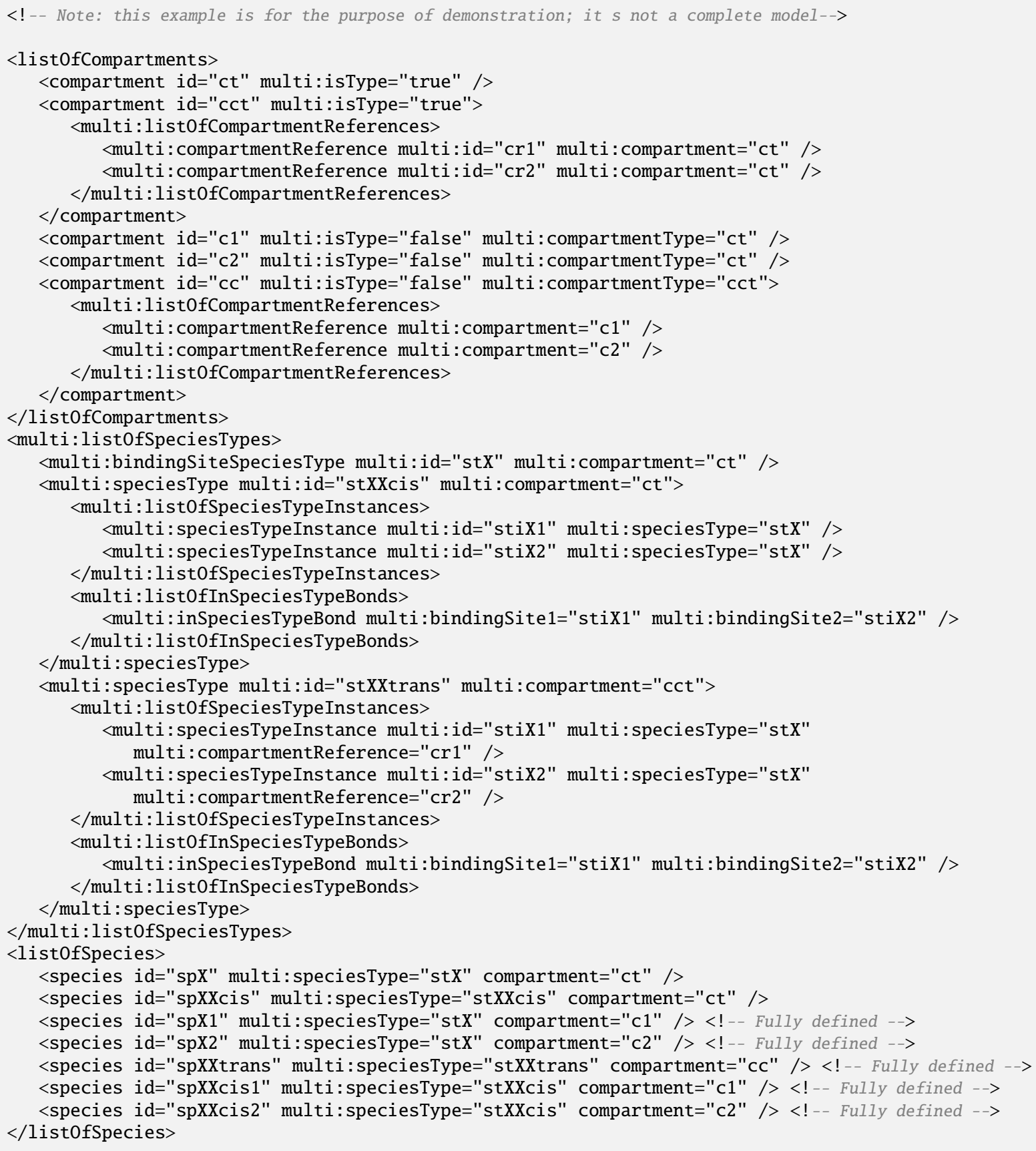

\subsection{Simmune example: the Ecad model}

The Simmune toolset (http: //go . usa.gov/QeH) has some example models including the published Ecad model [Angermann et al. (2012)]. The Ecad model describes the interactions between E-cadherin receptors that can associate either with other E-cadherin receptors within the same membrane (in "cis") or with E-cadherin receptors on adjacent membranes (in "trans"). This model is transformed into the SBML Level 3 format with use of the Multi package. 
$<$ ?xml version="1.0" encoding="UTF-8"?>

<sbml xmlns="http://www.sbml.org/sbml/level3/version1/core" level="3" version="1" xmlns:multi="http://www.sbml.org/sbml/level3/version1/multi/version1" multi:required="true">

<!-- Note: this model has been validated against multi validation rules without errors -->

$<$ model name="E-cadherin_mediated_adhesion">

$<!--$ Definitions -->

$<$ list0fUnitDefinitions $>$

<unitDefinition id="litre_per_mole_per_sec">

$<$ list0fUnits $>$

<unit kind="litre" exponent="1" scale="0" multiplier="1" />

<unit kind="mole" exponent="-1" scale="®" multiplier="1" />

<unit kind="second" exponent="-1" scale="0" multiplier="1" />

$</$ list0fUnits $>$

$</$ unitDefinition $>$

<unitDefinition id="micron_square_per_sec">

$<$ list0fUnits $>$

<unit kind="metre" exponent="2" scale="-6" multiplier="1" />

<unit kind="second" exponent="-1" scale="0" multiplier="1" />

$</$ list0fUnits $>$

$</$ unitDefinition $>$

<unitDefinition id="micrometre_per_sec">

$<$ listOfUnits $>$

<unit kind="metre" exponent="1" scale="-6" multiplier="1" />

<unit kind="second" exponent="-1" scale="0" multiplier="1" />

$</$ list0fUnits $>$

$</$ unitDefinition $>$

<unitDefinition id="per_sec">

$<$ list0fUnits $>$

<unit kind="second" exponent="-1" scale="0" multiplier="1" />

$</$ list0fUnits $>$

$</$ unitDefinition $>$

$</$ list0fUnitDefinitions $>$

$<!--$ Compartments -->

$<$ list0fCompartments $>$

<compartment id="membrane" constant="true" multi:isType="true" />

<compartment id="inter_membrane" constant="true" multi:isType="true"> $<$ multi:list0fCompartmentReferences $>$

<multi:compartmentReference multi:id="m1" multi:compartment="membrane" $/>$ <multi:compartmentReference multi:id="m2" multi:compartment="membrane" $/>$ $</$ multi:list0fCompartmentReferences $>$

$</$ compartment $>$

$</$ list0fCompartments $>$

$<!--$ SpeciesTypes -->

$<$ multi:list0fSpeciesTypes $>$

$<$ !-- Ecad with cis-binding site and trans-binding site: -->

<multi:bindingSiteSpeciesType multi:id="st_Cis_Interface" />

<multi:bindingSiteSpeciesType multi:id="st_Trans_Interface" />

<multi:speciesType multi:id="st_Ecad" multi:compartment="membrane">

$<$ multi:list0fSpeciesTypeInstances>

<multi:speciesTypeInstance multi:id="cis" multi:speciesType="st_Cis_Interface" /> <multi:speciesTypeInstance multi:id="trans" multi:speciesType="st_Trans_Interface" /> $<$ /multi:list0fSpeciesTypeInstances $>$

$</$ multi:speciesType $>$

$<$ !-- cis dimer: -->

<multi:speciesType multi:id="st_Ecad_cis_dimer" multi:compartment="membrane"> $<$ multi:list0fSpeciesTypeInstances>

<multi:speciesTypeInstance multi:id="Ecad1" multi:speciesType="st_Ecad" /> 
$<$ multi:speciesTypeInstance multi:id="Ecad_2" multi:speciesType="st_Ecad" /> $</$ multi:list0fSpeciesTypeInstances $>$

$<$ multi:list0fSpeciesTypeComponentIndexes>

$<$ multi:speciesTypeComponentIndex multi:id="Ecad1cis"

multi:component="cis" multi:identifyingParent="Ecad1" />

$<$ multi:speciesTypeComponentIndex multi:id="Ecad2cis"

multi:component="cis" multi:identifyingParent="Ecad2" />

$<$ multi:speciesTypeComponentIndex multi:id="Ecad1trans"

multi:component="trans" multi:identifyingParent="Ecad1" />

$<$ multi:speciesTypeComponentIndex multi:id="Ecad2trans"

multi:component="trans" multi:identifyingParent="Ecad2" />

$</$ multi:list0fSpeciesTypeComponentIndexes $>$

$<$ multi:list0fInSpeciesTypeBonds $>$

$<$ multi:inSpeciesTypeBond multi:bindingSite1="Ecad1cis"

multi:bindingSite2="Ecad2cis" />

$</$ multi:list0fInSpeciesTypeBonds $>$

$</$ multi:speciesType $>$

$<$ !-- trans dimer: -->

$<$ multi:speciesType multi:id="st_Ecad_trans_dimer" multi:compartment="inter_membrane">

$<$ multi:list0fSpeciesTypeInstances>

$<$ multi:speciesTypeInstance multi:id="Ecad1" multi:speciesType="st_Ecad" multi: compartmentReference="m1" />

$<$ multi:speciesTypeInstance multi:id="Ecad2" multi:speciesType="st_Ecad" multi: compartmentReference="m2" />

$<$ /multi:list0fSpeciesTypeInstances $>$

$<$ multi:list0fSpeciesTypeComponentIndexes>

$<$ multi:speciesTypeComponentIndex multi:id="Ecad1trans"

multi:component="trans" multi:identifyingParent="Ecad1" />

$<$ multi:speciesTypeComponentIndex multi:id="Ecad2trans"

multi:component="trans" multi:identifyingParent="Ecad2" />

<multi:speciesTypeComponentIndex multi:id="Ecad1cis"

multi:component="cis" multi:identifyingParent="Ecad1" />

$<$ multi:speciesTypeComponentIndex multi:id="Ecad2cis"

multi:component="cis" multi:identifyingParent="Ecad2" />

$</$ multi:list0fSpeciesTypeComponentIndexes $>$

$<$ multi:list0fInSpeciesTypeBonds>

$<$ multi:inSpeciesTypeBond multi:bindingSite1="Ecad1trans"

multi:bindingSite2="Ecad2trans" />

$<$ /multi:list0fInSpeciesTypeBonds $>$

$</$ multi:speciesType $>$

$<$ !-- trimer: -->

$<$ multi:speciesType multi:id="st_Ecad_trimer" multi:compartment="inter_membrane">

$<$ multi:list0fSpeciesTypeInstances>

$<$ multi:speciesTypeInstance multi:id="Ecad1" multi:speciesType="st_Ecad" multi: compartmentReference="m1" />

$<$ multi:speciesTypeInstance multi:id="Ecad2" multi:speciesType="st_Ecad" multi: compartmentReference="m1" />

$<$ multi:speciesTypeInstance multi:id="Ecad3" multi:speciesType="st_Ecad" multi: compartmentReference="m2" />

$</$ multi:list0fSpeciesTypeInstances $>$

$<$ multi:list0fSpeciesTypeComponentIndexes>

$<$ multi:speciesTypeComponentIndex multi:id="Ecad1cis"

multi:component="cis" multi:identifyingParent="Ecad1" />

$<$ multi:speciesTypeComponentIndex multi:id="Ecad1trans"

multi:component="trans" multi:identifyingParent="Ecad1" />

$<$ multi:speciesTypeComponentIndex multi:id="Ecad2cis" multi:component="cis" multi:identifyingParent="Ecad2" />

$<$ multi:speciesTypeComponentIndex multi:id="Ecad2trans" multi:component="trans" multi:identifyingParent="Ecad2" />

$<$ multi:speciesTypeComponentIndex multi:id="Ecad3cis"

multi:component="cis" multi:identifyingParent="Ecad3" />

$<$ multi:speciesTypeComponentIndex multi:id="Ecad3trans"

multi:component="trans" multi:identifyingParent="Ecad3" />

$</$ multi:list0fSpeciesTypeComponentIndexes $>$ 
$<$ multi:list0fInSpeciesTypeBonds>

$<$ multi:inSpeciesTypeBond multi:bindingSite1="Ecad1cis"

multi:bindingSite2="Ecad2cis" />

$<$ multi:inSpeciesTypeBond multi:bindingSite1="Ecad1trans"

multi:bindingSite2="Ecad3trans" />

$</$ multi:list0fInSpeciesTypeBonds $>$

$</$ multi:speciesType $>$

$</$ multi:list0fSpeciesTypes $>$

$<!--$ Species -->

$<$ list0fSpecies $>$

$<!--$ free Ecad -->

<species id="sp_Ecad_unbound" name="Ecad_unbound" compartment="membrane" hasOnlySubstanceUnits="false" boundaryCondition="false" constant="false" multi : speciesType="st_Ecad">

$<$ multi:list0fOutwardBindingSites>

$<$ multi: outwardBindingSite multi:component="cis"

multi:bindingStatus="unbound" />

$<$ multi: outwardBindingSite multi:component="trans"

multi:bindingStatus="unbound" />

$<$ /multi:list0fOutwardBindingSites $>$

$</$ species $>$

$<$ !-- Pattern species: Ecad trans unbnd -->

<species id="sp_Ecad_trans_unbnd" name="Ecad_trans_unbnd" compartment="membrane"

has0nlySubstanceUnits="false" boundaryCondition="false" constant="false" multi: speciesType="st_Ecad">

$<$ multi:list0fOutwardBindingSites>

$<$ multi: outwardBindingSite multi:component="trans"

multi:bindingStatus="unbound" />

$<$ /multi:list0fOutwardBindingSites $>$

$</$ species $>$

$<$ !-- Pattern species: Ecad trans bnd -->

<species id="sp_Ecad_trans_bnd" name="Ecad_trans_bnd" compartment="membrane" has0nlySubstanceUnits="false" boundaryCondition="false" constant="false" multi: speciesType="st_Ecad"> $<$ multi:list0fOutwardBindingSites>

$<$ multi: outwardBindingSite multi: component="trans"

multi:bindingStatus="bound" />

$<$ /multi:list0fOutwardBindingSites $>$

$</$ species $>$

$<$ !-- Pattern species: Ecad all -->

<species id="sp_Ecad_all" name="Ecad_all" compartment="membrane"

hasOnlySubstanceUnits="false"

boundaryCondition="false" constant="false" multi: speciesType="st_Ecad" />

$<$ !-- Pattern species: Ecad cis unbnd -->

<species id="sp_Ecad_cis_unbnd" name="Ecad_cis_unbnd" compartment="membrane" has0nlySubstanceUnits="false" boundaryCondition="false" constant="false" multi:speciesType="st_Ecad"> $<$ multi:list0fOutwardBindingSites>

$<$ multi: outwardBindingSite multi:component="cis"

multi:bindingStatus="unbound" />

$</$ multi:list0fOutwardBindingSites $>$

$</$ species $>$

$<$ !-- Pattern species: Ecad cis unbnd, trans bnd -->

<species id="sp_Ecad_6" name="Ecad_6" compartment="membrane" hasOnlySubstanceUnits="false" boundaryCondition="false" constant="false" multi: speciesType="st_Ecad"> $<$ multi:list0fOutwardBindingSites>

$<$ multi: outwardBindingSite multi:component="cis"

multi:bindingStatus="unbound" />

$<$ multi: outwardBindingSite multi:component="trans" 
multi:bindingStatus="bound" /> $<$ /multi:list0fOutwardBindingSites $>$

$</$ species $>$

$<$ !-- Pattern species: Ecad cis bnd, trans unbnd -->

<species id="sp_Ecad_7" name="Ecad_7" compartment="membrane" hasOnlySubstanceUnits="false" boundaryCondition="false" constant="false" multi: speciesType="st_Ecad"> $<$ multi:list0fOutwardBindingSites>

$<$ multi: outwardBindingSite multi:component="cis"

multi:bindingStatus="bound" />

$<$ multi: outwardBindingSite multi:component="trans"

multi:bindingStatus="unbound" />

$<$ /multi:list0fOutwardBindingSites $>$

$</$ species $>$

$<$ !-- Pattern species: Ecad cis dimer -->

<species id="sp_Ecad_cis_dimer" name="Ecad_cis_dimer" compartment="membrane" hasOnlySubstanceUnits="false" boundaryCondition="false" constant="false" multi:speciesType="st_Ecad_cis_dimer" />

<!-- Pattern species: Ecad cis dimer: all trans bnd -->

<species id="sp_EcadEcad_2" name="Ecad.Ecad_2" compartment="membrane"

hasOnlySubstanceUnits="false" boundaryCondition="false" constant="false"

multi:speciesType="st_Ecad_cis_dimer">

$<$ multi:list0fOutwardBindingSites>

$<$ multi: outwardBindingSite multi: component="Ecad1trans"

multi:bindingStatus="bound" />

$<$ multi:outwardBindingSite multi:component="Ecad2trans"

multi:bindingStatus="bound" />

$<$ /multi:list0fOutwardBindingSites $>$

$</$ species $>$

$<$ !-- Pattern species: Ecad trans dimer ->

<species id="sp_EcadEcad_1" name="Ecad.Ecad_1" compartment="inter_membrane" hasonlySubstanceUnits="false" boundaryCondition="false" constant="false" multi:speciesType="st_Ecad_trans_dimer" />

<!-- Pattern species: Ecad trans dimer: all cis bnd -->

<species id="sp_Ecad_trans_dimer_2" name="Ecad_trans_dimer_2" compartment="inter_membrane" hasOnlySubstanceUnits="false" boundaryCondition="false" constant="false" multi:speciesType="st_Ecad_trans_dimer">

$<$ multi:list0fOutwardBindingSites>

$<$ multi: outwardBindingSite multi:component="Ecad1cis"

multi:bindingStatus="bound" />

$<$ multi: outwardBindingSite multi:component="Ecad2cis"

multi : bindingStatus="bound" />

$<$ multi:list0fOutwardBindingSites $>$

$</$ species $>$

$<$ !-- Pattern species: Ecad True Trimer -->

<species id="sp_Ecad_True_Trimer" compartment="inter_membrane" has0nlySubstanceUnits="false" boundaryCondition="false" constant="false" multi:speciesType="st_Ecad_trimer"> $<$ multi:list0fOutwardBindingSites>

$<$ multi: outwardBindingSite multi:component="Ecad2trans"

multi:bindingStatus="unbound" />

$<$ multi: outwardBindingSite multi:component="Ecad3cis"

multi:bindingStatus="unbound" />

$<$ /multi:list0fOutwardBindingSites $>$

$<$ species $>$

$<$ !-- Pattern species: Ecad All Trimer -->

<species id="sp_Ecad_All_Trimer" compartment="inter_membrane" hasonlySubstanceUnits="false" boundaryCondition="false" constant="false" multi:speciesType="st_Ecad_trimer" /> $</$ listOfSpecies $>$

$<!--$ Reactions $-->$ 


\section{$<$ list0fReactions $>$}

$<$ !-- cis association: -->

$<$ reaction id="rc_Cis_Association" name="Cis_Association" reversible="false" fast="false" compartment="membrane">

$<$ list0fReactants $>$

<speciesReference id="Cis_Association_r1" species="sp_Ecad_6" stoichiometry="1" constant="false" />

<speciesReference id="Cis_Association_r2" species="sp_Ecad_6" stoichiometry="1" constant="false" />

$<$ list0fReactants $>$

$<$ list0fProducts $>$

<speciesReference species="sp_EcadEcad_2" constant="false" />

$</$ list0fProducts $>$

$<$ kineticLaw $>$

<math xmlns="http://www.w3.org/1998/Math/MathML">

$<$ apply>

$<$ times $/>$

$<$ ci $>$ kon $</$ ci $>$

$<$ ci multi:speciesReference="Cis_Association_r1" $>$ sp_Ecad_6 $</$ ci $>$

$<$ ci multi:speciesReference="Cis_Association_r2" $>$ sp_Ecad_6 </ci $>$ $</$ apply $>$

$</$ math $>$

$<$ list0fLocalParameters $>$

$<$ localParameter id="kon" value="9000" units="litre_per_mole_per_sec" />

$<$ list0fLocalParameters $>$

$</$ kineticLaw $>$

$</$ reaction $>$

$<$ !-- In species cis association: Here the model requires that the two interacting molecules

are part of one connected complex already prior to the association. Since the necessary

connectivity can only be mediated by the trans binding sites here, these sites must be

bound to the subcomplex (not shown) linking the two interacting molecules.

$-->$

<multi:intraSpeciesReaction id="rc_Intra_Complex_Cis_Association"

name="Intra-Complex_Cis_Association"

reversible="false" fast="false" compartment="membrane">

$<$ list0fReactants>

<speciesReference id="Intra_Complex_Cis_Association_r1" species="sp_Ecad_6" stoichiometry="1" constant="false" />

<speciesReference id="Intra_Complex_Cis_Association_r2" species="sp_Ecad_6" stoichiometry="1" constant="false" />

$</$ list0fReactants $>$

$<$ list0fProducts $>$

<speciesReference species="sp_EcadEcad_2" constant="false" />

$</$ list0fProducts $>$

$<$ kineticLaw $>$

<math xmlns="http://www.w3.org/1998/Math/MathML">

$<$ apply $>$

$<$ times $/>$

$<$ ci $>$ kon $</$ ci $>$

$<$ ci multi:speciesReference="Intra_Complex_Cis_Association_r1"> sp_Ecad_6 </ci> $</$ apply $>$

$</$ math $>$

$<$ list0fLocalParameters $>$

$<$ localParameter id="kon" value="100" units="per_sec" />

$</$ list0fLocalParameters $>$

$</$ kineticLaw $>$

$<$ /multi:intraSpeciesReaction $>$

$<$ !-- trans association: -->

$<$ reaction id="rc_Trans_Association" name="Trans_Association" reversible="false" fast="false" compartment="inter_membrane">

$<$ listofReactants $>$

<speciesReference id="Trans_Association_r1" species="sp_Ecad_trans_unbnd"

multi: compartmentReference="m1" constant="false" /> 


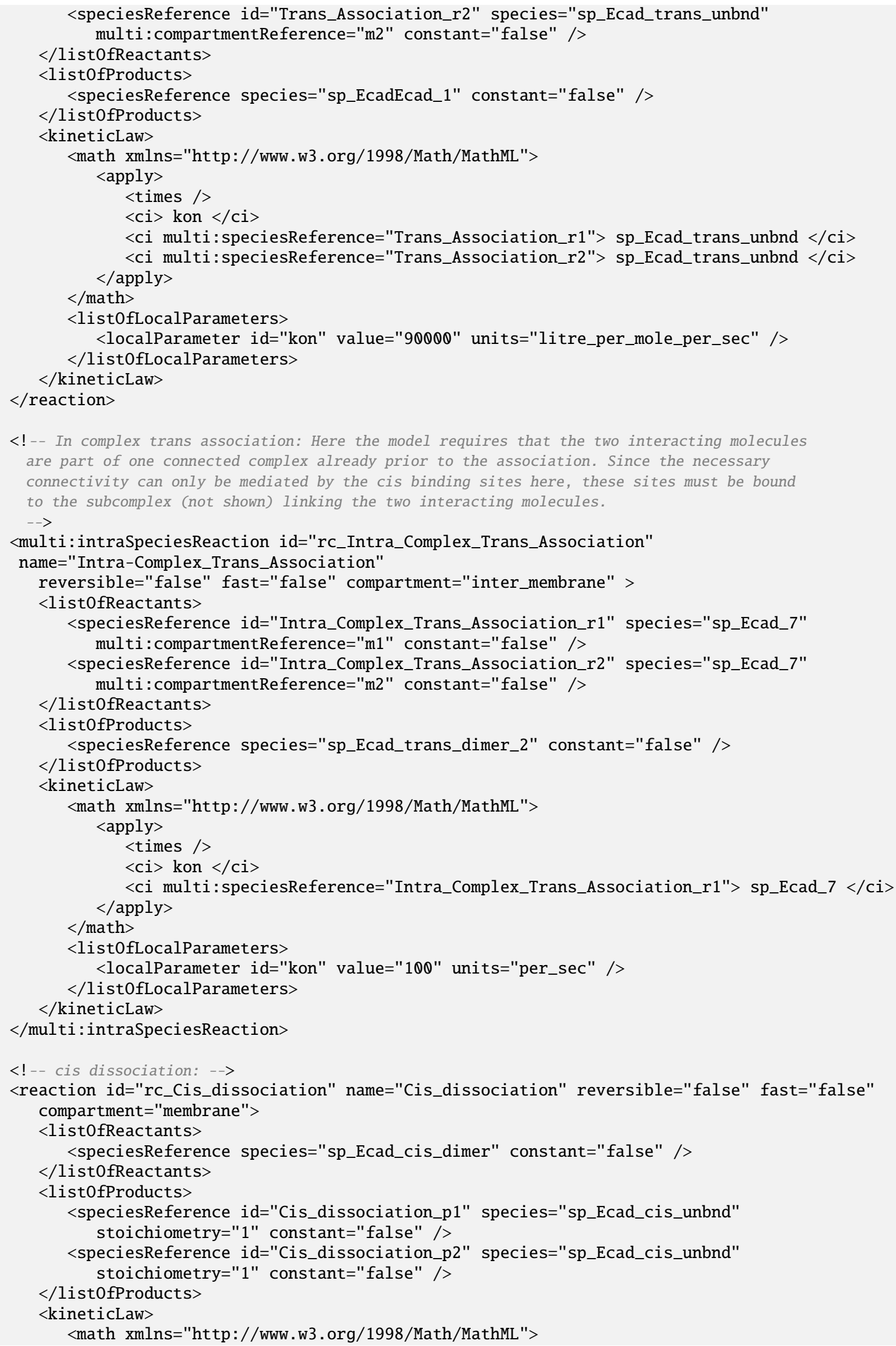




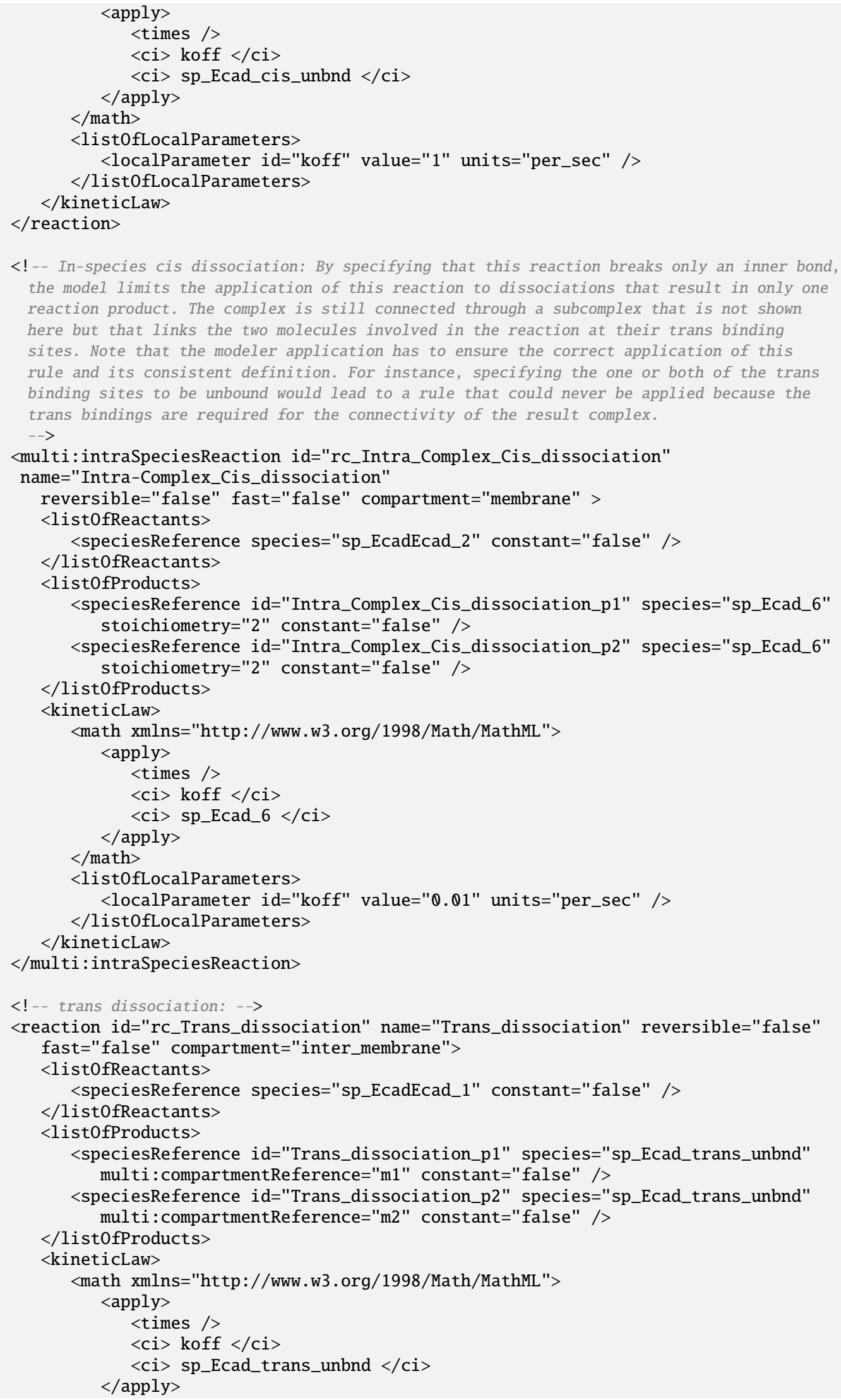




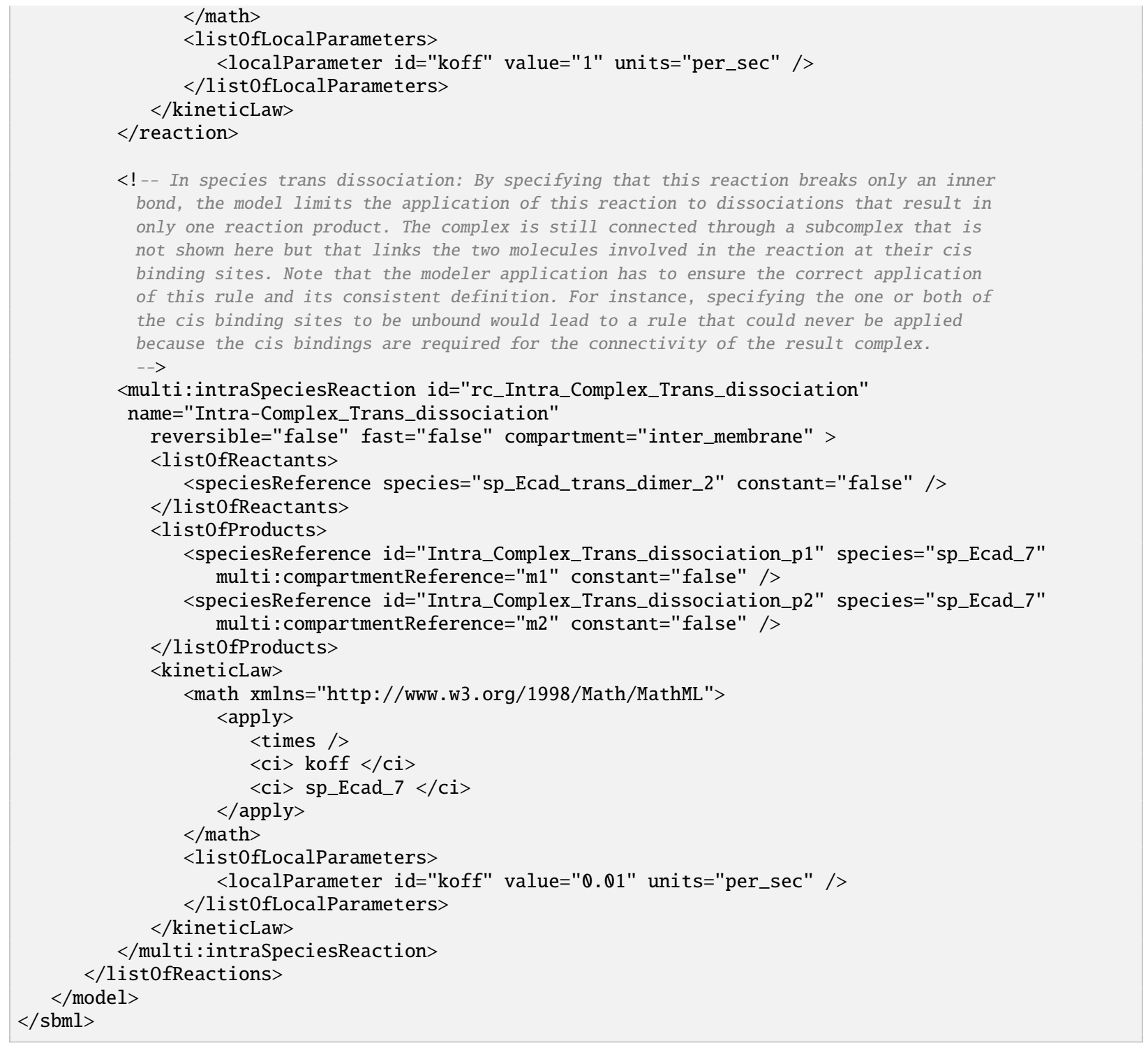

\subsection{A BioNetGen example from its user manual}

egfr_simple.bngl (http: //bionetgen.org/index .php/BNGManual :Listing_1)

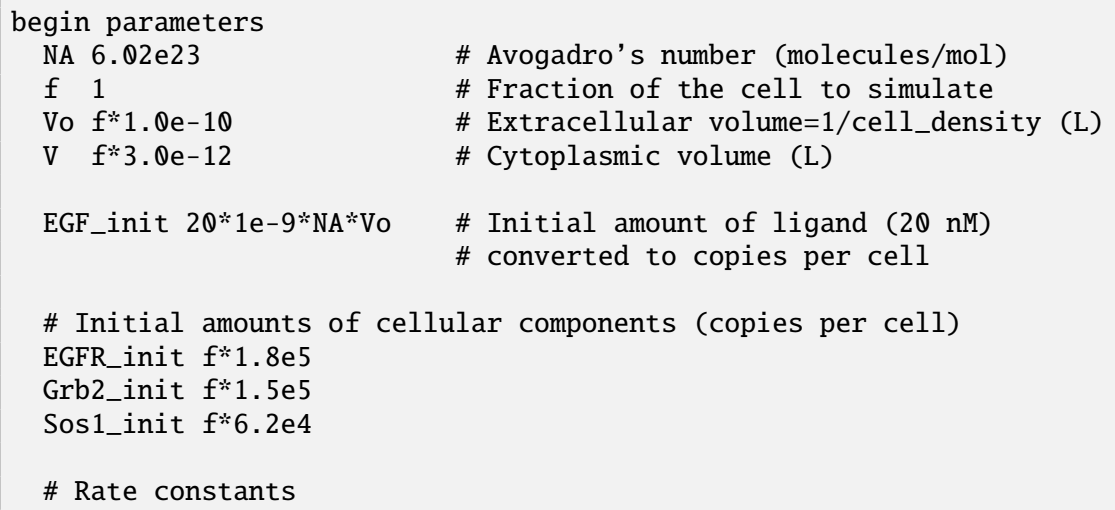




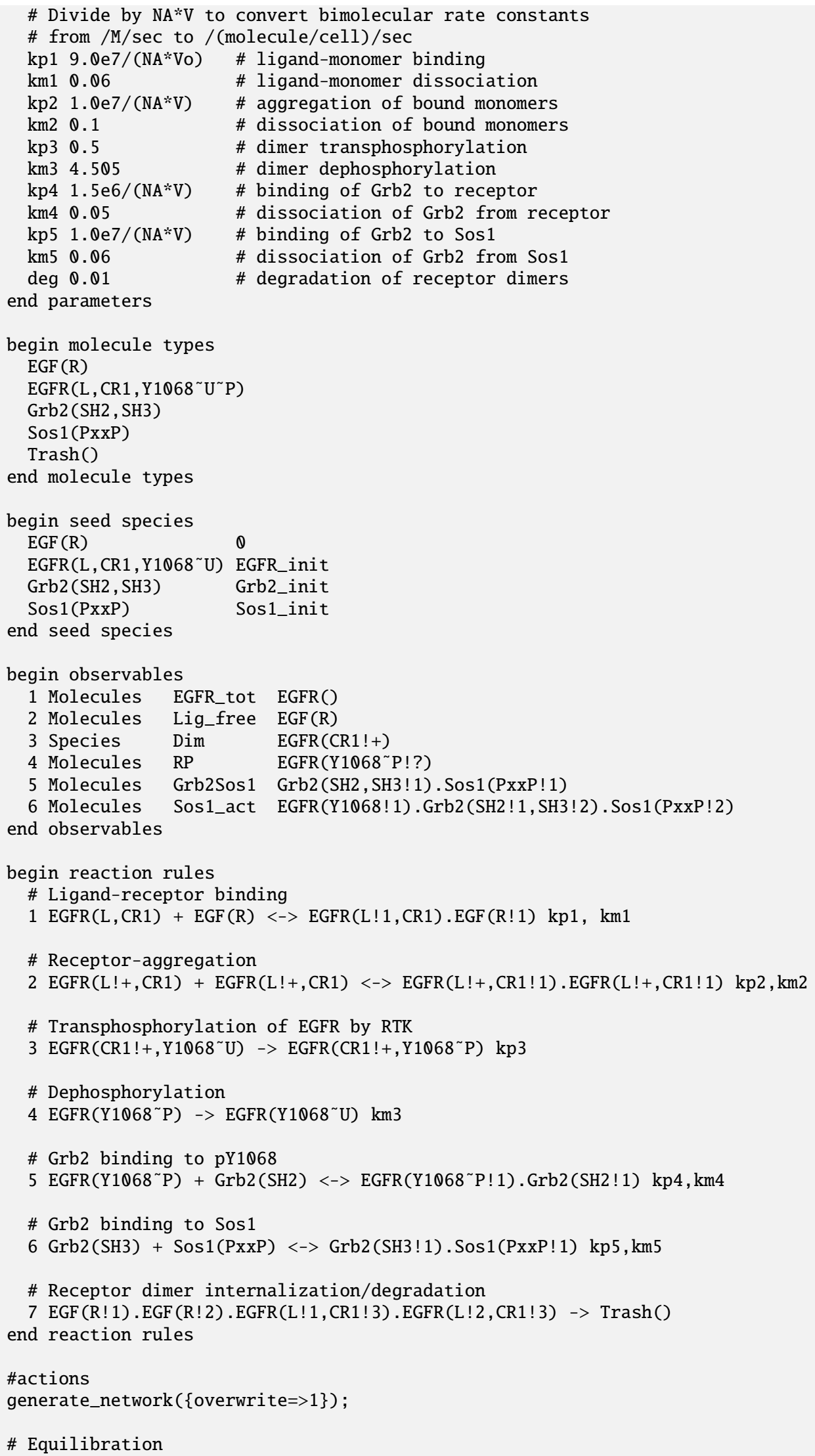


simulate_ode ( $\{$ suffix=>equil,t_end $=>100000$, n_steps $=>10$, sparse $=>1$, steady_state $=>1\}$ ); setConcentration("EGF(R)", "EGF_init"); saveConcentrations(); \# Saves concentrations for future reset

\section{\# Kinetics} writeSBML $(\{\})$;

simulate_ode $(\{t$ t_end $=>120$, n_steps $=>120\})$;

resetConcentrations(); \# reverts to saved Concentrations

simulate_ssa $(\{$ suffix $=>$ ssa,t_end $=>120$, n_steps $=>120\})$;

The SBML code can be as follows. Please note, the SBML code does not cover the content other than the model in the bngl file, such as the "actions", "Equilibration" and "Kinetics" sections.

$<$ ?xml version="1.0" encoding="UTF-8"?>

<sbml xmlns="http://www.sbml.org/sbml/level3/version1/core" level="3" version="1"

xmlns:multi="http://www.sbml.org/sbml/level3/version1/multi/version1" multi:required="true">

$<!--$ Note: this model has been validated agains multi validation rules without errors -->

$<$ model name="bionetgen_example_egfr_simple">

$<$ list0fUnitDefinitions $>$

<unitDefinition id="molecules_per_mol">

$<$ list0fUnits $>$

<unit kind="mole" scale="0" multiplier="1" exponent="-1" /> $</$ listofUnits $>$

$</$ unitDefinition $>$

$</$ list0fUnitDefinitions $>$

$<!--$ compartments -->

$<$ list0fCompartments $>$

<compartment id="Vo" constant="true" spatialDimensions="3" units="litre" multi:isType="false" />

<compartment $i d=" V "$ constant="true" spatialDimensions="3" units="litre" multi:isType="false" />

$</$ list0fCompartments $>$

$<!--$ speciesType -->

$<$ multi:list0fSpeciesTypes $>$

$<!--E G F(R)->$

$<$ multi:bindingSiteSpeciesType multi:id="st_EGF_bs_R" />

$<$ multi:speciesType multi:id="st_EGF">

$<$ multi:list0fSpeciesTypeInstances>

<multi:speciesTypeInstance multi:id="R" multi:speciesType="st_EGF_bs_R" />

$<$ /multi:list0fSpeciesTypeInstances $>$

$</$ multi:speciesType $>$

$<!--E G F R\left(L, C R 1, Y 1068^{\sim} U^{\sim} P\right)->$

<multi:bindingSiteSpeciesType multi:id="st_EGFR_bs_L" />

$<$ multi:bindingSiteSpeciesType multi:id="st_EGFR_bs_CR1" />

<multi:bindingSiteSpeciesType multi:id="st_EGFR_bs_Y1068">

$<$ multi:list0fSpeciesFeatureTypes>

<multi:speciesFeatureType multi:id="sft_Y1068" multi:occur="1">

$<$ multi:list0fPossibleSpeciesFeatureValues>

$<$ multi:possibleSpeciesFeatureValue multi:id="U" />

<multi:possibleSpeciesFeatureValue multi:id="P" />

$</$ multi:list0fPossibleSpeciesFeatureValues>

$</$ multi:speciesFeatureType $>$

$</$ multi:listOfSpeciesFeatureTypes $>$

$</$ multi:bindingSiteSpeciesType $>$

$<$ multi:speciesType multi:id="st_EGFR">

$<$ multi:list0fSpeciesTypeInstances>

<multi:speciesTypeInstance multi:id="L" multi:speciesType="st_EGFR_bs_L" />

<multi:speciesTypeInstance multi:id="CR1" multi:speciesType="st_EGFR_bs_CR1" /> 
<multi:speciesTypeInstance multi:id="Y1068" multi:speciesType="st_EGFR_bs_Y1068" /> $</$ multi:list0fSpeciesTypeInstances $>$

$</$ multi:speciesType $>$

$<!--E G F R$ dimer: $[E G F R(C R 1 ! 1) . E G F R(C R 1 ! 1)]->$

$<$ multi:speciesType multi:id="st_EGFR_dimer">

$<$ multi:list0fSpeciesTypeInstances>

<multi:speciesTypeInstance multi:id="EGFR1" multi:speciesType="st_EGFR" />

$<$ multi:speciesTypeInstance multi:id="EGFR2" multi:speciesType="st_EGFR" />

$</$ multi:list0fSpeciesTypeInstances $>$

$<$ multi:list0fSpeciesTypeComponentIndexes>

$<$ multi:speciesTypeComponentIndex multi:id="EGFR1CR1"

multi:component="CR1" identifyingParent="EGFR1" />

$<$ multi:speciesTypeComponentIndex multi:id="EGFR2CR1"

multi:component="CR1" identifyingParent="EGFR2" />

$</$ multi:list0fSpeciesTypeComponentIndexes $>$

$<$ multi:list0fInSpeciesTypeBonds>

$<$ multi:inSpeciesTypeBond multi:bindingSite1="EGFR1CR1" multi:bindingSite2="EGFR2CR1" />

$</$ multi:list0fInSpeciesTypeBonds $>$

$</$ multi:speciesType $>$

$<$ !-- EGFR-EGF dimer: [EGF(R!1).EGF(R!2).EGFR(L!1,CR1!3).EGFR(L!2,CR1!3)] -->

$<$ multi:speciesType multi:id="st_EGFR_EGF_dimer">

$<$ multi:list0fSpeciesTypeInstances>

$<$ multi:speciesTypeInstance multi:id="EGF1" multi:speciesType="st_EGF" />

$<$ multi:speciesTypeInstance multi:id="EGF2" multi:speciesType="st_EGF" />

<multi:speciesTypeInstance multi:id="EGFR1" multi:speciesType="st_EGFR" />

$<$ multi:speciesTypeInstance multi:id="EGFR2" multi:speciesType="st_EGFR" />

$</$ multi:list0fSpeciesTypeInstances $>$

$<$ multi:list0fSpeciesTypeComponentIndexes>

$<$ multi:speciesTypeComponentIndex multi:id="EGF1R"

multi:component="R" identifyingParent="EGF1" />

$<$ multi:speciesTypeComponentIndex multi:id="EGF2R"

multi:component="R" identifyingParent="EGF2" />

$<$ multi: speciesTypeComponentIndex multi:id="EGFR1L"

multi:component="L" identifyingParent="EGFR1" />

$<$ multi:speciesTypeComponentIndex multi:id="EGFR2L"

multi:component="L" identifyingParent="EGFR2" />

$<$ multi:speciesTypeComponentIndex multi:id="EGFR1CR1"

multi:component="CR1" identifyingParent="EGFR1" />

$<$ multi:speciesTypeComponentIndex multi:id="EGFR2CR1"

multi:component="CR1" identifyingParent="EGFR2" />

$</$ multi:list0fSpeciesTypeComponentIndexes $>$

$<$ multi:list0fInSpeciesTypeBonds $>$

<multi:inSpeciesTypeBond multi:bindingSite1="EGFR1CR1" multi:bindingSite2="EGFR2CR1" />

$<$ multi:inSpeciesTypeBond multi:bindingSite1="EGF1R" multi:bindingSite2="EGFR1L" />

$<$ multi:inSpeciesTypeBond multi:bindingSite1="EGF2R" multi:bindingSite2="EGFR2L" />

$<$ multi:list0fInSpeciesTypeBonds $>$

$</$ multi:speciesType $>$

$<!--\operatorname{Grb} 2(\mathrm{SH} 2, \mathrm{SH} 3)-->$

$<$ multi:bindingSiteSpeciesType multi:id="st_Grb2_bs_SH2" />

$<$ multi:bindingSiteSpeciesType multi:id="st_Grb2_bs_SH3" />

$<$ multi:speciesType multi:id="st_Grb2">

$<$ multi:list0fSpeciesTypeInstances>

$<$ multi:speciesTypeInstance multi:id="SH2" multi:speciesType="st_Grb2_bs_SH2" />

<multi:speciesTypeInstance multi:id="SH3" multi:speciesType="st_Grb2_bs_SH3" />

$</$ multi:list0fSpeciesTypeInstances $>$

$</$ multi:speciesType $>$

$<!--$ Sos 1 -->

$<$ multi:bindingSiteSpeciesType multi:id="st_Sos1_bs_PxxP" />

$<$ multi:speciesType multi:id="st_Sos1">

$<$ multi:list0fSpeciesTypeInstances>

<multi:speciesTypeInstance multi:id="PxxP" multi:speciesType="st_Sos1_bs_PxxP" /> 
$</$ multi:list0fSpeciesTypeInstances $>$

$</$ multi:speciesType $>$

$<!--$ Trash - >

$<$ multi:speciesType multi:id="st_trash" />

$<!--$ Grb2-Sos1 -->

<multi:speciesType multi:id="st_Grb2_Sos1">

$<$ multi:list0fSpeciesTypeInstances>

<multi:speciesTypeInstance multi:id="Grb2" multi:speciesType="st_Grb2" />

<multi:speciesTypeInstance multi:id="Sos1" multi:speciesType="st_Sos1" />

$</$ multi:list0fSpeciesTypeInstances $>$

$<$ multi:list0fInSpeciesTypeBonds>

<multi:inSpeciesTypeBond multi:bindingSite1="SH3" multi:bindingSite2="PxxP" />

$<$ /multi:list0fInSpeciesTypeBonds $>$

$</$ multi:speciesType $>$

$<!--\operatorname{EGFR}(Y 1068 ! 1) \cdot \operatorname{Grb1} 1$ SH2!1, SH3!2).Sos1(PXXP!2) - >

$<$ multi:speciesType multi:id="st_EGFR_Grb2_Sos1">

$<$ multi:list0fSpeciesTypeInstances>

<multi:speciesTypeInstance multi:id="EGFR" multi:speciesType="st_EGFR" $/>$

<multi:speciesTypeInstance multi:id="Grb2" multi:speciesType="st_Grb2" />

<multi:speciesTypeInstance multi:id="Sos1" multi:speciesType="st_Sos1" />

$</$ multi:list0fSpeciesTypeInstances $>$

$<$ multi:list0fInSpeciesTypeBonds>

<multi:inSpeciesTypeBond multi:bindingSite1="Y1068" multi:bindingSite2="SH2" /> <multi:inSpeciesTypeBond multi:bindingSite1="SH3" multi:bindingSite2="PxxP" /> $</$ multi:list0fInSpeciesTypeBonds $>$

$</$ multi:speciesType $>$

$<!--E G F R(L ! 1) \cdot E G F(R ! 1)-->$

$<$ multi:speciesType multi:id="st_EGFR_EGF">

$<$ multi:list0fSpeciesTypeInstances>

<multi:speciesTypeInstance multi:id="EGFR" multi:speciesType="st_EGFR" $/>$ <multi:speciesTypeInstance multi:id="EGF" multi:speciesType="st_EGF" />

$<$ /multi:list0fSpeciesTypeInstances $>$

$<$ multi:list0fInSpeciesTypeBonds>

<multi:inSpeciesTypeBond multi:bindingSite1="L" multi:bindingSite2="R" /> $<$ /multi:list0fInSpeciesTypeBonds $>$

$</$ multi:speciesType $>$

$<!--\operatorname{EGFR}(Y 1068 ! 1) \cdot G r b 2(S H 2 ! 1)->$

$<$ multi:speciesType multi:id="st_EGFR_Grb2">

$<$ multi:list0fSpeciesTypeInstances>

<multi:speciesTypeInstance multi:id="EGFR" multi:speciesType="st_EGFR" $/>$

<multi:speciesTypeInstance multi:id="Grb2" multi:speciesType="st_Grb2" />

$</$ multi:list0fSpeciesTypeInstances $>$

$<$ multi:list0fInSpeciesTypeBonds $>$

$<$ multi:inSpeciesTypeBond multi:bindingSite1="Y1068" multi:bindingSite2="SH2" /> $<$ /multi:list0fInSpeciesTypeBonds $>$

$</$ multi:speciesType $>$

$</$ multi:list0fSpeciesTypes $>$

$<!--$ species -->

$<$ list0fSpecies $>$

<species id="sp_EGF_free" name="EGF(R)" compartment="Vo" multi: speciesType="st_EGF" hasOnlySubstanceUnits="false" boundaryCondition="false" constant="false"> $<$ multi:list0fOutwardBindingSites>

<multi:outwardBindingSite multi:component="R" multi:bindingStatus="unbound" /> $</$ multi:list0fOutwardBindingSites $>$

$</$ species $>$

<species id="sp_EGFR_free_U" name="EGFR(L,CR1,Y1068 U) " compartment="V" multi : speciesType="st_EGFR" hasOnlySubstanceUnits="false" 
boundaryCondition="false" constant="false">

$<$ multi:list0fOutwardBindingSites>

<multi:outwardBindingSite multi:component="L" multi:bindingStatus="unbound" />

$<$ multi: outwardBindingSite multi:component="CR1" multi:bindingStatus="unbound" />

<multi: outwardBindingSite multi:component="Y1068" multi:bindingStatus="unbound" />

$</$ multi:list0fOutwardBindingSites $>$

$<$ multi:list0fSpeciesFeatures>

<multi:speciesFeature multi:speciesFeatureType="sft_Y1068" multi:occur="1"> $<$ multi:list0fSpeciesFeatureValues $>$

<multi:speciesFeatureValue multi:value="U" />

$</$ multi:list0fSpeciesFeatureValues $>$

$</$ multi:speciesFeature $>$

$<$ /multi:listOfSpeciesFeatures $>$

$</$ species $>$

<species id="sp_Grb2_free" name="Grb2(SH2,SH3)" compartment="Vo" multi:speciesType="st_Grb2" hasOnlySubstanceUnits="false" boundaryCondition="false" constant="false"> $<$ multi:list0fOutwardBindingSites>

$<$ multi: outwardBindingSite multi:component="SH2" multi:bindingStatus="unbound" /> $<$ multi:outwardBindingSite multi:component="SH3" multi:bindingStatus="unbound" /> $</$ multi:list0fOutwardBindingSites $>$

$</$ species $>$

<species id="sp_Grb2_SH2" name="Grb2(SH2)" compartment="V" multi: speciesType="st_Grb2" hasOnlySubstanceUnits="false"

boundaryCondition="false" constant="false">

$<$ multi:list0fOutwardBindingSites>

<multi: outwardBindingSite multi:component="SH2" multi:bindingStatus="unbound" /> $</$ multi:list0fOutwardBindingSites $>$

$</$ species $>$

<species id="sp_Grb2_SH3" name="Grb2(SH3)" compartment="V" multi:speciesType="st_Grb2" hasOnlySubstanceUnits="false"

boundaryCondition="false" constant="false">

$<$ multi:list0fOutwardBindingSites>

$<$ multi: outwardBindingSite multi:component="SH3" multi:bindingStatus="unbound" /> $</$ multi:listofOutwardBindingSites $>$

$</$ species $>$

<species id="sp_Sos1_free" name="Sos1(PxxP)" compartment="V" multi:speciesType="st_Sos1" hasOnlySubstanceUnits="false" boundaryCondition="false" constant="false"> <multi:list0fOutwardBindingSites>

<multi: outwardBindingSite multi:component="PxxP" multi:bindingStatus="unbound" /> $</$ multi:list0fOutwardBindingSites $>$

$<$ species $>$

<species id="sp_EGF_tot" name="EGF()" compartment="Vo" multi: speciesType="st_EGF" hasOnlySubstanceUnits="false"

boundaryCondition="false" constant="false" />

<species id="sp_EGFR_dimerized" name="EGFR(CR1!+)" compartment="V"

multi: speciesType="st_EGFR" hasOnlySubstanceUnits="false" boundaryCondition="false" constant="false">

$<$ multi:list0fOutwardBindingSites>

<multi:outwardBindingSite multi:component="CR1" multi:bindingStatus="bound" /> $</$ multi:list0fOutwardBindingSites $>$

$</$ species $>$

<species id="sp_EGFR_U" name="EGFR(Y1068 P!?)" compartment="V"

multi: speciesType="st_EGFR" hasOnlySubstanceUnits="false" boundaryCondition="false" constant="false">

$<$ multi:list0fSpeciesFeatures>

<multi:speciesFeature multi:speciesFeatureType="sft_Y1068" multi:occur="1"> $<$ multi:list0fSpeciesFeatureValues>

$<$ multi:speciesFeatureValue multi:value="P" />

$</$ multi:list0fSpeciesFeatureValues $>$

$</$ multi:speciesFeature $>$

$</$ multi:listOfSpeciesFeatures $>$

$</$ species $>$

<species id="sp_EGFR_L_CR1" name="EGFR(L,CR1)" compartment="V" multi:speciesType="st_EGFR" hasOnlySubstanceUnits="false" boundaryCondition="false" constant="false"> <multi:list0fOutwardBindingSites> $<$ multi:outwardBindingSite multi:component="L" multi:bindingStatus="unbound" /> 
$<$ multi: outwardBindingSite multi:component="CR1" multi:bindingStatus="unbound" /> $<$ /multi:list0fOutwardBindingSites $>$

$<$ species $>$

<species id="sp_EGFR_EGF_CR1" name="EGFR(L!1,CR1).EGF(R!1)" compartment="V"

multi: speciesType="st_EGFR_EGF" hasOnlySubstanceUnits="false" boundaryCondition="false" constant="false"> $<$ multi:list0fOutwardBindingSites>

<multi: outwardBindingSite multi:component="CR1" multi:bindingStatus="unbound" /> $<$ multi:list0foutwardBindingSites $>$

$</$ species $>$

<species id="sp_EGFR_bL_CR1" name="EGFR(L!+,CR1)" compartment="V"

multi: speciesType="st_EGFR" hasOnlySubstanceUnits="false" boundaryCondition="false" constant="false">

$<$ multi:list0fOutwardBindingSites>

<multi: outwardBindingSite multi:component="L" multi:bindingStatus="bound" />

<multi:outwardBindingSite multi:component="CR1" multi:bindingStatus="unbound" /> $<$ /multi:list0fOutwardBindingSites $>$

$</$ species $>$

<species id="sp_EGFR_dimer_bL" name="EGFR(L!+,CR1!1).EGFR(L!+,CR1!1)" compartment="V" multi: speciesType="st_EGFR_dimer"

hasOnlySubstanceUnits="false" boundaryCondition="false" constant="false"> $<$ multi:list0fOutwardBindingSites>

$<$ multi: outwardBindingSite multi: component="EGFR1L"

multi:bindingStatus="bound" />

$<$ multi: outwardBindingSite multi:component="EGFR2L"

multi : bindingStatus="bound" />

$</$ multi:list0fOutwardBindingSites $>$

$</$ species $>$

<species id="sp_EGFR_EGF_dimer" name="EGF(R!1).EGF(R!2).EGFR(L!1,CR1!3).EGFR(L!2,CR1!3)" compartment="V" multi:speciesType="st_EGFR_EGF_dimer" hasOnlySubstanceUnits="false" boundaryCondition="false" constant="false" />

<species id="sp_EGFR_bCR1_Y1068_U" name="EGFR(CR1!+,Y1068〜U)" compartment="V"

multi: speciesType="st_EGFR" hasOnlySubstanceUnits="false" boundaryCondition="false" constant="false">

$<$ multi:list0fOutwardBindingSites $>$

$<$ multi: outwardBindingSite multi:component="CR1" multi:bindingStatus="bound" />

$<$ multi: outwardBindingSite multi:component="Y1068" multi:bindingStatus="unbound" />

$</$ multi:list0fOutwardBindingSites $>$

$<$ multi:list0fSpeciesFeatures>

<multi:speciesFeature multi:speciesFeatureType="sft_Y1068" multi:occur="1"> $<$ multi:list0fSpeciesFeatureValues>

$<$ multi:speciesFeatureValue multi:value="U" />

$</$ multi:list0fSpeciesFeatureValues $>$

$</$ multi:speciesFeature $>$

$</$ multi:list0fSpeciesFeatures $>$

$<$ /species $>$

<species id="sp_EGFR_bCR1_Y1068_P" name="EGFR(CR1!+,Y1068〜P)" multi:speciesType="st_EGFR" compartment="V" hasOnlySubstanceUnits="false" boundaryCondition="false" constant="false"> $<$ multi:list0fOutwardBindingSites>

<multi:outwardBindingSite multi:component="CR1" multi:bindingStatus="bound" />

$<$ multi:outwardBindingSite multi:component="Y1068" multi:bindingStatus="unbound" />

$<$ /multi:list0fOutwardBindingSites $>$

$<$ multi:list0fSpeciesFeatures>

<multi:speciesFeature multi:speciesFeatureType="sft_Y1068" multi:occur="1"> $<$ multi:list0fSpeciesFeatureValues>

$<$ multi:speciesFeatureValue multi:value="P" />

$</$ multi:list0fSpeciesFeatureValues $>$

$</$ multi:speciesFeature $>$

$<$ /multi:list0fSpeciesFeatures $>$

$</$ species $>$

<species id="sp_EGFR_Y1068_P" name="EGFR(Y1068 P)" multi : speciesType="st_EGFR"

compartment="V" hasOnlySubstanceUnits="false" boundaryCondition="false" constant="false"> $<$ multi:list0fOutwardBindingSites>

<multi: outwardBindingSite multi:component="Y1068" multi:bindingStatus="unbound" /> $<$ /multi:list0fOutwardBindingSites $>$

$<$ multi:list0fSpeciesFeatures $>$ 
<multi:speciesFeature multi:speciesFeatureType="sft_Y1068" multi:occur="1"> $<$ multi:list0fSpeciesFeatureValues>

$<$ multi:speciesFeatureValue multi:value="P" />

$</$ multi:list0fSpeciesFeatureValues $>$

$</$ multi:speciesFeature $>$

$<$ /multi:list0fSpeciesFeatures $>$

$<$ species $>$

<species id="sp_EGFR_Y1068_U" name="EGFR(Y1068 U)" multi:speciesType="st_EGFR"

compartment="V" hasOnlySubstanceUnits="false" boundaryCondition="false" constant="false"> $<$ multi:list0fOutwardBindingSites>

<multi: outwardBindingSite multi:component="Y1068" multi:bindingStatus="unbound" />

$<$ multi:list0fOutwardBindingSites $>$

$<$ multi:list0fSpeciesFeatures>

<multi:speciesFeature multi:speciesFeatureType="sft_Y1068" multi:occur="1"> $<$ multi:list0fSpeciesFeatureValues $>$

$\langle$ multi:speciesFeatureValue multi:value="U" />

$<$ /multi:list0fSpeciesFeatureValues $>$

$</$ multi:speciesFeature $>$

$</$ multi:list0fSpeciesFeatures $>$

$</$ species $>$

<species id="sp_EGFR_Grb2_P" name="EGFR(Y1068 P!1).Grb2(SH2!1)" compartment="V"

multi: speciesType="st_EGFR_Grb2"

has0nlySubstanceUnits="false" boundaryCondition="false" constant="false"> $<$ multi:list0fSpeciesFeatures>

<multi:speciesFeature multi:speciesFeatureType="sft_Y1068" multi:occur="1"> $<$ multi:list0fSpeciesFeatureValues>

$<$ multi:speciesFeatureValue multi:value="P" />

$</$ multi:list0fSpeciesFeatureValues $>$

$</$ multi:speciesFeature $>$

$</$ multi:list0fSpeciesFeatures $>$

$</$ species $>$

<species id="sp_Grb2_Sos1" name="Grb2(SH3!1).Sos1(PxxP!1)" compartment="V"

multi: speciesType="st_Grb2_Sos1" hasOnlySubstanceUnits="false"

boundaryCondition="false" constant="false" />

<species id="sp_Trash" name="Trash()" compartment="V" multi:speciesType="st_trash" hasOnlySubstanceUnits="false"

boundaryCondition="false" constant="false" />

$</$ listOfSpecies $>$

$<!--$ parameters - $>$

$<$ list0fParameters $>$

$<$ parameter id="NA" value="6.02e23" constant="true" units="molecules_per_mol" />

$<$ parameter id="f" value="1" constant="true" />

$<$ parameter id="kp1" constant="true" />

$<$ parameter id="km1" value="0.06" constant="true" />

$<$ parameter id="kp2" constant="true" />

$<$ parameter id="km2" value="0.1" constant="true" />

$<$ parameter id="kp3" value="0.5" constant="true" />

$<$ parameter id="km3" value="4.505" constant="true" />

$<$ parameter id="kp4" constant="true" />

$<$ parameter id="km4" value="0.05" constant="true" />

$<$ parameter id="kp5" constant="true" />

$<$ parameter id="km5" value="0.06" constant="true" />

$<$ parameter id="deg" value="0.01" constant="true" />

$</$ list0fParameters $>$

$<$ !-- intiialAssignments -->

$<$ list0fInitialAssignments $>$

<initialAssignment symbol="Vo">

<math xmlns="http://www.w3.org/1998/Math/MathML"> $<$ apply>

$<$ times />

$<\mathrm{ci}>\mathrm{f}</ \mathrm{ci}>$

$<$ cn $>1 \mathrm{e}-10</ \mathrm{cn}>$ 


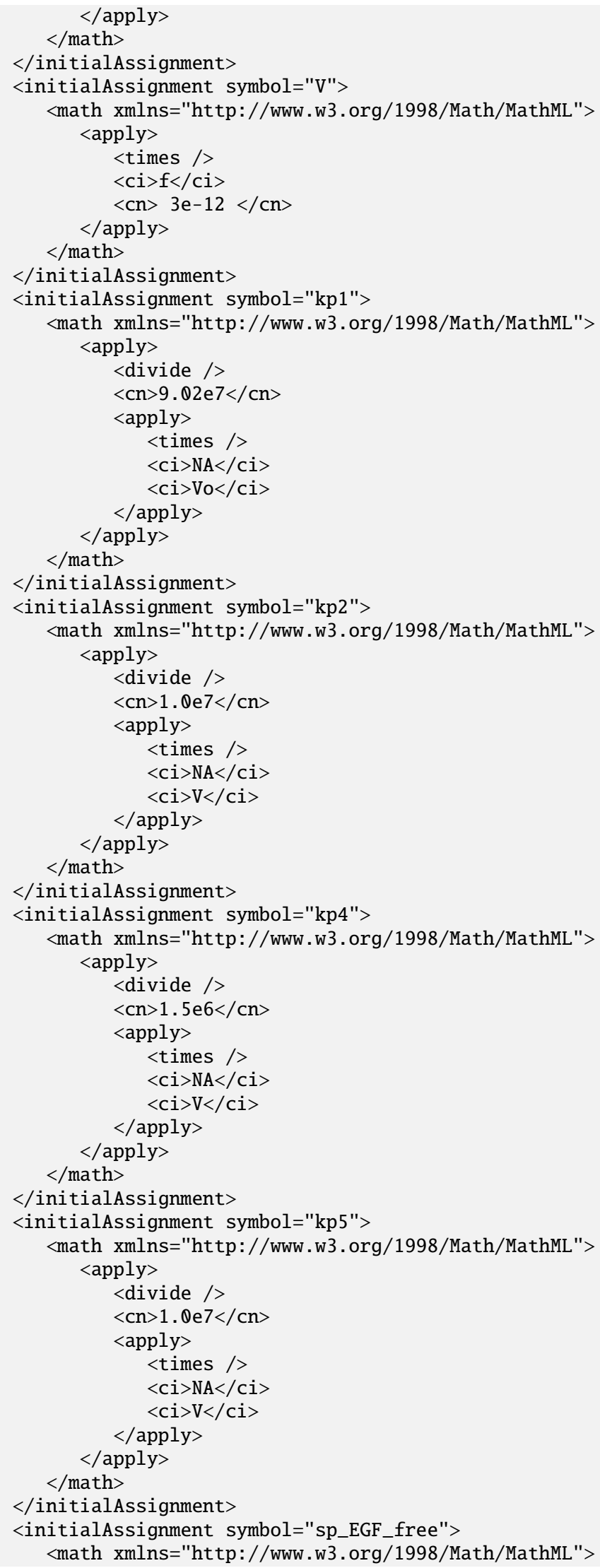




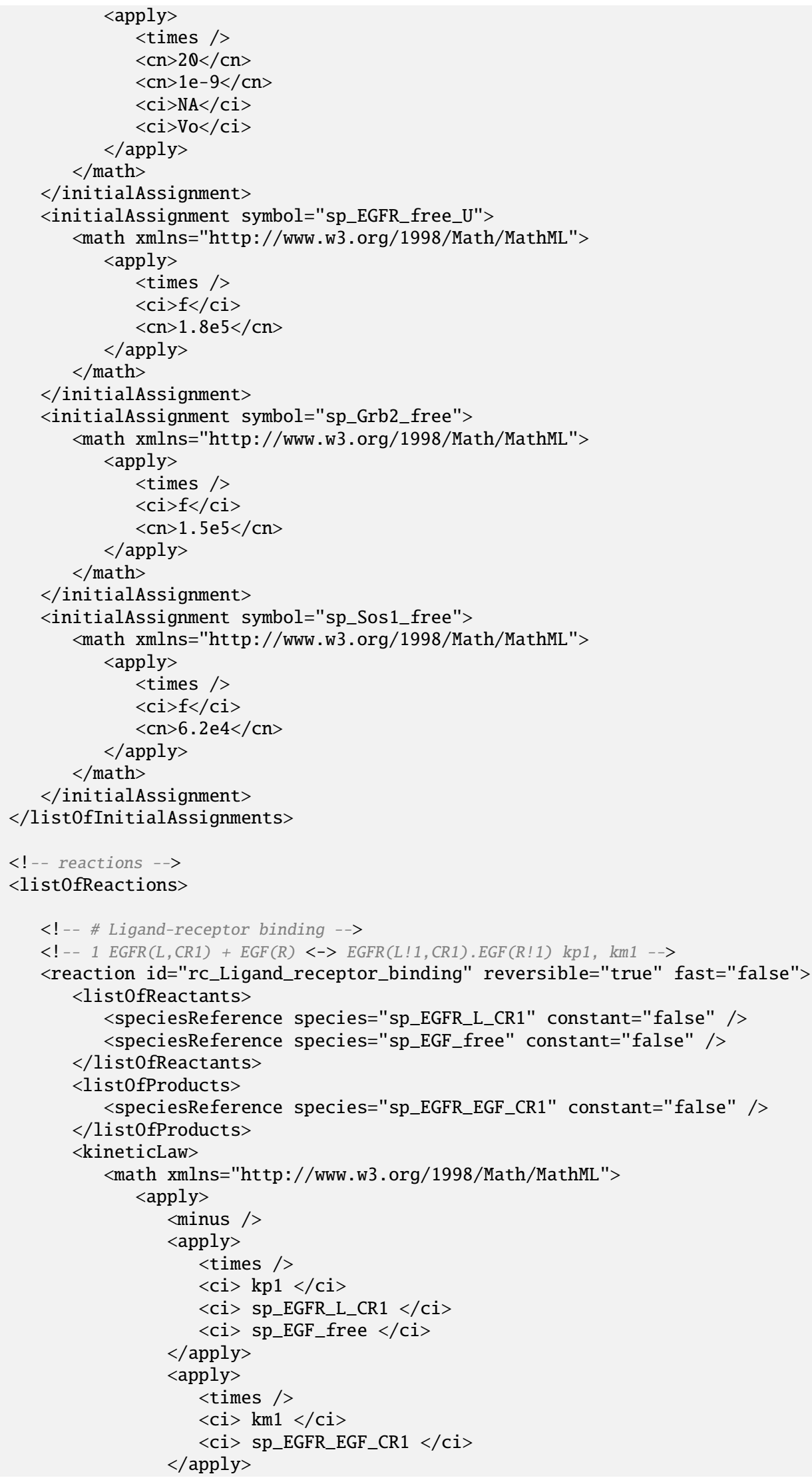




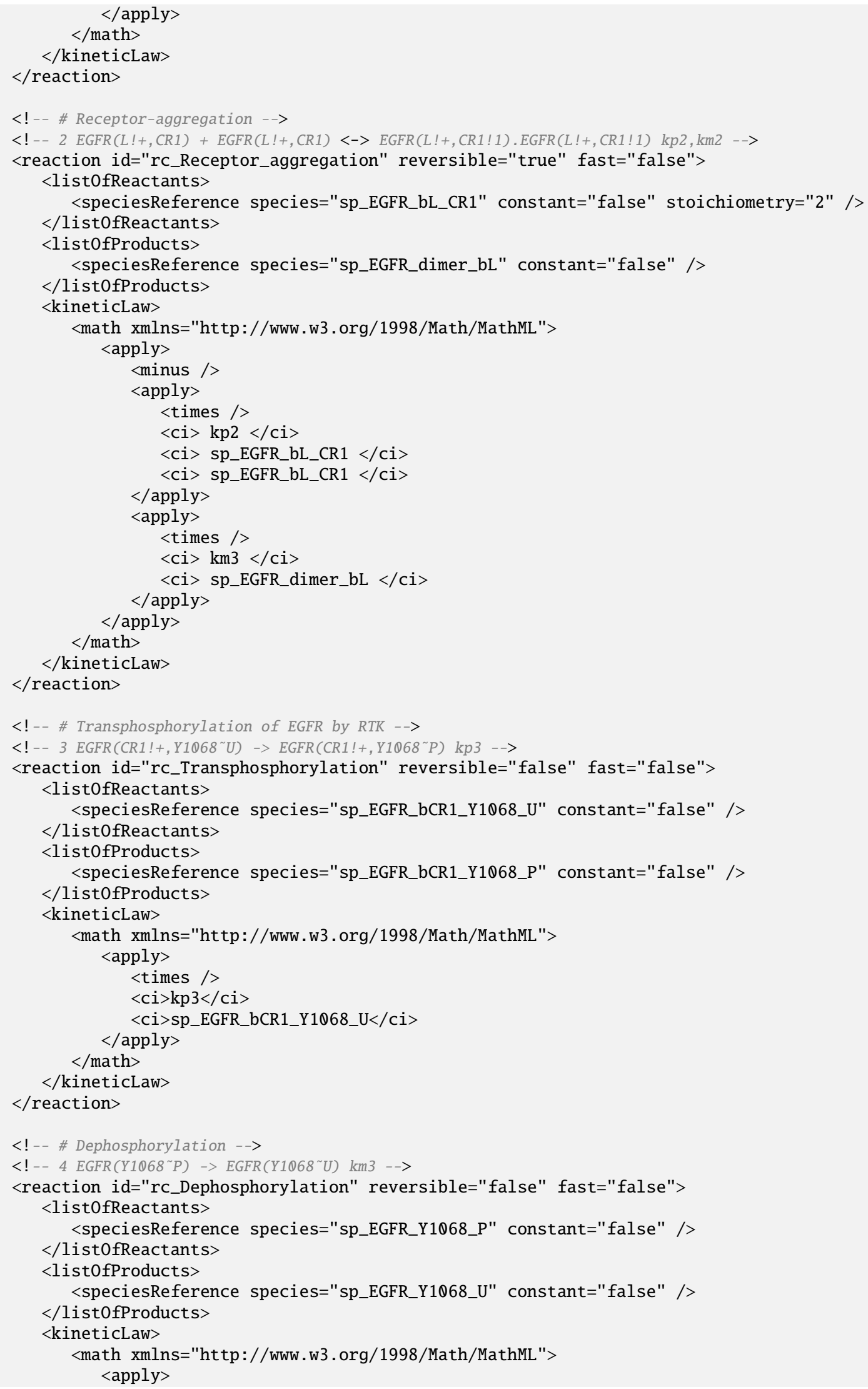




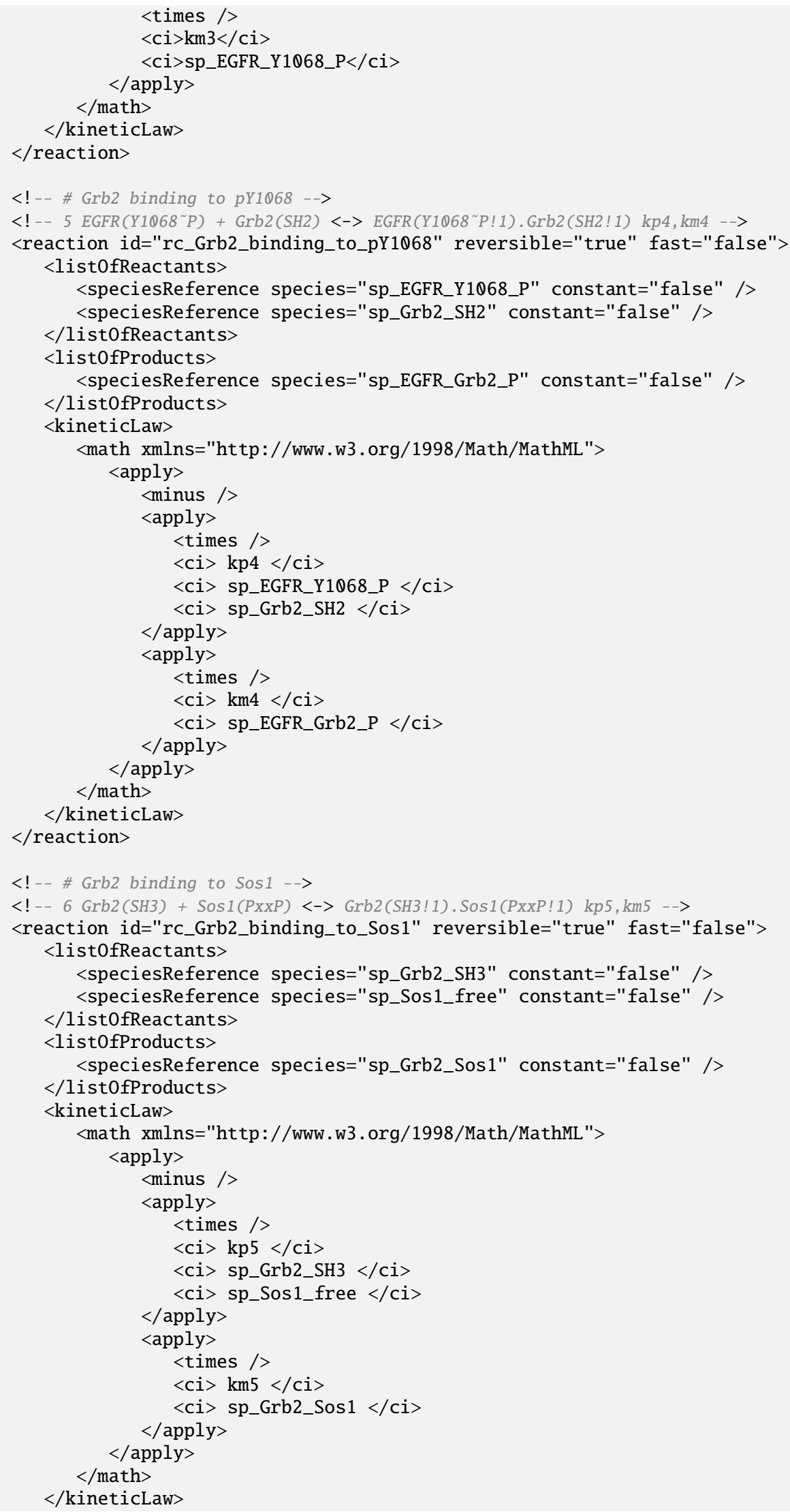




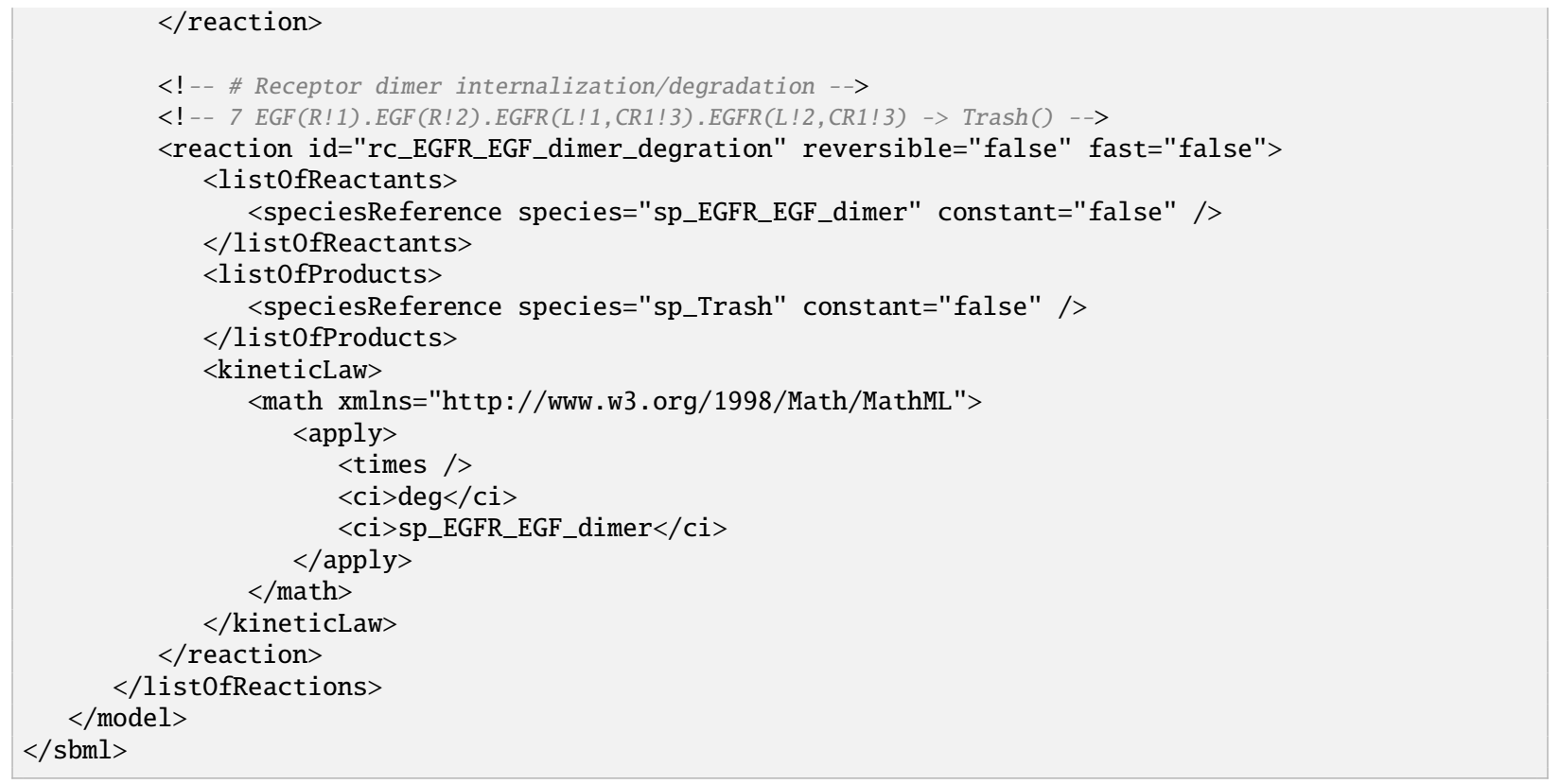

\subsection{Example from Kappa's documentation}

Here is the example "An Introduction to Kappa Syntax" at Kappa website (http://www.kappalanguage.org/ syntax.html).

Rule in English: "Unphosphorylated Site1 of A binds to Site1 of B"

Kappa Rule: A(Site1 u) ,B(Site1) -> A(Site1 u!1),B(Site1!1)

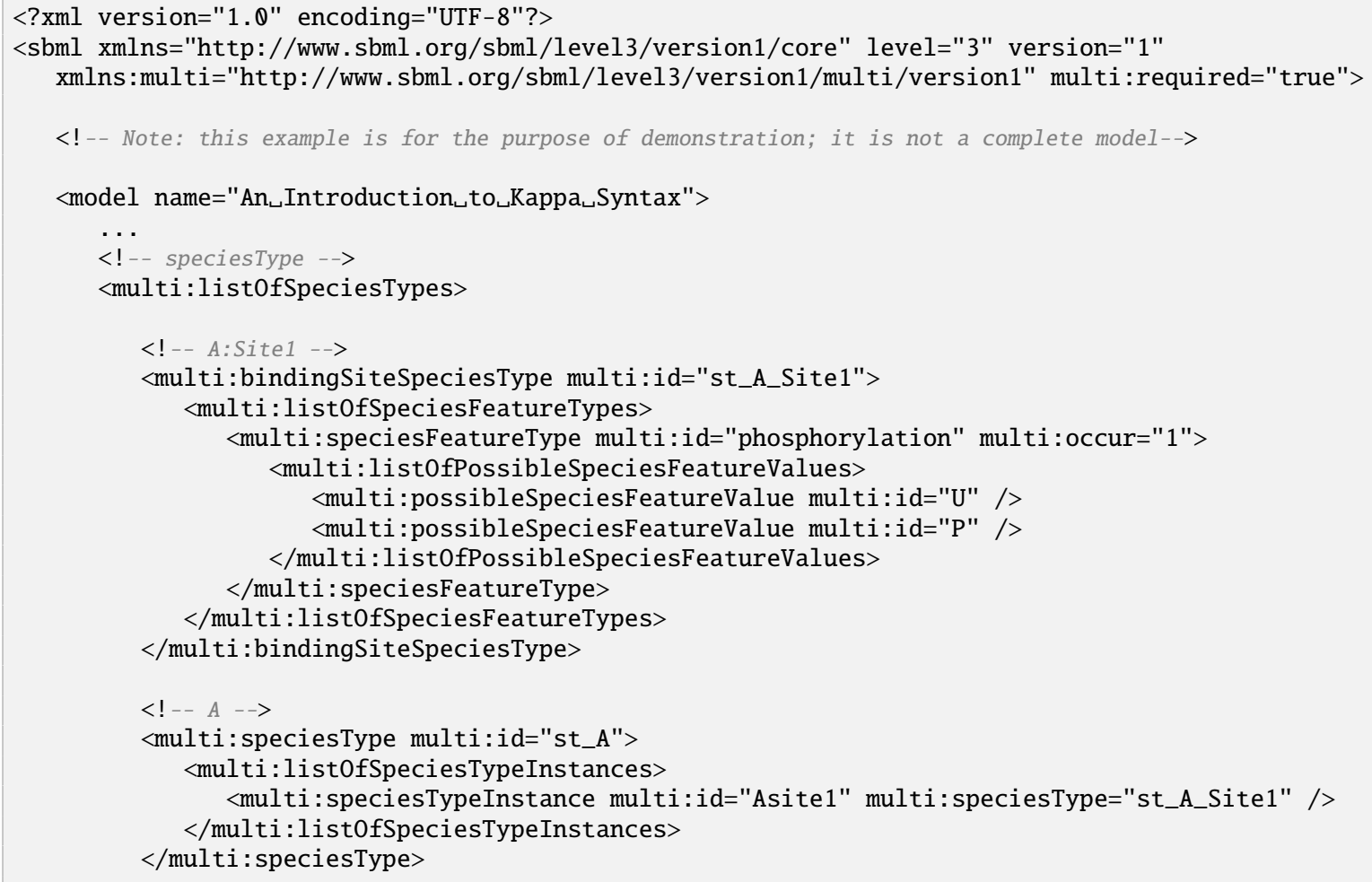


$<!--B:$ Site1 -->

$<$ multi:bindingSiteSpeciesType multi:id="st_B_Site1" />

$<!--B->$

$<$ multi:speciesType multi:id="st_B">

$<$ multi:list0fSpeciesTypeInstances>

$<$ multi:speciesTypeInstance multi:id="Bsite1" multi:speciesType="st_B_Site1" /> $</$ multi:list0fSpeciesTypeInstances $>$

$</$ multi:speciesType $>$

$<!--A \cdot B->$

$<$ multi:speciesType multi:id="st_AB">

$<$ multi:list0fSpeciesTypeInstances>

<multi:speciesTypeInstance multi:id="A" multi:speciesType="st_A" />

$<$ multi:speciesTypeInstance multi:id="B" multi:speciesType="st_B" />

$</$ multi:list0fSpeciesTypeInstances $>$

$<$ multi:list0fInSpeciesTypeBonds>

$<$ multi:inSpeciesTypeBond multi:bindingSite1="Asite1"

multi:bindingSite2="Bsite1" />

$<$ multi:list0fInSpeciesTypeBonds $>$

$</$ multi:speciesType $>$

$\ldots$

$</$ multi:list0fSpeciesTypes $>$

$<$ !-- species -->

$<$ list0fSpecies $>$

$<$ !-- species $A$ with free unphosphorylated Site1 -->

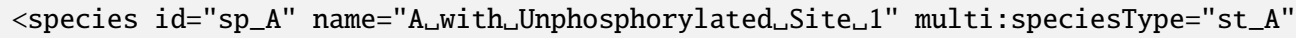

hasOnlySubstanceUnits="false" boundaryCondition="false" constant="false">

$<$ multi:list0fOutwardBindingSites>

$<$ multi: outwardBindingSite multi: component="Asite1"

multi:bindingStatus="unbound" />

$<$ /multi:list0fOutwardBindingSites $>$

$<$ multi:list0fSpeciesFeatures>

<multi:speciesFeature multi:speciesFeatureType="phosphorylation"> $<$ multi:list0fSpeciesFeatureValues >

$<$ multi:speciesFeatureValue multi:value="U" />

$</$ multi:list0fSpeciesFeatureValues $>$

$</$ multi:speciesFeature $>$

$</$ multi:list0fSpeciesFeatures $>$

$</$ species $>$

$<$ !-- species $B$ with free Site 1 -->

<species id="sp_B" name="B" multi: speciesType="st_B" hasOnlySubstanceUnits="false"

boundaryCondition="false" constant="false">

$<$ multi:list0fOutwardBindingSites>

$<$ multi: outwardBindingSite multi: component="sti_B_Site1"

multi:bindingStatus="unbound" />

$<$ /multi:list0fOutwardBindingSites $>$

$</$ species $>$

$<!--$ species $A B$ : unphosphorylated -->

<species id="sp_AB" name="AB" multi: speciesType="st_AB" hasOnlySubstanceUnits="false" boundaryCondition="false" constant="false">

$<$ multi:list0fSpeciesFeatures>

$<$ multi:speciesFeature multi:speciesFeatureType="phosphorylation"> $<$ multi:list0fSpeciesFeatureValues>

$<$ multi:speciesFeatureValue multi:value="U" />

$<$ /multi:list0fSpeciesFeatureValues $>$

$</$ multi:speciesFeature $>$

$</$ multi:list0fSpeciesFeatures $>$

$</$ species $>$

$\ldots$

$</$ list0fSpecies $>$

$<$ !-- reactions --> 


\section{$<$ list0fReactions $>$}

$<$ !-- Unphosphorylated Site1 of $A$ binds to Site1 of $B->$

$<$ ! -- Kappa Rule: A(Site1 u),B(Site1) $\rightarrow$ A(Site1 u!1),B(Site1!1) - >

$<$ reaction id="rc_AB" reversible="false" fast="false">

$<$ list0fReactants $>$

<speciesReference species="sp_A" constant="false" /> <speciesReference species="sp_B" constant="false" />

$</$ list0fReactants $>$

$<$ list0fProducts $>$

<speciesReference species="sp_AB" constant="false" />

$</$ list0fProducts $>$

$<$ kineticLaw $>$

$</$ kineticLaw $>$

$</$ reaction $>$

. .

$</$ list0fReactions $>$

$</$ model $>$

$</$ sbml $>$ 


\section{A Validation of SBML documents using Multi constructs}

This section summarizes all the conditions that should be true of an SBML Level 3 Version 1 model that uses the Multi package. We use the same conventions that are used in the SBML Level 3 Version 1 Core specification document. In particular, there are different degrees of rule strictness. Formally, the differences are expressed in the statement of a rule: either a rule states that a condition must be true, or a rule states that it should be true. Rules of the former kind are strict SBML validation rules-a model encoded in SBML must conform to all of them in order to be considered valid. Rules of the latter kind are consistency rules. To help highlight these differences, we use the three symbols next to the rule numbers as described in section A of the SBML Level 3 Version 1 Core specification document:

$\square$ A checked box indicates a requirement for SBML conformance. If a model does not follow this rule, it does not conform to the Multi package specification. (Mnemonic intention behind the choice of symbol: "This must be checked.")

A A triangle indicates a recommendation for model consistency. If a model does not follow this rule, it is not considered strictly invalid as far as the Multi package specification is concerned; however, it indicates that the model contains a physical or conceptual inconsistency. (Mnemonic intention behind the choice of symbol: "This is a cause for warning.")

$\star$ A star indicates a strong recommendation for good modeling practice. This rule is not strictly a matter of SBML encoding, but the recommendation comes from logical reasoning. As in the previous case, if a model does not follow this rule, it is not considered an invalid SBML encoding. (Mnemonic intention behind the choice of symbol: "You're a star if you heed this.")

The validation rules listed in the following subsections are all stated or implied in the rest of this specification document. They are enumerated here for convenience. Unless explicitly stated, all validation rules concern objects and attributes specifically defined in the Multi package.

For convenience and brevity, we use the shorthand "multi: $\mathrm{x}$ " to stand for an attribute or element name $\mathrm{x}$ in the namespace for the Multi package, using the namespace prefix multi. We use "multi : $x$ " because it is shorter than to write a full explanation everywhere we refer to an attribute or element in the Multi package namespace.

\section{General rules about the Multi package}

multi-10101 $\square \quad$ To conform to Version 1 of the Multi package specification for SBML Level 3, an SBML document must declare the use of the following XML Namespace:

"http://www. sbml .org/sbml/level3/version1/multi/version1". (References: SBML Level 3 Package Specification for Multi Version 1, Section 3.1 on page 9.)

multi-10102 $\square \quad$ Wherever they appear in an SBML document, elements and attributes from the Multi package must be declared either implicitly or explicitly to be in the XML namespace

"http://www . sbml .org/sbml/level3/version1/multi/version1". (References: SBML Level 3 Package Specification for Multi Version 1, Section 3.1 on page 9.)

\section{General rules about MathML content in the Multi package}

multi-10201 $\square \quad$ A ci element in a Math object may have the optional attributes multi : speciesReference and multi : representationType. No other attributes from the Multi namespace are permitted on a ci element. (References: Section 3.26 on page 33).

multi-10202 $\square \quad$ The value of the multi:speciesReference attribute on a given ci element must be the identifier of a SpeciesReference object within the same reaction. (References: Section 3.26.1 on page 33.) 
multi-10203 $\square \quad$ The value of the multi:representationType attribute on a given ci element must conform to the syntax of the Multi data type RepresentationType. (References: Section 3.26.2 on page 34.)

\section{General rules about identifiers}

multi-10301 $\varangle \quad$ (Extends validation rule \#10301 in the SBML Level 3 Version 1 Core specification.) Within a Model object, the values of the attributes id and multi :id on every instance of the following classes of objects must be unique across the set of all id and multi:id attribute values of all such objects in a model: the Model itself, plus all contained FunctionDefinition, Compartment, Species, Reaction, SpeciesReference, ModifierSpeciesReference, Event, and Parameter objects, plus the SpeciesType and PossibleSpeciesFeatureValue objects defined by the Multi package, and any objects defined by any other package with package: id attributes defined as falling in the 'SId' namespace. (References: Section 3.27 on page 35.)

multi-10302 $\checkmark \quad$ The value of a multi:id attribute must always conform to the syntax of the SBML data type SId. (References: SBML Level 3 Version 1 Core, Section 3.1.7.)

multi-10303 $\square \quad$ The value of a multi : name attribute must always conform to the syntax of type string. (References: SBML Level 3 Version 1 Core, Section 3.1.1.)

multi-10304 $\square$

The value of a multi : id attribute on SpeciesTypelnstance objects must be unique across the set of all multi:id attribute values of all the SpeciesTypelnstance objects under the direct parent SpeciesType object in which it is located. (References: Section 3.11.1 on page 16 and Section 3.27 on page 35.)

multi-10305 $\checkmark \quad$ The value of a multi:id attribute on SpeciesTypeComponentIndex objects must be unique across the set of all multi:id attribute values of all the SpeciesTypeComponentIndex objects under the direct parent SpeciesType object in which it is located. (References: Section 3.12.1 on page 17 and Section 3.27 on page 35.)

multi-10306 $\square$

The value of a multi : id attribute on InSpeciesTypeBond objects must be unique across the set of all multi:id attribute values of all the InSpeciesTypeBond objects under the direct parent SpeciesType object in which it is located. (References: Section 3.13.1 on page 19 and Section 3.27 on page 35.$)$

multi-10307 $\nabla$

multi-10308 $\square$

multi-10309 $\square$

multi-10310 $\square$

multi-10311 $\square$
The value of a multi:id attribute on SpeciesFeatureType objects must be unique across the set of all multi:id attribute values of all the SpeciesFeatureType objects under the direct parent SpeciesType object in which it is located. (References: Section 3.9.1 on page 14 and Section 3.27 on page 35.)

The value of a multi:id attribute on SubListOfSpeciesFeatures objects must be unique across the set of all id and multi:id attribute values of all objects in the Species object in which it is located. (References: Section 3.17.1 on page 25 and Section 3.27 on page 35.)

The value of a multi:id attribute on SpeciesFeature objects must be unique across the set of all id and multi : id attribute values of all objects in the Species object in which it is located. (References: Section 3.18.1 on page 26 and Section 3.27 on page 35.)

The value of a multi:id attribute on CompartmentReference objects must be unique across the set of all id and multi:id attribute values of all objects in the Compartment object in which it is located. (References: Section 3.6.1 on page 12 and Section 3.27 on page 35.)

The value of a multi: compartment attribute on SpeciesType objects must conform to the syntax of the SBML data type SIdRef. (References: Section 3.8.2 on page 13.) 
multi-10312 $\square$

multi-10313 $\square$

multi-10314 $\square$

multi-10315 $\square$

multi-10316 $\square$

multi-10317 $\square$

multi-10318 $\square$

multi-10319 $\square$

multi-10320 $\square$

multi-10321 $\square$

multi-10322 $\square$

multi-10323 $\nabla$

multi-10324 $\square$

multi-10325 $\square$

multi-10326 $\nabla$

multi-10327 $\square$

multi-10328 $\square$

multi-10329 $\nabla$
The value of a multi : numericValue attribute on PossibleSpeciesFeatureValue objects must conform to the syntax of the SBML data type SIdRef. (References: Section 3.10.2 on page 15.)

The value of a multi : speciesType attribute on SpeciesTypelnstance objects must conform to the syntax of the SBML data type SIdRef. (References: Section 3.11.2 on page 16.)

The value of a multi : compartmentReference attribute on SpeciesTypelnstance objects must conform to the syntax of the SBML data type SIdRef. (References: Section 3.11.3 on page 16.)

The value of a multi: component attribute on SpeciesTypeComponentIndex objects must conform to the syntax of the SBML data type SIdRef. (References: Section 3.12.2 on page 17.)

The value of a multi : identi fyingParent attribute on SpeciesTypeComponentlndex objects must conform to the syntax of the SBML data type SIdRef. (References: Section 3.12.3 on page 18.)

The value of a multi : bindingSite 1 attribute on InSpeciesTypeBond objects must conform to the syntax of the SBML data type SIdRef. (References: Section 3.13.2 on page 20.)

The value of a multi : bindingSite2 attribute on InSpeciesTypeBond objects must conform to the syntax of the SBML data type SIdRef. (References: Section 3.13.2 on page 20.)

The value of a multi : speciesType attribute on Species objects must conform to the syntax of the SBML data type SIdRef. (References: Section 3.15.1 on page 22.)

The value of a multi : component attribute on OutwardBindingSite objects must conform to the syntax of the SBML data type SIdRef. (References: Section 3.16.3 on page 23.)

The value of a multi : speciesFeatureType attribute on SpeciesFeature objects must conform to the syntax of the SBML data type SIdRef. (References: Section 3.18.2 on page 26.)

The value of a multi : component attribute on SpeciesFeature objects must conform to the syntax of the SBML data type SIdRef. (References: Section 3.18.4 on page 27.)

The value of a multi : value attribute on SpeciesFeatureValue objects must conform to the syntax of the SBML data type SIdRef. (References: Section 3.18.6 on page 27.)

The value of a multi : compartmentReference attribute on SimpleSpeciesReference objects must conform to the syntax of the SBML data type SIdRef. (References: Section 3.22 on page 30.)

The value of a multi : reactant attribute on SpeciesTypeComponentMapInProduct objects must conform to the syntax of the SBML data type SIdRef. (References: Section 3.24.2 on page 33.)

The value of a multi : reactantComponent attribute on SpeciesTypeComponentMaplnProduct objects must conform to the syntax of the SBML data type SIdRef. (References: Section 3.24.3 on page 33.)

The value of a multi : productComponent attribute on SpeciesTypeComponentMaplnProduct objects must conform to the syntax of the SBML data type SIdRef. (References: Section 3.24.4 on page 33.)

The value of a multi : compartmentType attribute on Compartment objects must conform to the syntax of the SBML data type SIdRef. (References: Section 3.5.2 on page 11.)

The value of a multi : compartment attribute on CompartmentReference objects must conform to the syntax of the SBML data type SIdRef. (References: Section 3.6.2 on page 12.) 


\section{Rules for extended SBML object}

multi-20101 $\square \quad$ The multi : required attribute is required on the $<$ sbml $>$ element in the Multi package. (References: SBML Level 3 Package Specification for Multi Version 1, Section 3.1 on page 9.)

multi-20102 $\square \quad$ The multi : required attribute on the <sbml> element must be Boolean. (References: SBML Level 3 Package Specification for Multi Version 1, Section 3.1 on page 9.)

multi-20103 $\square \quad$ The value of the multi:required attribute on the $<$ sbml $>$ element must be "true". (References: SBML Level 3 Package Specification for Multi Version 1, Section 3.1 on page 9.)

\section{Rules for extended Model objects}

multi-20201 $\square \quad$ There may be at most one ListOfSpeciesTypes container object within a Model object. (References: Section 3.3 on page 10.)

multi-20202 $\square \quad$ A ListOfSpeciesTypes object within an extended Model object is optional, but if present, must not be empty. (References: Section 3.3 on page 10.)

multi-20203 $\square \quad$ A ListOfSpeciesTypes object may have the optional SBML core attributes metaid and sboTerm. No other attributes from the SBML Level 3 Core namespace or the Multi namespace are permitted on a ListOfSpeciesTypes object. (References: Section 3.4.1 on page 11.)

multi-20204 $\square \quad$ Apart from the general notes and annotation subobjects permitted on all SBML objects, a ListOfSpeciesTypes container object may only contain SpeciesType objects. (References: Section 3.4.1 on page 11.)

\section{Rules for extended Compartment objects}

multi-20301 $\square \quad$ An extended Compartment object must have the required attribute multi:isType, and may also have the optional attribute multi : comparetmentType. No other attributes from the Multi namespace are permitted on an extended Compartment object. (References: Section 3.5 on page 11.)

multi-20302 $\square$

multi-20303 $\square$

multi-20304 $\square$

multi-20305 $\square$

multi-20306 $\nabla$

multi-20307 $\nabla$

multi-20308 $\nabla$
The value of a multi:isType attribute on an extended Compartment object must always confirm to the syntax of the SBML data type boolean. (References: Section 3.5.1 on page 11.)

The multi:isType attribute on an extended Compartment object is required. (References: Section 3.5.1 on page 11.)

The value of the multi: isType attribute of the Compartment object referenced by a CompartmentReference object must be the same as that of the multi:isType attribute of the parent Compartment object of the ListOfCompartmentReferences object which contains the CompartmentReference object. (References: Section 3.7 on page 12.)

The multi : compartmentType attribute on a Compartment object must not be defined if the value of the multi: isType is "true". (References: Section 3.5.2 on page 11.)

There may be at most one ListOfCompartmentReferences container object within a Compartment object. (References: Section 3.5.3 on page 12.)

A ListOfCompartmentReferences object within a Compartment object is optional, but if present, must not be empty. (References: Section 3.5.3 on page 12.)

A ListOfCompartmentReferences object may have the optional SBML core attributes metaid and sboTerm. No other attributes from the SBML Level 3 Core namespace or the Multi namespace are permitted on a ListOfCompartmentReferences object. (References: Section 3.5.3 on page 12.) 
multi-20309 $\square \quad$ Apart from the general notes and annotation subobjects permitted on all SBML objects, a ListOfCompartmentReferences container object may only contain CompartmentReference objects. (References: Section 3.5.3 on page 12.)

\section{Rules for SpeciesType objects}

multi-20401 $\square \quad$ A SpeciesType object may have the optional SBML Level 3 Core attributes metaid and sboTerm. No other attributes from the SBML Level 3 Core namespace are permitted on a SpeciesType object. (References: Section 3.8 on page 12).

multi-20402 $\square \quad$ A SpeciesType object may have the optional SBML Level 3 Core subobjects for notes and annotation. No other elements from the SBML Level 3 Core namespace are permitted on a SpeciesType object. (References: Section 3.8 on page 12).

multi-20403 $\square \quad$ A SpeciesType object must have the required attribute multi:id, and may have the optional attributes multi : name and multi : compartment. No other attributes from the Multi namespace are permitted on a SpeciesType object. (References: Section 3.8 on page 12.)

multi-20404 $\square \quad$ The value of the multi : compartment attribute, if set on a given SpeciesType object, must be the value of an id attribute on an existing Compartment object in the SId namespace of the parent Model object. (References: Section 3.8.2 on page 13.)

multi-20405 $\square \quad$ The various ListOf__ subobjects within a SpeciesType object are optional, but if present, these container objects must not be empty. Specifically, if any of the following classes of objects are present with a SpeciesType object, it must not be empty: ListOfSpeciesFeatureTypes, ListOfSpeciesTypelnstances, ListOfSpeciesTypeComponentIndexes and ListOfInSpeciesTypeBonds. (References: Section 3.8 on page 12.)

multi-20406 $\square \quad$ There may be at most one ListOfSpeciesFeatureTypes container object within a SpeciesType object. (References: Section 3.8 on page 12.)

multi-20407 $\square$

Apart from the general notes and annotation subobjects permitted on all SBML objects, a ListOfSpeciesFeatureTypes container object may only contain SpeciesFeatureType objects. (References: Section 3.8.3 on page 13.)

multi-20408 $\nabla$

A ListOfSpeciesFeatureTypes object may have the optional SBML core attributes metaid and sboTerm. No other attributes from the SBML Level 3 Core namespace or the Multi namespace are permitted on a ListOfSpeciesFeatureTypes object. (References: Section 3.8.3 on page 13.)

multi-20409 $\square \quad$ There may be at most one ListOfSpeciesTypelnstances container object within a SpeciesType object. (References: Section 3.8 on page 12.)

multi-20410 $\square$

Apart from the general notes and annotation subobjects permitted on all SBML objects, a ListOfSpeciesTypelnstances container object may only contain SpeciesTypelnstance objects. (References: Section 3.8.4 on page 13.)

multi-20411 $\square \quad$ A ListOfSpeciesTypelnstances object may have the optional SBML core attributes metaid and sboTerm. No other attributes from the SBML Level 3 Core namespace or the Multi namespace are permitted on a ListOfSpeciesTypelnstances. (References: Section 3.8.4 on page 13.)

multi-20412 $\square \quad$ There may be at most one ListOfSpeciesTypeComponentIndexes container object within a SpeciesType object. (References: Section 3.8 on page 12.)

multi-20413 $\square \quad$ Apart from the general notes and annotation subobjects permitted on all SBML objects, a ListOfSpeciesTypeComponentIndexes container object may only contain SpeciesTypeComponentIndex objects. (References: Section 3.8.6 on page 14.) 
multi-20414 $\square \quad$ A ListOfSpeciesTypeComponentIndexes object may have the optional SBML core attributes metaid and sboTerm. No other attributes from the SBML Level 3 Core namespace or the Multi namespace are permitted on a ListOfSpeciesTypeComponentIndexes object. (References: Section 3.8.6 on page 14.)

multi-20415 $\square \quad$ There may be at most one ListOfInSpeciesTypeBonds container object within a SpeciesType object. (References: Section 3.8 on page 12.)

multi-20416 $\square \quad$ Apart from the general notes and annotation subobjects permitted on all SBML objects, a ListOfInSpeciesTypeBonds container object may only contain InSpeciesTypeBond objects. (References: Section 3.8.5 on page 14.)

multi-20417 $\square \quad$ A ListOfInSpeciesTypeBonds object may have the optional SBML core attributes metaid and sboTerm. No other attributes from the SBML Level 3 Core namespace or the Multi namespace are permitted on a ListOfInSpeciesTypeBonds object. (References: Section 3.8.5 on page 14.)

\section{Rules for BindingSiteSpeciesType objects}

multi-20501 $\square \quad$ A BindingSiteSpeciesType object is not permitted to have any ListOfSpeciesTypelnstances subobject. (References: Section 3.8.7 on page 14).

\section{Rules for SpeciesFeatureType objects}

multi-20601 $\square \quad$ A SpeciesFeatureType object may have the optional SBML Level 3 Core attributes metaid and sboTerm. No other attributes from the SBML Level 3 Core namespace are permitted on a SpeciesFeatureType object. (References: Section 3.9 on page 14).

multi-20602 $\square \quad$ A SpeciesFeatureType object may have the optional SBML Level 3 Core subobjects for notes and annotation. No other elements from the SBML Level 3 Core namespace are permitted on a SpeciesFeatureType object. (References: Section 3.9 on page 14).

multi-20603 $\square \quad$ A SpeciesFeatureType object must have the required attributes multi:id and multi:occur, and may have the optional attribute multi : name. No other attributes from the Multi namespace are permitted on a SpeciesFeatureType object. (References: Section 3.9 on page 14.)

multi-20604 $\square \quad$ The value of the multi : occur attribute on a given SpeciesFeatureType object must conform to the syntax of the SBML data type positiveInteger. (References: Section 3.9.2 on page 14.)

multi-20605 $\square \quad$ One ListOfPossibleSpeciesFeatureValues subobject in a SpeciesFeatureType object is required. (References: Section 3.9.3 on page 14.)

multi-20606 $\square \quad$ A ListOfPossibleSpeciesFeatureValues object may have the optional SBML core attributes metaid and sboTerm. No other attributes from the SBML Level 3 Core namespace or the Multi namespace are permitted on a ListOfPossibleSpeciesFeatureValues object. (References: Section 3.9.3 on page 14.)

multi-20607 $\square \quad$ Apart from the general notes and annotation subobjects permitted on all SBML objects, a ListOfPossibleSpeciesFeatureValues container object may only contain PossibleSpeciesFeatureValue objects. (References: Section 3.9.3 on page 14.)

multi-20608 $\square \quad$ A ListOfPossibleSpeciesFeatureValues object must not be empty. (References: Section 3.9.3 on page 14.)

\section{Rules for PossibleSpeciesFeatureValue objects}

multi-20701 $\square \quad$ A PossibleSpeciesFeatureValue object may have the optional SBML Level 3 Core attributes metaid and sboTerm. No other attributes from the SBML Level 3 Core namespace are permit- 
ted on a PossibleSpeciesFeatureValue object. (References: Section 3.10 on page 15).

multi-20702 $\checkmark \quad$ A PossibleSpeciesFeatureValue object may have the optional SBML Level 3 Core subobjects for notes and annotation. No other elements from the SBML Level 3 Core namespace are permitted on a PossibleSpeciesFeatureValue object. (References: Section 3.10 on page 15).

multi-20703 $\varangle \quad$ A PossibleSpeciesFeatureValue object must have the required attribute multi:id, and may have the optional attributes multi : name and multi: numericValue. No other attributes from the Multi namespace are permitted on a PossibleSpeciesFeatureValue object. (References: Section 3.10 on page 15.)

multi-20704 $\varangle \quad$ The value of the multi : numericValue attribute on a given PossibleSpeciesFeatureValue object must be the identifier of a Parameter object defined in the same Model object. (References: Section 3.10.2 on page 15.)

\section{Rules for SpeciesTypelnstance objects}

multi-20801 $\checkmark \quad$ A SpeciesTypelnstance object may have the optional SBML Level 3 Core attributes metaid and sboTerm. No other attributes from the SBML Level 3 Core namespace are permitted on a SpeciesTypelnstance object. (References: Section 3.11 on page 16).

multi-20802 $\square \quad$ A SpeciesTypelnstance object may have the optional SBML Level 3 Core subobjects for notes and annotation. No other elements from the SBML Level 3 Core namespace are permitted on a SpeciesTypelnstance object. (References: Section 3.11 on page 16).

multi-20803 $\square \quad$ A SpeciesTypelnstance object must have the required attributes multi:id and multi:speciesType, and may have the optional attributes multi : name and multi : compartmentReference. No other attributes from the Multi namespace are permitted on a SpeciesTypelnstance object. (References: Section 3.11 on page 16.)

multi-20805 $\checkmark \quad$ The value of the multi:speciesType attribute on a given SpeciesTypelnstance object must be the identifier of a SpeciesType object defined in the same Model object. (References: Section 3.11 .2 on page 16.)

multi-20806 $\checkmark \quad$ The value of the multi : compartmentReference attribute, if present on a given SpeciesTypeInstance object, must be the identifier of a CompartmentReference object defined in the same Model object. (References: Section 3.11.3 on page 16.)

\section{Rules for SpeciesTypeComponentIndex objects}

multi-20901 $\checkmark \quad$ A SpeciesTypeComponentIndex object may have the optional SBML Level 3 Core attributes metaid and sboTerm. No other attributes from the SBML Level 3 Core namespace are permitted on a SpeciesTypeComponentIndex object. (References: Section 3.12 on page 17).

multi-20902 $\square \quad$ A SpeciesTypeComponentIndex object may have the optional SBML Level 3 Core subobjects for notes and annotation. No other elements from the SBML Level 3 Core namespace are permitted on a SpeciesTypeComponentIndex object. (References: Section 3.12 on page 17).

multi-20903 $\square \quad$ A SpeciesTypeComponentIndex object must have the required attributes multi:id and multi : compartment, and may have the optional attributes multi : name and multi :identifyingParent. No other attributes from the Multi namespace are permitted on a SpeciesTypeComponentIndex object. (References: Section 3.12 on page 17.)

multi-20904 $\varangle \quad$ The value of the multi : component attribute on a given SpeciesTypeComponentIndex object must be the identifier of a SpeciesTypelnstance object, or a SpeciesTypeComponentIndex object under the SpeciesType object that this SpeciesTypeComponentIndex object belongs to, or the SpeciesType object itself. (References: Section 3.12.2 on page 17.) 
multi-20907 $\square \quad$ The value of the multi:identifyingParent attribute on a given SpeciesTypeComponentIndex object must be the identifier of a component object under the SpeciesType object that this SpeciesTypeComponentIndex object belongs to. A component object can be an object of SpeciesTypelnstance, SpeciesTypeComponentIndex or SpeciesType. (References: Section 3.12 .3 on page 18.)

\section{Rules for InSpeciesTypeBond objects}

multi-21101 $\checkmark \quad$ An InSpeciesTypeBond object may have the optional SBML Level 3 Core attributes metaid and sboTerm. No other attributes from the SBML Level 3 Core namespace are permitted on an InSpeciesTypeBond object. (References: Section 3.13 on page 19).

multi-21102 $\square \quad$ An InSpeciesTypeBond object may have the optional SBML Level 3 Core subobjects for notes and annotation. No other elements from the SBML Level 3 Core namespace are permitted on an InSpeciesTypeBond object. (References: Section 3.13 on page 19).

multi-21103 $\square \quad$ An InSpeciesTypeBond object must have the required attributes, multi:bindingSite1 and multi : bindingSite2, and may have the optional attributes, multi:id and multi :name. No other attributes from the Multi namespace are permitted on an InSpeciesTypeBond object. (References: Section 3.13 on page 19.)

multi-21104 $\square \quad$ The value of the multi : bindingSite1 attribute on a given InSpeciesTypeBond object must be the identifier of a SpeciesTypelnstance object or SpeciesTypeComponentIndex which ultimately reference a object of BindingSiteSpeciesType. (References: Section 3.13.2 on page 20.)

multi-21105 $\square \quad$ The value of the multi : bindingSite2 attribute on a given InSpeciesTypeBond object must be the identifier of a SpeciesTypelnstance object or SpeciesTypeComponentIndex which ultimately reference a object of BindingSiteSpeciesType. (References: Section 3.13.2 on page 20.)

multi-21106 $\square \quad$ The multi : bindingSite1 and multi : bindingSite2 attributes must not reference the same BindingSiteSpeciesType object. (References: Section 3.13.2 on page 20.)

\section{Rules for extended Species objects}

multi-21201 $\square \quad$ A Species object may have the optional attribute, multi:speciesType. No other attributes from the Multi namespace are permitted on a Species object. (References: Section 3.14 on page 22.)

multi-21202 $\checkmark \quad$ The value of a multi:speciesType attribute, if present on a Species object, must be the identifier of a SpeciesType object. (References: Section 3.15.1 on page 22.)

multi-21203 $\square \quad$ Two ListOf__ subobjects with a Species object are optional, but if present, these container object must not be empty. Specifically, if any of the following two classes of objects are present on the Species object, it must not be empty: ListOfOutwardBindingSites and ListOfSpeciesFeatures. (References: Section 3.14 on page 22.)

multi-21204 $\nabla \quad$ A ListOfOutwardBindingSites object may have the optional SBML core attributes metaid and sboTerm. No other attributes from the SBML Level 3 Core namespace or the Multi namespace are permitted on a ListOfOutwardBindingSites object. (References: Section 3.15.2 on page 22.)

multi-21205 $\nabla \quad$ Apart from the general notes and annotation subobjects permitted on all SBML objects, a ListOfOutwardBindingSites container object may only contain OutwardBindingSite objects. (References: Section 3.15.2 on page 22.)

multi-21206 $\square \quad$ A ListOfSpeciesFeatures object may have the optional SBML core attributes metaid and sboTerm. No other attributes from the SBML Level 3 Core namespace or the Multi namespace are permitted on a ListOfSpeciesFeatures object. (References: Section 3.15.3 on page 23.) 
multi-21207 $\square$

multi-21208 $\square$

multi-21209 $\square$

multi-21210 $\square$

multi-21211 $\square$

multi-21212 $\square$

multi-21213 $\square$

multi-21214 $\square$

multi-21215 $\square$

multi-21216 $\square$
A SubListOfSpeciesFeatures object may have the optional attributes multi : id, multi: name, multi: component, and the required attribute multi:relation. No other attributes from the Multi namespace are permitted on a SubListOfSpeciesFeatures object. (References: Section 3.17 on page 25.)

The value of the multi:relation attribute, if presented on a SubListOfSpeciesFeatures object, must conform to the syntax of the Multi data type Relation. (References: Section 3.17.2 on page 25.)

Apart from the general notes and annotation subobjects permitted on all SBML objects, a ListOfSpeciesFeatures container object may only contain SpeciesFeature and/or SubListOfSpeciesFeatures objects. (References: Section 3.15.3 on page 23.)

A SubListOfSpeciesFeatures object may have the optional SBML core attributes metaid and sboTerm. No other attributes from the SBML Level 3 Core namespace are permitted on a SubListOfSpeciesFeatures object. (References: Section 3.17 on page 25.)

Apart from the general notes and annotation subobjects permitted on all SBML objects, a SubListOfSpeciesFeatures container object may only contain SpeciesFeature objects. (References: Section 3.17 on page 25.)

The value of the multi:component attribute on a given SubListOfSpeciesFeatures object must be the identifier of an object of SpeciesTypelnstance, SpeciesTypeComponentIndex or SpeciesType which contains the SpeciesFeature objects in this subList0fSpeciesFeatures. (References: Section 3.17.3 on page 25.)

A species must have its speciesType attribute defined when it has a list0f0utwardBindingSites. (References: Section 3.14 on page 22.)

A species must have its speciesType attribute defined when it has a list0fSpeciesFeatures. (References: Section 3.14 on page 22.)

The relation attribute of a subListOfSpeciesFeatures can only have the value "and" if any speciesFeature involved references a speciesFeatureType with "occur > 1". (References: Section 3.17.2 on page 25.)

A SubListOfSpeciesFeatures object must have at least two speciesFeatures. (References: Section 3.17 on page 25.)

\section{Rules for OutwardBindingSite objects}

multi-21301 $\square \quad$ An OutwardBindingSite object may have the optional SBML Level 3 Core attributes metaid and sboTerm. No other attributes from the SBML Level 3 Core namespace are permitted on an OutwardBindingSite object. (References: Section 3.16 on page 23).

multi-21302 $\checkmark \quad$ An OutwardBindingSite object may have the optional SBML Level 3 Core subobjects for notes and annotation. No other elements from the SBML Level 3 Core namespace are permitted on an OutwardBindingSite object. (References: Section 3.16 on page 23).

multi-21303 $\checkmark \quad$ An OutwardBindingSite object must have the required attributes, multi:bindingStatus and multi:component, and may have the optional attributes multi:id and multi:name. No other attributes from the Multi namespace are permitted on an OutwardBindingSite object. (References: Section 3.16 on page 23.) 
multi-21304 $\square$

multi-21305 $\square$

multi-21306 $\square$
The value of the multi : bindingStatus attribute on a given OutwardBindingSite object must conform to the syntax of the Multi data type BindingStatus. (References: Section 3.16.2 on page 23.)

The value of the multi : component attribute on a given OutwardBindingSite object must be the identifier of an object of SpeciesTypelnstance, SpeciesTypeComponentIndex or SpeciesType which ultimately reference an object of BindingSiteSpeciesType. (References: Section 3.16.3 on page 23.)

An outwardBindingSi te cannot be a binding site referenced by any inSpeciesTypeBond in the species. (References: Section 3.16.3 on page 23.)

\section{Rules for SpeciesFeature objects}

multi-21401 $\checkmark \quad$ A SpeciesFeature object may have the optional SBML Level 3 Core attributes metaid and sboTerm. No other attributes from the SBML Level 3 Core namespace are permitted on a SpeciesFeature object. (References: Section 3.18 on page 25).

multi-21402 $\varangle \quad$ A SpeciesFeature object may have the optional SBML Level 3 Core subobjects for notes and annotation. No other elements from the SBML Level 3 Core namespace are permitted on a SpeciesFeature object. (References: Section 3.18 on page 25).

multi-21403 $\nabla$

A SpeciesFeature object must have the required attributes, multi:speciesFeatureType and multi:occur, and may have the optional attributes, multi:id, multi:name, and multi:component. No other attributes from the Multi namespace are permitted on a SpeciesFeature object. (References: Section 3.18 on page 25.)

multi-21404 $\square$

The value of the multi:speciesFeatureType attribute on a given SpeciesFeature object must be the identifier of a SpeciesFeatureType object which is in the SpeciesType object referenced by the Species object containing this SpeciesFeature object. (References: Section 3.18.2 on page 26.)

multi-21405 $\square$

The value of the multi : occur attribute on a given SpeciesFeature object must conform to the syntax of the SBML data type positiveInteger. The value of the multi : occur attribute must not be larger than that of the multi : occur attribute of the SpeciesFeatureType object referenced by this SpeciesFeature object. (References: Section 3.18.3 on page 26.)

multi-21406 $\nabla$

The value of the multi : component attribute on a given SpeciesFeature object must be the identifier of an object of SpeciesTypelnstance, SpeciesTypeComponentIndex or SpeciesType which contains this SpeciesFeature object. (References: Section 3.18.4 on page 27.)

multi-21407 $\square \quad$ One and only one ListOfSpeciesFeatureValues subobject within a SpeciesFeature object is required. (References: Section 3.18.5 on page 27.)

multi-21408 $\square \quad$ A ListOfSpeciesFeatureValues object must not be empty. (References: Section 3.18 .5 on page 27.)

multi-21409 $\checkmark \quad$ A ListOfSpeciesFeatureValues object may have the optional SBML core attributes metaid and sboTerm. No other attributes from the SBML Level 3 Core namespace or the Multi namespace are permitted on a ListOfSpeciesFeatureValues object. (References: Section 3.18.5 on page 27.)

multi-21410 $\square \quad$ Apart from the general notes and annotation subobjects permitted on all SBML objects, a ListOfSpeciesFeatureValues container object may only contain SpeciesFeatureValue objects. (References: Section 3.18.5 on page 27.) 


\section{Rules for SpeciesFeatureValue objects}

multi-21501 $\checkmark \quad$ A SpeciesFeatureValue object may have the optional SBML Level 3 Core attributes metaid and sboTerm. No other attributes from the SBML Level 3 Core namespace are permitted on a SpeciesFeatureValue object. (References: Section 3.18.6 on page 27).

multi-21502 $\checkmark \quad$ A SpeciesFeatureValue object may have the optional SBML Level 3 Core subobjects for notes and annotation. No other elements from the SBML Level 3 Core namespace are permitted on a SpeciesFeatureValue object. (References: Section 3.18.6 on page 27).

multi-21503 $\checkmark \quad$ A SpeciesFeatureValue object must have the required attribute multi:value. No other attributes from the Multi namespace are permitted on a SpeciesFeatureValue object. (References: Section 3.18.6 on page 27.)

multi-21504 $\nabla \quad$ The value of the multi : value attribute on a given SpeciesFeatureValue object must be the identifier of a PossibleSpeciesFeatureValue object defined in the SpeciesFeatureType object referenced by the SpeciesFeature object containing this SpeciesFeatureValue object. (References: Section 3.18.6 on page 27.)

\section{Rules for IntraSpeciesReaction objects}

multi-21601 $\square \quad$ An IntraSpeciesReaction object may have the optional SBML Level 3 Core attributes metaid and sboTerm. No other attributes from the SBML Level 3 Core namespace and the Multi namespace are permitted on an IntraSpeciesReaction object. (References: Section 3.21 on page 30).

multi-21602 $\varangle \quad$ An IntraSpeciesReaction object may have the optional SBML Level 3 Core subobjects for notes and annotation. No other elements from the SBML Level 3 Core namespace are permitted on an IntraSpeciesReaction object. (References: Section 3.21 on page 30 ).

\section{Rules for extended SimpleSpeciesReference objects}

multi-21701 $\square \quad$ An extended SimpleSpeciesReference object may have the optional attribute, multi : compartmentReference. No other attributes from the Multi namespace are permitted on a SimpleSpeciesReference object. (References: Section 3.22 on page 30.)

multi-21702 $\square \quad$ The value of a multi : compartmentReference attribute, if present on a SimpleSpeciesReference object, must be the identifier of a CompartmentReference object. (References: Section 3.22 on page 30.)

\section{Rules for extended SpeciesReference objects}

multi-21801 $\checkmark \quad$ A ListOfSpeciesTypeComponentMapsInProduct object within an extended SpeciesReference object is optional, but if present, must not be empty. (References: Section 3.23.1 on page 32.)

multi-21802 $\checkmark \quad$ A ListOfSpeciesTypeComponentMapsInProduct object may have the optional SBML core attributes metaid and sboTerm. No other attributes from the SBML Level 3 Core namespace or the Multi namespace are permitted on a ListOfSpeciesTypeComponentMapsInProduct object. (References: Section 3.23.1 on page 32.)

multi-21803 $\nabla \quad$ Apart from the general notes and annotation subobjects permitted on all SBML objects, a ListOfSpeciesTypeComponentMapsInProduct container object may only contain SpeciesTypeComponentMapInProduct objects. (References: Section 3.23.1 on page 32.) 


\section{Rules for SpeciesTypeComponentMapInProduct objects}

multi-21901 $\square \quad$ A SpeciesTypeComponentMapInProduct object may have the optional SBML Level 3 Core attributes metaid and sboTerm. No other attributes from the SBML Level 3 Core namespace are permitted on a SpeciesTypeComponentMapInProduct object. (References: Section 3.24 on page 32).

multi-21902 $\varangle \quad$ A SpeciesTypeComponentMapInProduct object may have the optional SBML Level 3 Core subobjects for notes and annotation. No other elements from the SBML Level 3 Core namespace are permitted on a SpeciesTypeComponentMapInProduct object. (References: Section 3.24 on page 32).

multi-21903 $\nabla \quad$ A SpeciesTypeComponentMapInProduct object must have the required attributes multi :reactant, multi:reactantComponent, and multi:productComponent, and may have the optional attributes multi:id and multi : name. No other attributes from the Multi namespace are permitted on a SpeciesTypeComponentMapInProduct object. (References: Section 3.24 on page 32.)

multi-21904 $\square \quad$ The value of the multi:reactant attribute on a given SpeciesTypeComponentMaplnProduct object must be the identifier of a reactant SpeciesReference object within a reaction. (References: Section 3.24.2 on page 33.)

multi-21905 $\checkmark \quad$ The value of the multi:reactantComponent attribute on a given SpeciesTypeComponentMapInProduct object must be the identifier of an object of SpeciesTypelnstance, SpeciesTypeComponentIndex or SpeciesType. (References: Section 3.24.3 on page 33.)

multi-21906 $\nabla \quad$ The value of the multi : productComponent attribute on a given SpeciesTypeComponentMapInProduct object must be the identifier of an object of SpeciesTypelnstance, SpeciesTypeComponentIndex or SpeciesType. (References: Section 3.24.4 on page 33.)

\section{Rules for CompartmentReference objects}

multi-22001 $\checkmark \quad$ A CompartmentReference object may have the optional SBML Level 3 Core attributes metaid and sboTerm. No other attributes from the SBML Level 3 Core namespace are permitted on a CompartmentReference object. (References: Section 3.6 on page 12).

multi-22002 $\varangle \quad$ A CompartmentReference object may have the optional SBML Level 3 Core subobjects for notes and annotation. No other elements from the SBML Level 3 Core namespace are permitted on a CompartmentReference object. (References: Section 3.6 on page 12).

multi-22003 $\checkmark \quad$ A CompartmentReference object must have the required attribute multi:compartment, and may have the optional attributes multi:id and multi : name. No other attributes from the Multi namespace are permitted on a CompartmentReference object. (References: Section 3.6 on page 12.)

multi-22004 $\nabla \quad$ The value of the multi : compartment attribute must be the value of an id attribute on an existing Compartment object in the SId namespace of the parent Model. (References: Section 3.6 on page 12.)

multi-22005 $\varangle \quad$ If some or all CompartmentReference objects within a ListOfCompartmentReferences object reference the same Compartment object, those compartmentReferences are required to have its multi : id attribute defined. (References: Section 3.6.1 on page 12.)

multi-22006 $\square \quad$ A compartmentReference cannot reference a compartment that directly or indirectly contains the compartmentReference. (References: Section 3.6.2 on page 12.) 


\section{Acknowledgments}

This work was supported by the Intramural Research Program of the US National Institute of Allergy and Infectious Diseases of the National Institutes of Health.

We sincerely thank all the contributors and their funding agencies. L.P.S., S.M.K, N.R., A.D., F.B. and M.H. were supported by the National Institute of General Medical Sciences (NIGMS, US) grant R01 GM070923. In addition, A.D. was supported by the DZIF (German Center for Infection Research) and by infrastructural funding from the Deutsche Forschungsgemeinschaft (DFG, German Research Foundation), Cluster of Excellence EXC 2124 Controlling Microbes to Fight Infections; and F.B. was supported by the Bundesministerium für Bildung und Forschung (BMBF, DE) grant de.NBI ModSim1, 031L0104A. M.L.B. was supported by NIH (US) grant P41 GM103313 and R24 GM134211. J.F. and J.J.T. were supported by National Institutes of Health (NIH, US) grant P41 GM103712 to the National Center for Multiscale Modeling of Biological Systems (MMBioS). W.S.H was supported by the National Institute of General Medical Sciences (NIGMS, US) grant R01 GM111510. 


\section{References}

Angermann, B. R., Klauschen, F., Garcia, A. D., Prustel, T., Zhang, F., Germain, R. N., and Meier-Schellersheim, M. (2012). Computational modeling of cellular signaling processes embedded into dynamic spatial contexts. Nat Methods, 9(3):283-9.

Danos, V. and Laneve, C. (2004). Formal molecular biology. Theoretical Computer Science, 325(1):69-110.

Faeder, J. R., Blinov, M. L., and Hlavacek, W. S. (2009). Rule-based modeling of biochemical systems with BioNetGen. Methods Mol Biol., 500:113-67.

Feret, J., Danos, V., Krivine, J., Harmer, R., and Fontana, W. (2009). Internal coarse-graining of molecular systems. PNAS, 106:6453-6458.

Hlavacek, W. S., Faeder, J. R., Blinov, M. L., Posner, R. G., Hucka, M., and Fontana, W. (2006). Rules for Modeling Signal-Transduction Systems. Sci. STKE, 344:ref6.

Hucka, M., Bergmann, F., Dräger, A., Hoops, S., Keating, S. M., Le Novère, N., Myers, C. J., Olivier, B. G., Sahle, S., Schaff, J., Smith, L., Waltemath, D., and Wilkinson, D. (2016). The Systems Biology Markup Language (SBML): Language Specification for Level 3 Version 1 Core. Available via the World Wide Web at http://sbml.org/ Documents/Specifications.

Le Novère, N. and Oellrich, A. (2010). Multistate and Multicomponent Species (multi). Available via the World Wide Web at http://sbml.org/Community/Wiki/SBML_Level_3_Proposals/Multistate_and_ Multicomponent_Species_Proposal.

Manes, N. P., Angermann, B. R., Koppenol-Rabb, M., An, E., Sjoelund, V. H., Sun, J., Ishii, M., Germain, R. N., Meier-Schellersheim, M., and Nita-Lazar, A. (2015). Targeted Proteomics-Driven Computational Modeling of Macrophage SIP Chemosensing. Molecular \& Cellular Proteomics, 14(10):2661-81.

Meier-Schellersheim, M., Xu, X., Angermann, B., Kunkel, E. J., Jin, T., and Germain, R. N. (2006). Key role of local regulation in chemosensing revealed by a new molecular interaction-based modeling method. PLoS Comput Biol, 2(7):e82.

Miskov-Zivanov1, N., Turner, M. S., Kane, L. P., Morel, P. A., Faeder, and R., J. (2013). The Duration of T Cell Stimulation Is a Critical Determinant of Cell Fate and Plasticity. Science Signaling, 6(300):ra97.

Zhang, F. (2015). SBML Multi Package: Development Upddate and Discussion. In COMBINE 2015 (http: // co . mbine.org/events/COMBINE_2015/agenda?q=system/files/combine2015-multi_breakout7.pdf).

Zhang, F., Angermann, B. R., and Meier-Schellersheim, M. (2012). Draft for Discussion: SBML Proposals for "Revised Multi", "Simple Spatial" and "Multi-Spatial" Extensions. In COMBINE 2012 (http://co . mbine.org/ events/COMBINE_2012/agenda?q=system/files/2012-08-15-combine-zhang-SBML_prop_v2.pdf).

Zhang, F., Angermann, B. R., and Meier-Schellersheim, M. (2013). The Simmune Modeler visual interface for creating signaling networks based on bi-molecular interactions. Bioinformatics, 29 (9):1229-1230.

Zhang, F. and Meier-Schellersheim, M. (2012). Multistate, Multicomponent and Multicompartment Species Package for SBML Level 3 (Multi), rev 221. Available via the World Wide Web at http: //goo .gl/YGXav4.

Zhang, F. and Meier-Schellersheim, M. (2013a). Multistate, Multicomponent and Multicompartment Species Package for SBML Level 3 (Multi), rev 280. Available via the World Wide Web at http: //goo .gl/2375K.

Zhang, F. and Meier-Schellersheim, M. (2013b). Multistate, Multicomponent and Multicompartment Species Package for SBML Level 3 (SBML-Multi). In HARMONY 2013 (http://goo.gl/DIyEy). 
Zhang, F. and Meier-Schellersheim, M. (2013c). Multistate, Multicomponent and Multicompartment Species Package for SBML Level 3 (SBML-Multi). In COMBINE 2013 (http://co.mbine.org/events/COMBINE_2013/ agenda?q=system/files/sbml_multi_combine_2013\%20presentation_FengkaiZhang.pdf).

Zhang, F. and Meier-Schellersheim, M. (2014). SBML Multi Package (Breakout session). In COMBINE 2014 (http: //co.mbine.org/events/COMBINE_2014/agenda?q=system/files/Fengkai_Multi_breakout_v6_(1.pdf). 